Supporting Information for

\title{
Adipogenic activity of chemicals used in plastic
}

\section{consumer products}

Johannes Völker ${ }^{1}$, Felicity Ashcroft ${ }^{1}$, Asa Vedøy ${ }^{1}$, Lisa Zimmermann ${ }^{2}$ and Martin Wagner ${ }^{{ }^{*}}$

${ }^{1}$ Department of Biology, Norwegian University of Science and Technology (NTNU), 7491

Trondheim, Norway

2 Department of Aquatic Ecotoxicology, Goethe University Frankfurt am Main, 60438

Frankfurt am Main, Germany

*corresponding author: Martin Wagner, Email: martin.wagner@ntnu.no

Number of pages: 53

Number of figures: 24

Number of tables: 5 


\section{Supporting Materials and Methods}

\section{Plastic extraction}

3 We used the original samples stored in glassware, except for PLA 3. As there was not enough

4 material available for the original PLA 3 sample and we were not able to obtain more of the

5 same product, we replaced the sample with a PLA coffee lid sample. To avoid sample 6 contamination, glass or polytetrafluorethylene consumables were used for the sample

7 extraction and all material was rinsed twice with acetone and annealed at $200{ }^{\circ} \mathrm{C}$ for $\geq 3 \mathrm{~h}$. The

8 samples were cut into $0.5-0.8 \times 2 \mathrm{~cm}$ pieces. Foamy products were cut to a thickness of 0.5

$9 \mathrm{~cm}$. We weighed three grams each into 1 or 2 transparent glass vials depending on the sample

10 volume, added $20 \mathrm{~mL}$ methanol ( $\geq 99.9 \%$, Sigma Aldrich), and extracted the samples by

11 sonication in an ultrasonic bath for $1 \mathrm{~h}$ at room temperature. We then transferred the methanol

12 into clean glass vials, added $200 \mu \mathrm{L}$ dimethyl sulfoxide ( $\geq 99.5 \%$, Sigma Aldrich) as a keeper

13 and evaporated the samples under a gentle stream of nitrogen to a final volume of $200 \mu \mathrm{L}$.

14 Further, we treated three procedural blanks (PB 1-3) not containing any sample identically to control for contamination and stored the final extracts at $-20{ }^{\circ} \mathrm{C}$ prior to analysis.

\section{Cell culture conditions}

17 3T3-L1 cells were cultured in preadipocyte medium (PAM: DMEM-high supplemented with 10\% bovine calf serum and 1\% penicillin/streptomycin). Culturing 3T3-L1 cells over multiple passages can cause a decline in differentiation efficiency due to the loss of contact inhibition. ${ }^{1}$

20 Thus, cryo-cultures of passage 9 were thawed, subcultured once upon reaching 60-80\% confluency, and sub-confluent cells of passage 10 were used for all experiments to ensure comparability and preserve differentiation capability. CALUX cells were maintained in growth 
penicillin/streptomycin, and $0.2 \mathrm{mg} \mathrm{mL}^{-1} \mathrm{G} 418$ and non-essential amino acids) and subcultured twice weekly and used until passage 20 .

\section{Optimization of the adipogenesis assay}

27 We conducted optimization experiments to identify a suitable concentration of dexamethasone (DEX) to initiate adipocyte differentiation. Moreover, we applied high-content fluorescence imaging combined with an automated image processing in addition to the fluorescence readout well ${ }^{-1}$ at the end of the experiment and compared both methods with regards to sensitivity.

Following the growth arrest window, the medium was replaced with $200 \mu \mathrm{L}$ differentiation medium well ${ }^{-1}$ (DM: DMEM-high supplemented with 10\% FBS, 1\% penicillin/streptomycin, $20 \mathrm{mM}$ HEPES, $1 \mu \mathrm{g} \mathrm{mL} \mathrm{m}^{-1}$ insulin and $0.5 \mathrm{mM}$ 3-isobutyl-1-methylxanthine (IBMX)) containing either none or six concentrations of dexamethasone $(6.25-250 \mathrm{nM})$ and eight concentrations of the reference compound rosiglitazone $(300 \mathrm{pM}-1 \mu \mathrm{M})$. After the $48-\mathrm{h}$ differentiation window, we replaced the medium with $200 \mu \mathrm{L}$ adipocyte maintenance medium well ${ }^{-1}$ (DM without IBMX and DEX) containing the eight rosiglitazone concentrations and changed the medium every other day during the maintenance period.

Counting nuclei based on NucBlue staining using the imaging approach was more sensitive than the standard fluorescence readout for detecting the proliferative effect of rosiglitazone (Figure S3 A) with an $\mathrm{EC}_{50}$ of 16.3 and $41.2 \mathrm{nM}$ for nuclei counts and fluorescence readout, respectively. In contrast, quantification of adipogenesis had comparable sensitivity (Figure S3 B) with an $\mathrm{EC}_{50}$ of 10.6 and $11.4 \mathrm{nM}$ for lipid droplet intensity and total NileRed fluorescence readout, respectively. However, the dynamic range of the assay was greatly enhanced using the imaging approach with an 11.7-fold increase in the lipid droplet count at the highest rosiglitazone concentration versus 4.69 -fold increase in the fluorescence readout.

47 In addition, the imaging-based approach provides more information including the 
characterization of the differentiation stage of cells in the population, and single-cell measurements to quantify the size of adipocytes and triglyceride accumulation (e.g., number and size of lipid droplets). Thus, high-content imaging with automated image processing can greatly extend our capabilities fir screening of MDCs in vitro.

52 Glucocorticoids in the differentiation medium are essential to prime the preadipocytes for adipogenesis and the differentiation success is variable and weak when DEX is absent (Figure S24). In contrast, an excess of DEX (>25 nM) results in a significant stimulation of adipogenesis without an additional inducer and, thus, reduces the capability of the assay to detect adipogenic responses (Figure S3 C). This is in line with a previous study reporting up to $40 \%$ of adipocytes in the vehicle controls using $250 \mathrm{nM}$ DEX in the differentiation medium. ${ }^{2}$ Accordingly, the use of DEX concentrations varying from $0^{3-5}$ up to $1 \mu \mathrm{M}^{1,2,6,7}$ might contribute to the poor reproducibility and comparability of 3 T3-L1 studies. ${ }^{8}$ Based on our experiments, we recommend using a rather low DEX concentration of $6.25 \mathrm{nM}$ which was sufficient to initiate adipocyte differentiation without increasing the assay baseline (Figure S3 D).

\section{Fixation and staining}

64 After $11 \mathrm{~d}$, the medium was removed, and cells were rinsed with PBS and fixed with 2\% paraformaldehyde for $10 \mathrm{~min}$ on ice. The fixative was removed, and cells were rinsed twice with PBS and stored at $4{ }^{\circ} \mathrm{C}$ prior to staining. Cells were co-stained with $100 \mu \mathrm{L}$ NileRed solution well ${ }^{-1}$ (19.5 mL PBS $+500 \mu$ L AdipoRed (N3013, Lonza) and 1 drop mL ${ }^{-1}$ NucBlue (R37605, Thermo, Hoechst 3342 staining)). Plates were incubated for $40 \mathrm{~min}$ in the dark at room temperature. Stained cells were washed twice with PBS and stored at $4{ }^{\circ} \mathrm{C}$ prior to analysis. 
71 Fluorescence per well was measured using a Cytation 5 Cell Imaging Multimode reader

72 (BioTek with excitation at $485 \mathrm{~nm}$ and emission at $572 \mathrm{~nm}$ for NileRed, and excitation at 360 $\mathrm{nm}$ and emission at $460 \mathrm{~nm}$ for NucBlue). Imaging was carried out on the same instrument using a 10× Plan Fluorite objective (WD10, NA 0.3). Image-based autofocusing of NucBlue fluorescence was used to select the image plane, and three images per field were captured (Brightfield, NucBlue and NileRed). A 365 LED with DAPI filter cube (Ex 377/50, Em 447/60) was used to detect the NucBlue staining, and a 523 LED with RFP filter cube (Ex 531/40, Em 593/40) for NileRed. Nine fields were captured per well.

\section{Cell profiler analysis}

80 For the adipogenesis assay, NucBlue and NileRed staining imaged at x10 magnification were 81 analyzed using the following protocol to generate the assay measurements described.

82 1. Cell identification: nuclei (primary objects) were identified using an Otsu thresholding method based on NucBlue staining and used as the seed objects to identify cells (secondary objects). NileRed images were smoothed by gaussian filtration and used to guide the propagation algorithm for secondary object identification with a minimal threshold factor to 86 limit the foreground.

2. Lipid droplet identification (adapted from Adomshick et al. $)^{9}$ : lipid droplets were identified using a minimum cross entropy thresholding method applied to the NileRed images followed by a filtration step based on the mean intensity per droplet.

3. Image-based measurements: the number of cells and the number of lipid droplets were

91 counted in each image. We additionally measured the total area occupied by lipid droplets, and

92 the intensity of the Nile Red staining in this region. 
93 4. Single cell analysis: to measure the lipid content per cell, lipid droplets were assigned to a

94 given parent and merged such that the total area occupied by lipid, and the average intensity of

95 the NileRed staining in this region could be calculated.

96 5. Data processing: filtration steps were subsequently applied to identify adipocytes (any cell

97 containing at least one lipid droplet), and mature adipocytes (a cell having a lipid droplet area

$98 \geq 1000$ pixels, equivalent to $\geq 8$ average size lipid droplets.)

99 For the nuclear counts (reporter gene assays), NucBlue staining imaged at $\mathrm{x} 4$ magnification

100 was analyzed using the following protocol to quantify the number of nuclei in a given field.

101 Nuclear counts were used for normalization and calculation of cytotoxicity.

102 1. Image correction: we applied the background method, with smoothing based on a gaussian

103 filter to calculate an illumination function which was applied to NucBlue images to correct for

104 the uneven illumination resulting from imaging the 384 well plates at $\times 4$ magnification.

105 2. Identification of nuclei: nuclei were identified using an Otsu thresholding method based on 106 the corrected images and we filtered the resulting objects based on their shape (form factor) to

107 obtain final nuclear counts.

108 The cell profiler pipelines can be accessed under DOI 10.5281/zenodo.5513372

\section{Reporter gene assays}

110 We performed the CALUX reporter gene assays (U2OS cell lines) in white clear polystyrene

111 CellStar 384-well plates (781098, Greiner Bio-One). Trypsinized cells were resuspended in

112 assay medium (DMEM/F-12 without phenol red supplemented with 5\% charcoal-stripped

113 FBS, 1\% penicillin/streptomycin, non-essential amino acids). 3000 cells well $^{-1}$ were seeded in

$11425 \mu \mathrm{L}$, and plates were incubated at $37^{\circ} \mathrm{C}$ and $5 \% \mathrm{CO}_{2}$. Samples and reference compounds 
115 were prepared in assay medium (2-fold higher than the final assay concentration) in six

116 concentrations per sample serially diluted 1:2 or eight concentrations of the reference

117 compound (rosiglitazone for PPAR $\gamma$ and dexamethasone for GR, Figure S4). After $24 \mathrm{~h}$ of

118 incubation, $25 \mu \mathrm{L}$ sample was added to the $25 \mu \mathrm{L}$ assay medium well-1 ${ }^{-1}$-fold), resulting in

119 final sample concentrations of $0.05-1.5 \mathrm{mg}$ plastic well ${ }^{-1}$ (equivalent to $0.09-30 \mathrm{mg}$ plastic $\mathrm{mL}^{-}$

$\left.12{ }^{1}{ }^{1}\right)$. After $23 \mathrm{~h}$ of exposure, the medium was replaced with $25 \mu \mathrm{L}$ NucBlue staining solution

121 well $^{-1}$ (1 drop NucBlue per mL PBS, Thermo Fisher Scientific) and incubated for 30 min in the

122 dark at room temperature.

123 Imaging was performed on the Cytation 5 Cell Imaging Multimode reader (BioTek) with a 4×

124 Plan Fluorite objective (WD 17 NA 0.13) using a 365 LED with DAPI filter cube (Ex 377/50,

125 Em 447/60) to detect NucBlue staining. A single field was captured per well. Following the

126 imaging, a white sticker was placed on the transparent bottom of the plates, and the staining

127 solution was replaced with $20 \mu \mathrm{L}$ cell lysis buffer ( $25 \mathrm{mM}$ pH 7.8 TRIS, $2 \mathrm{mM}$ DDT, $2 \mathrm{mM}$

128 CDTA, 10\% glycerol, and 1\% Tritron-X100), and cells were lysed by linear shaking for 3 min.

129 Luminescence was measured (Cytation 5) for one second after injection of $30 \mu \mathrm{L}$ illuminate

$130 \operatorname{mix}\left(20 \mathrm{mM}\right.$ Tricine, $1.07 \mathrm{mM} \mathrm{C}_{4} \mathrm{H}_{2} \mathrm{Mg}_{5} \mathrm{O}_{14}, 2.67 \mathrm{mM} \mathrm{MgSO}_{4} \times 7 \mathrm{H}_{2} 0,0.1 \mathrm{mM}$ EDTA,

131 1.5 mM DDT, $539 \mu \mathrm{M}$ D-Luciferine, $5.49 \mathrm{mM} \mathrm{ATP)} \mathrm{followed} \mathrm{by} \mathrm{quenching} \mathrm{of} \mathrm{the} \mathrm{reaction}$

132 with $30 \mu \mathrm{L} 0.1 \mathrm{M} \mathrm{NaOH}$.

\section{Analysis of bioassay data}

134 We used GraphPad Prism 9 (GraphPad Software, San Diego, CA) for non-linear regressions and statistical analysis. To express cytotoxicity, we normalized the nuclei count to the vehicle controls ( $0 \%$ cytotoxicity) and a value of zero (100\% cytotoxicity). We used $20 \%$ as

137 cytotoxicity threshold. When the value of an individual image was $>20 \%$, all channels of that 138 image were excluded from further analysis (cytotoxic or out of focus). If the mean value of a 
139 replicate exceeded $20 \%$, the replicate was excluded. When more than one replicate per

140 concentration exceeded the threshold, the concentration was defined as cytotoxic. Fluorescence

141 and luminescence readouts were corrected for background (wells without cells). Percentage

142 increase (proliferative effects) or fold induction over the corresponding vehicle control were

143 calculated for each endpoint of the adipogenesis assay to compare both methods in the 144 optimization experiments. To express agonistic activity in the reporter gene assays,

145 luminescence data were normalized to the maximal assay response (100\% activity: upper

146 plateau of the dose-response relationship) of the corresponding reference compound and the

147 mean value of the vehicle control ( $0 \%$ activity). The limit of detection (LOD) of each endpoint

148 and experiment was calculated as three times the standard deviation (SD) of pooled controls

149 (equivalent to a $\mathrm{z}$-score of 3). For samples to be considered adipogenic, these had to trigger

150 effects $\geq$ LOD in at least two endpoints in the adipogenesis assays. For samples to be considered

151 agonists of PPAR $\gamma$ or GR, the reporter gene activity had to be $\geq$ LOD. Dose-response

152 relationships for all investigated endpoints were calculated using a four-parameter logistic

153 function constrained to the lower plateau ( $0 \%$ activity). The respective plastic equivalents 154 inducing 10 or $20 \%$ effect (effect concentration, $\mathrm{EC}_{10}$, or $\mathrm{EC}_{20}$ ) were interpolated from the dose-response curves.

\section{Nontarget chemical analysis}

157 We analyzed all samples, except PLA 3, using ultra-high performance liquid chromatography 158 coupled to a quadrupole time of flight spectrometer (LC-QTOF-MS/MS) with an Acquity

159 UPLC Waters liquid chromatography system coupled to a SYNAPT G2-S mass spectrometer

160 (both Waters Norge, Oslo, Norway). The analytical method has been described previously. ${ }^{10,}$

$161{ }^{11}$ In brief, we injected $2 \mu \mathrm{L}$ sample, equivalent to the chemicals extracted from $1.5 \mathrm{mg}$ plastic,

162 and performed the chromatographic separation on an Acquity UPLC BEH C18 column 163 equipped with a C18 guard column (both from Waters). The mass spectrometer equipped with 
an electron-spray ionization source was operated in positive ionization mode with a mass range of 50-1200 Da at a resolution of 20,000. MS data were recorded from 2-35.5 min with a datadependent acquisition (triggered when the threshold of an individual ion intensity exceeded 25,000 counts, maximum 15 precursor ions per survey scan) using a collision energy ramp (8-

$16835 \mathrm{eV}$ in the low mass region and $30-70 \mathrm{eV}$ in the high mass region). After every $7^{\text {th }}-8^{\text {th }}$ sample, we analyzed a solvent blank (mobile phase, methanol or DMSO, $\mathrm{n}=15$ ) and a quality control sample (containing an aliquot of each sample). Two PBs from the extraction were also analyzed. The raw mass spectral data can be accessed under DOI 10.5281/zenodo.4781257 (published after publication).

\section{Chemical data analysis and compound identification}

174 We imported the data for the 15 blanks, two PBs, six quality controls, and 33 samples to 175 Progenesis QI (version 3.0, Nonlinear Dynamics) and corrected for the lock mass of leucine enkephalin. We automatically aligned the retention times of all blanks and samples using the quality controls. Peak picking was performed on the samples using common adducts $(\mathrm{M}+\mathrm{H}$, $\mathrm{M}+2 \mathrm{H}, \mathrm{M}+\mathrm{H}-\mathrm{H} 2 \mathrm{O}, \mathrm{M}+\mathrm{H}-2 \mathrm{H}_{2} \mathrm{O}, 2 \mathrm{M}+\mathrm{H}, \mathrm{M}+\mathrm{Na}, \mathrm{M}+2 \mathrm{Na}, \mathrm{M}+\mathrm{H}+\mathrm{Na}, \mathrm{M}+2 \mathrm{H}+\mathrm{Na}, \mathrm{M}+2 \mathrm{Na}+\mathrm{H}$, $\mathrm{M}+2 \mathrm{Na}-\mathrm{H})$, an automatic sensitivity, a minimum peak width of $0.02 \mathrm{~min}$ and a fragment sensitivity of $0.2 \%$ of the base peak.

We generated a list of chemical features which had MS/MS data and performed the further data analysis as described before. ${ }^{11}$ Basically, we filtered for features that were not detected in the solvent and PBs or present in the samples with an at least 10-fold higher raw abundance than the maximum abundance of that feature in any of the blanks.

To tentatively identify the remaining features, we compared their mass spectra with the

186 empirical spectra in MassBank (14,788 compounds, release version 2021.03, 187 https://github.com/MassBank/MassBank-data/releases/tag/2021.03) and with three databases 
covering chemicals present in plastic packaging (2680 compounds), registered under the

189 REACH regulation in 2020 (7092 compounds) and (pre)registered under REACH in 2017

190 (65,738 compounds) using the Metascope algorithm in Progenesis QI with a precursor

191 tolerance of $5 \mathrm{ppm}$ and a fragment tolerance of $10 \mathrm{ppm}$. The compounds in the latter three

192 databases were in silico fragmented using the Metascope algorithm. The resulting identification

193 was accepted if the match score was $>40$. When a feature had multiple identifications with a

194 score $>40$, the first hit with the highest score was accepted. The identification corresponds to confidence level 3 according to Schymanski et al. ${ }^{12}$

\section{Comparison with chemicals known to induce adipogenesis}

197 We built a list of know adipogenic chemicals by searching Web of Science (Core Collection)

198 for studies investigating chemicals in the adipogenesis assay using the following search strings:

199 “(3T3L1 OR 3T3-L1) AND toxic* AND chemical*” (58 hits) as well as "(3T3L1 OR 3T3-L1)

200 AND (obesogen* OR metabolic disruptor*) AND in vitro" (241 hits). The search was conducted on March 22, 2021. We removed duplicates and reviews and screened the remaining 254 full text articles for studies that investigated the adipogenic activity of chemicals in 3T3-

L1 adipocytes. We decided not to perform a quality assessment to keep the list broad and included from 47 suitable studies all chemicals which were reported to be adipogenic. We further complemented the list with the chemicals reviewed by Amato et al. ${ }^{13}$ and ended up with a list of 120 adipogenic chemicals (Table S2). For comparison with our results, we added the associated PubChem CIDs.

To cross-reference this list with the compounds we tentatively identified in plastics, we built a

209 joint compound list based on our previous GC-QTOF-MS/MS analysis ${ }^{14}$ and the present LC-

210 QTOF-MS/MS analysis. For the former, we translated the available CAS numbers of all tentatively identified compounds to SMILES using the US EPA's CompTox Dashboard 
212 (https://comptox.epa.gov/dashboard) and then translated the SMILES to PubChem CIDs using

213 the PubChem Identifier Exchange Service (https://pubchem.ncbi.nlm.nih.gov/idexchange). If

214 CAS numbers were invalid or unavailable, we searched the compound name in PubChem and

215 manually annotated the CID. For the LC-QTOF-MS/MS data, we used the PubChem CIDs

216 provided by Progenesis QI or manually annotated the compound names provided by

217 MassBank. The combined list from the GC- and LC-QFOT/MS/MS data contained 803 unique

218 chemicals with CIDs (Table S3 and Excel Table S1). To determine whether some of these

219 compounds are MDCs, we cross-referenced both CID lists (Table 1). 

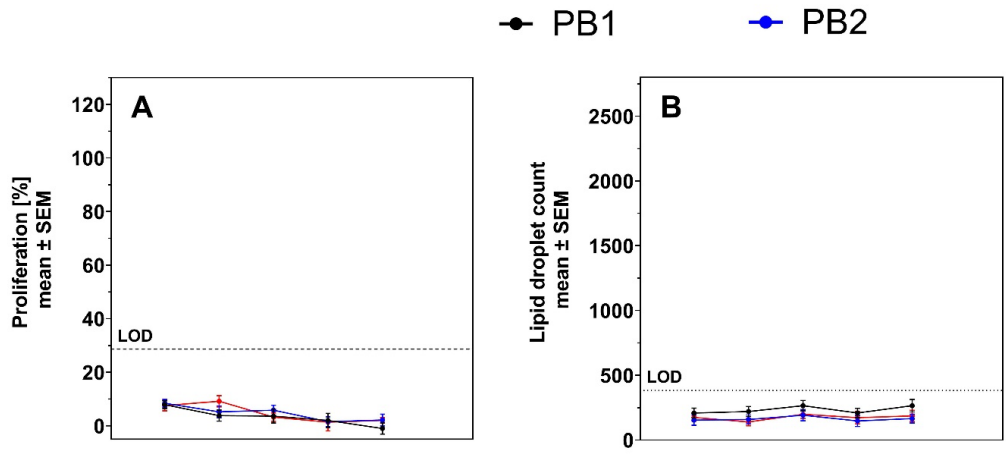

PB3
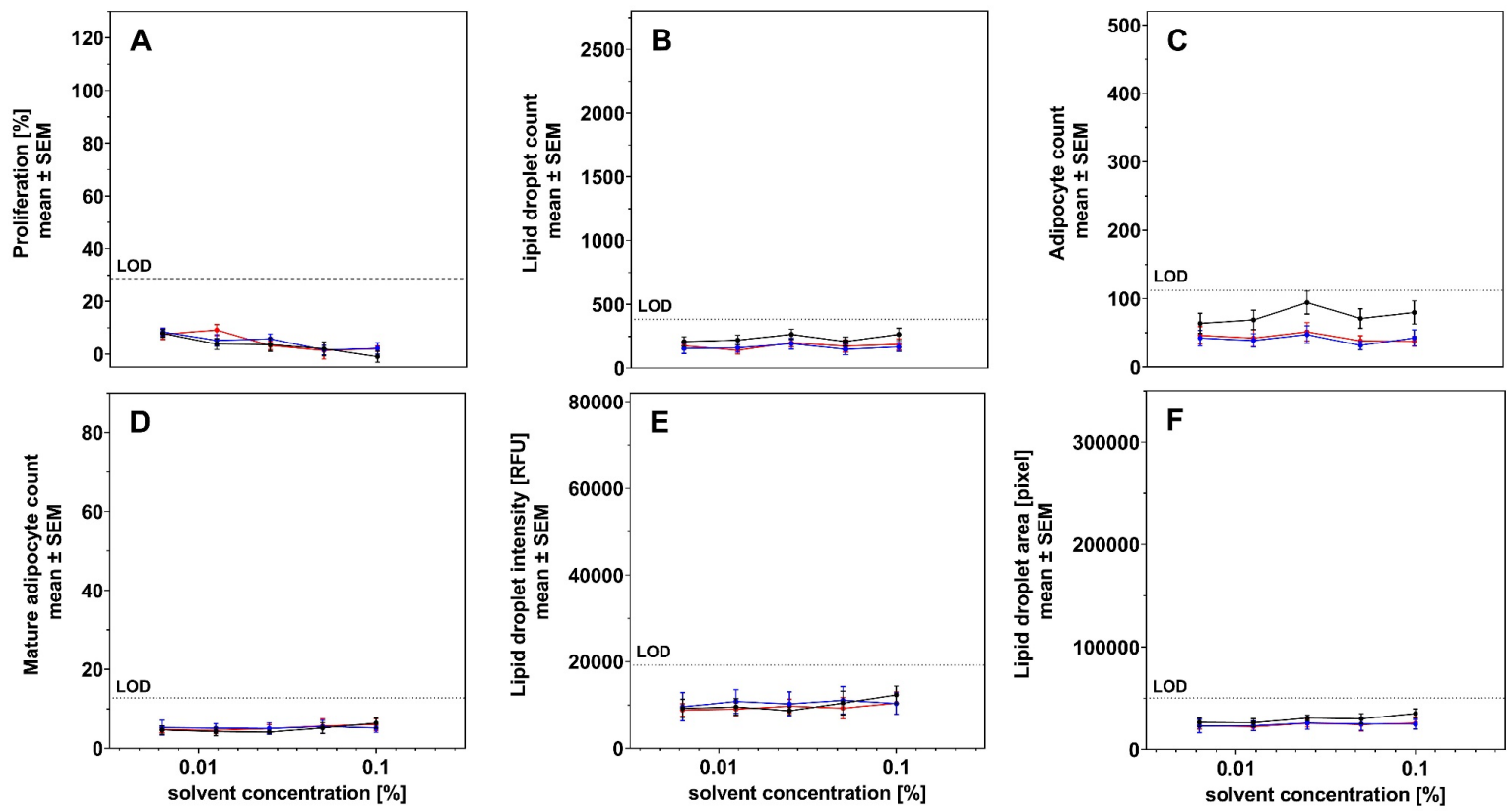

Figure S1. Dose-response relationships for the adipogenesis assay endpoints of the three procedure blanks (PB 1-3). (A) proliferation normalized on the mean of the vehicle control, (B) lipid droplet count per field, (C) adipocyte count per field, (D) mature adipocyte count per field, (E) total intensity of the NileRed staining within the lipid droplet mask per field and (F) total area occupied by lipid droplets per field. Twelve replicates per concentration $(n=12)$. $\mathrm{LOD}=$ limit of detection, RFU = relative fluorescence unit.
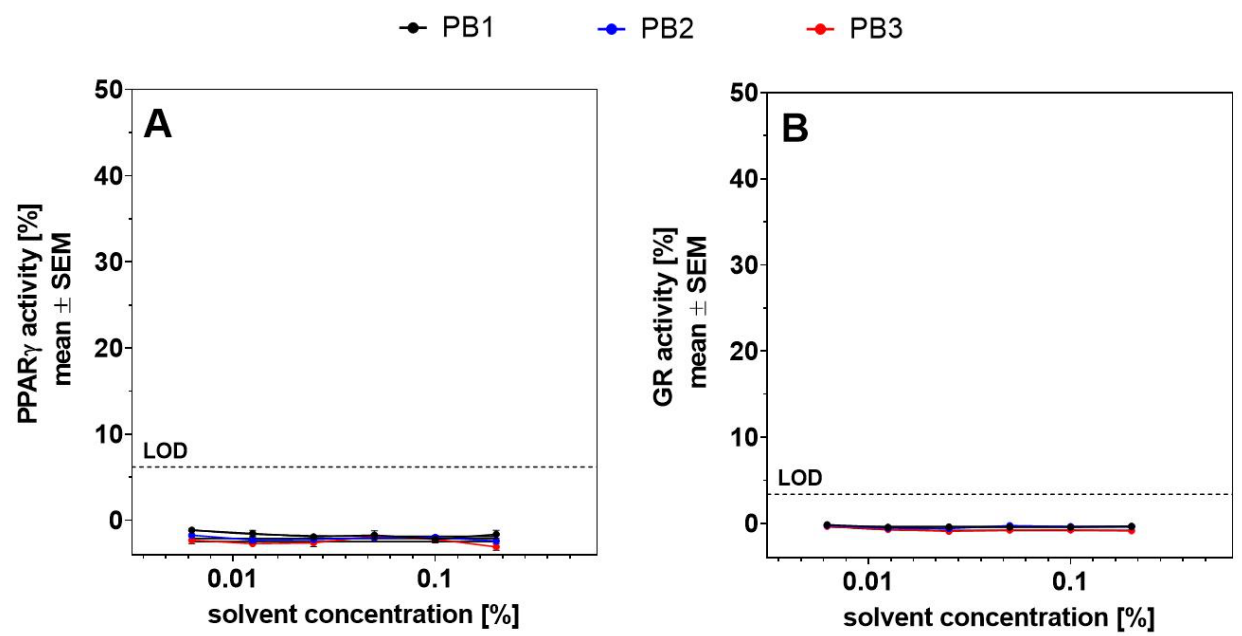

228 Figure S2. Dose-response relationships for (A) PPAR $\gamma$ and (B) GR activity of the three procedure blanks (PB 1-3). Twelve or more replicates per concentration $(n \geq 12)$. PPAR $\gamma=$

230 peroxisome proliferator receptor gamma, GR $=$ glucocorticoid receptor, LOD = limit of 231 detection. 

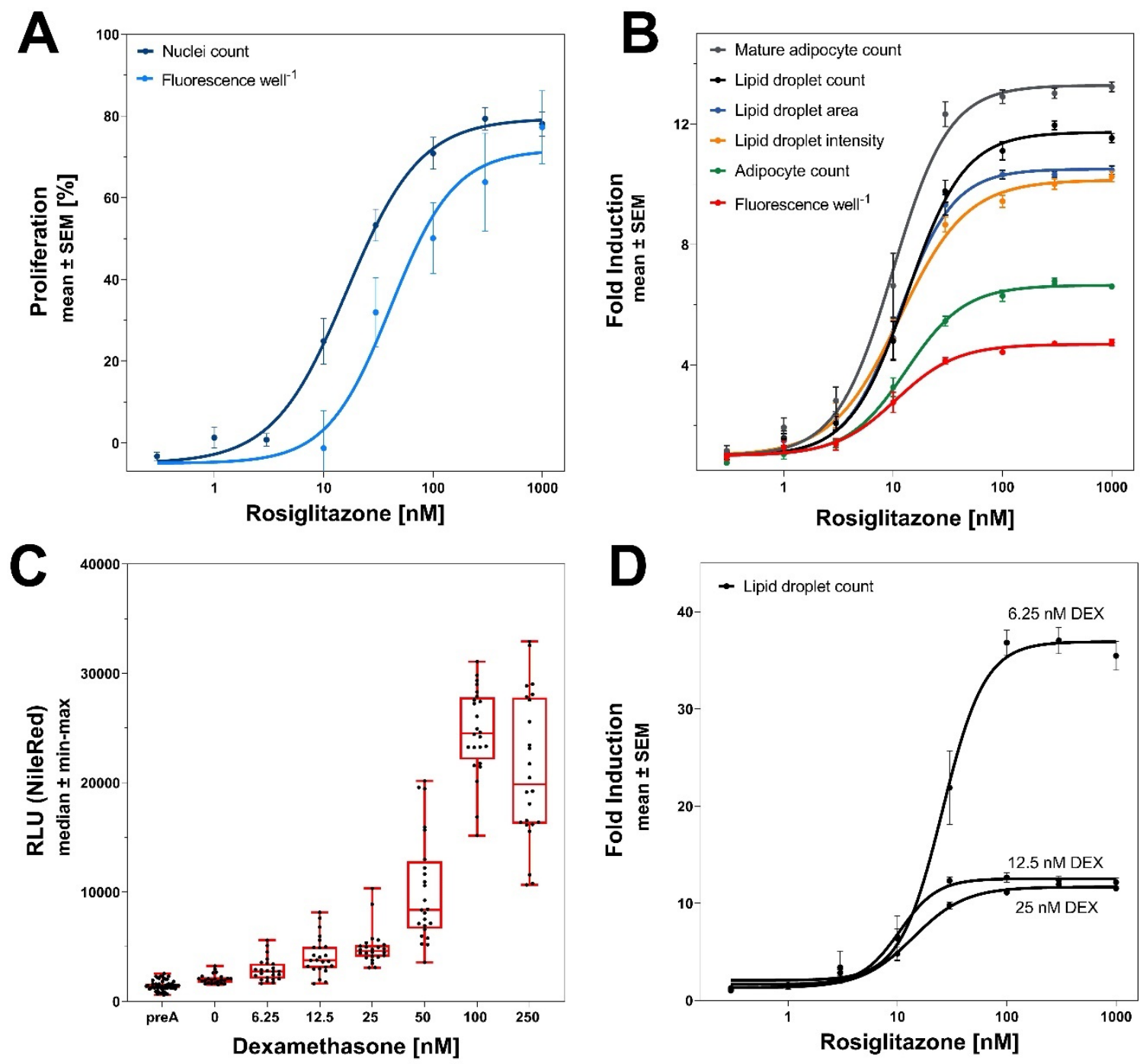

233 Figure S3. Optimization of the adipogenesis assay. (A) Proliferative effects induced by 234 rosiglitazone analyzed by imaging (DAPI filter) and total fluorescence readout (NucBlue) with $23525 \mathrm{nM}$ dexamethasone (DEX) in the differentiation medium (DM). (B) Multiple adipogenic 236 endpoints analyzed by imaging (RFP filter) compared to total fluorescence readout (NileRed) 237 with $25 \mathrm{nM}$ DEX in DM. (C) Total fluorescence readout (NileRed) for the preadipocyte control 238 (preA, undifferentiated) and the differentiated vehicle controls with increasing dexamethasone 239 concentrations in DM. (D) Lipid droplet count induction by rosiglitazone with 6.25, 12.5, $24025 \mathrm{nM}$ DEX in DM. 

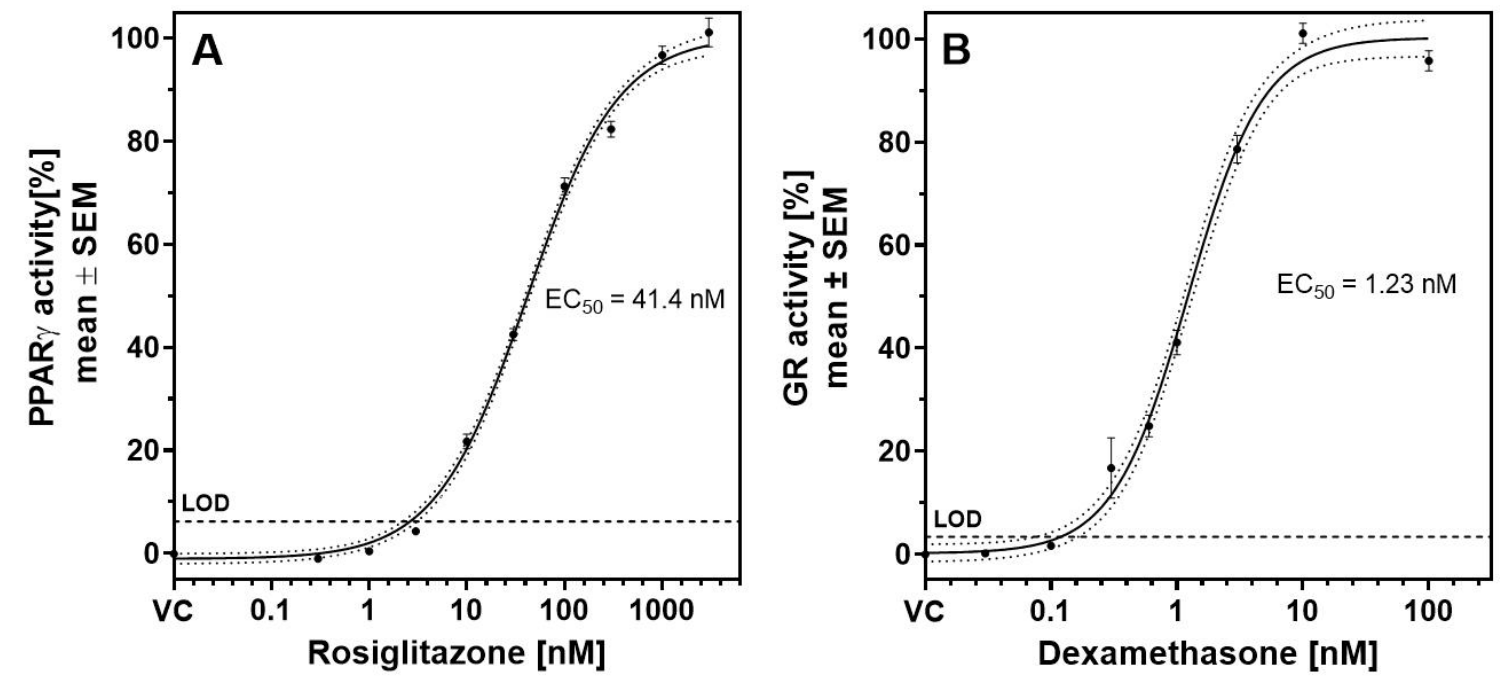

242 Figure S4. Dose-response relationships for (A) PPAR $\gamma$ and (B) GR activity of the

243 reference compound rosiglitazone and dexamethasone. 48 or more replicates per 244 concentration $(n \geq 48)$. PPAR $\gamma=$ peroxisome proliferator receptor gamma, $\mathrm{GR}=$ glucocorticoid 245 receptor, $\mathrm{LOD}=$ limit of detection, $\mathrm{VC}=$ vehicle control.
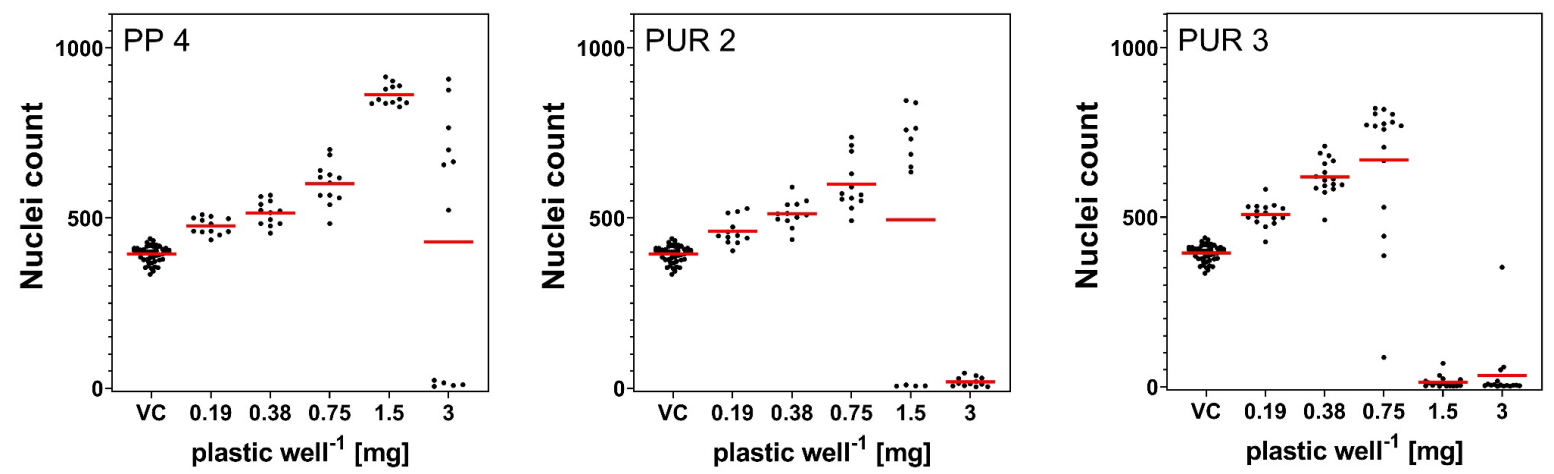

247 Figure S5. Nuclei count of the cytotoxic plastic extracts (PP 4, PUR 2, PUR 3) in the

248 adipogenesis assay. Data is presented as mean count per field (red lines) from three to four 249 independent experiments performed with four replicates each (dots, $n \geq 12$ ). VC $=$ vehicle 250 control. 

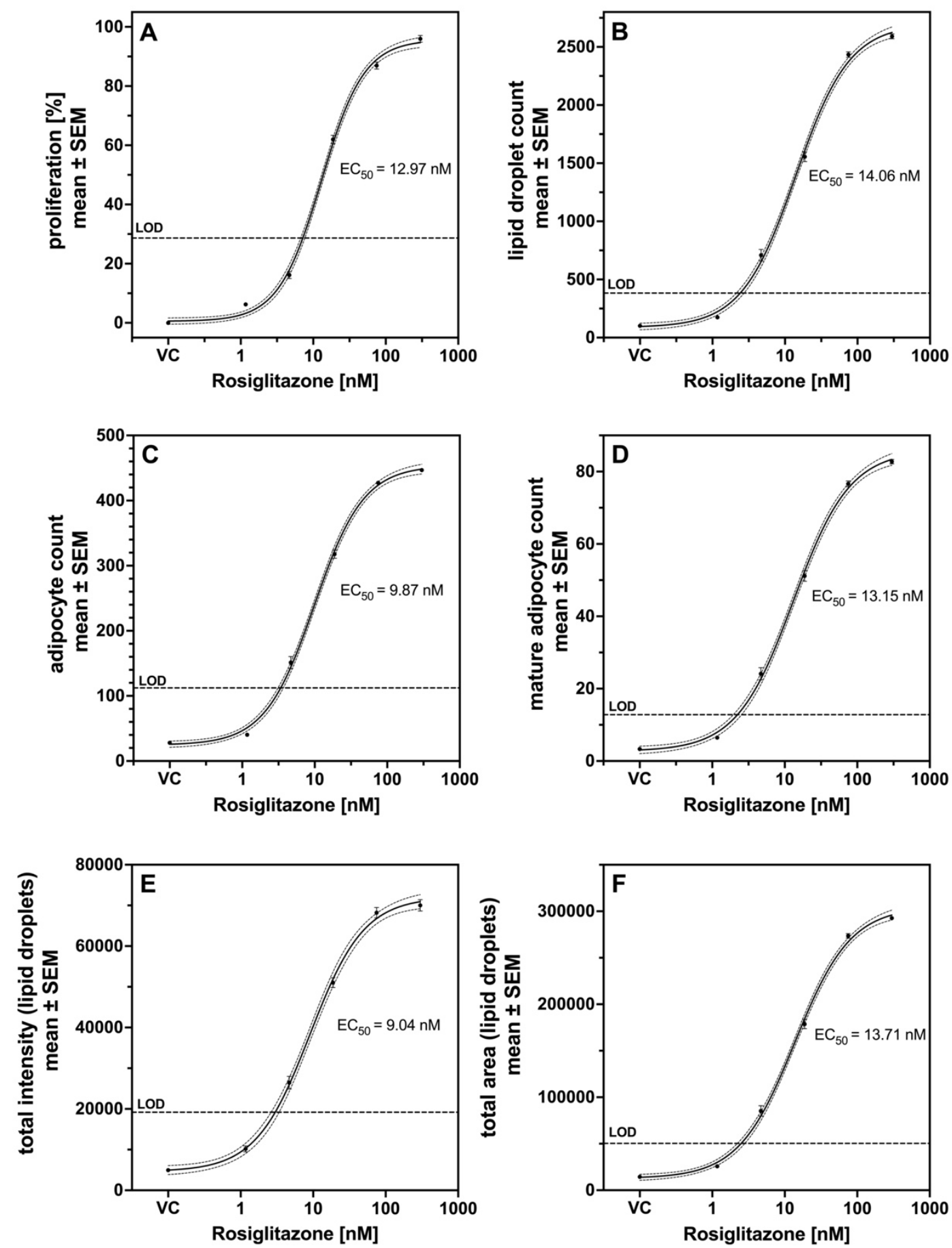

252 Figure S6. Dose-response relationship for the reference compound rosiglitazone in the 253 adipogenesis assay with $6.25 \mathbf{n M}$ dexamethasone in the differentiation medium.

254 (A) proliferation normalized on the mean of the vehicle control, (B) lipid droplet count per 255 field, (C) adipocyte count per field, (D) mature adipocyte count per field, (E) total intensity of 256 the NileRed staining within the lipid droplet mask per field and (F) total area occupied by lipid 257 droplets per field. 160 or more replicates per concentration $(\mathrm{n} \geq 160)$. VC $=$ vehicle control, $258 \mathrm{LOD}=$ limit of detection, RFU = relative fluorescence unit. 

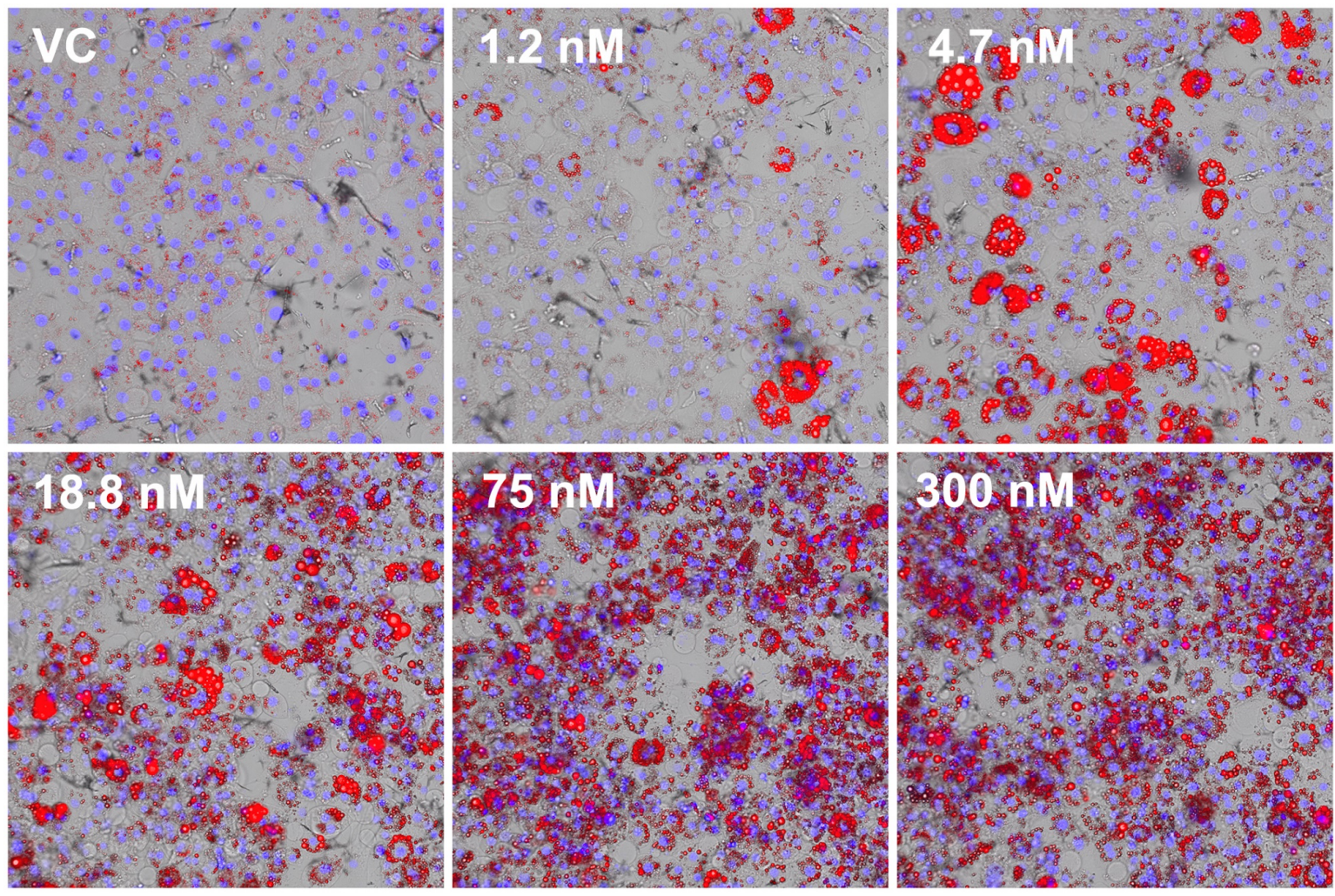

260 Figure S7. Dose-dependent induction of adipogenesis in 3T3-L1 cells exposed to the

261 reference compound rosiglitazone. Merged brightfield and fluorescence images. Nuclei are 262 stained with NucBlue (blue) and triglycerides with NileRed (red). VC = vehicle control. Raw 263 pictures were processed in the same manner for visualization. 

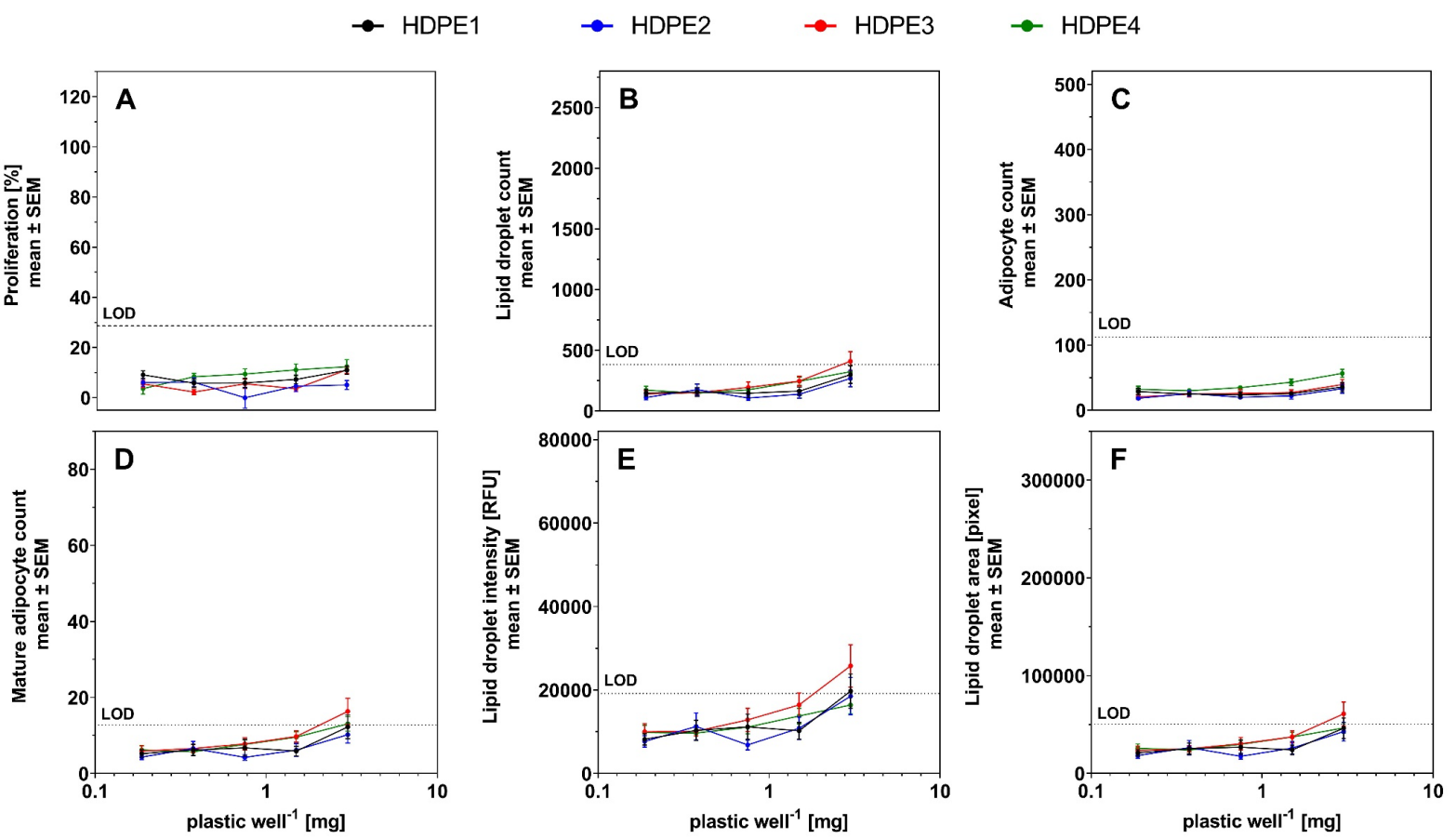

265 Figure S8. Dose-response relationship for the HDPE plastic extracts (HDPE 1-4) in the

266 adipogenesis assay. (A) proliferation normalized on the mean of the vehicle control, (B) lipid 267 droplet count per field, (C) adipocyte count per field, (D) mature adipocyte count per field, (E) 268 total intensity of the NileRed staining within the lipid droplet mask per field and (F) total area 269 occupied by lipid droplets per field. Twelve or more replicates per concentration ( $\mathrm{n} \geq 12)$. LOD $270=$ limit of detection, $\mathrm{RFU}=$ relative fluorescence unit. 

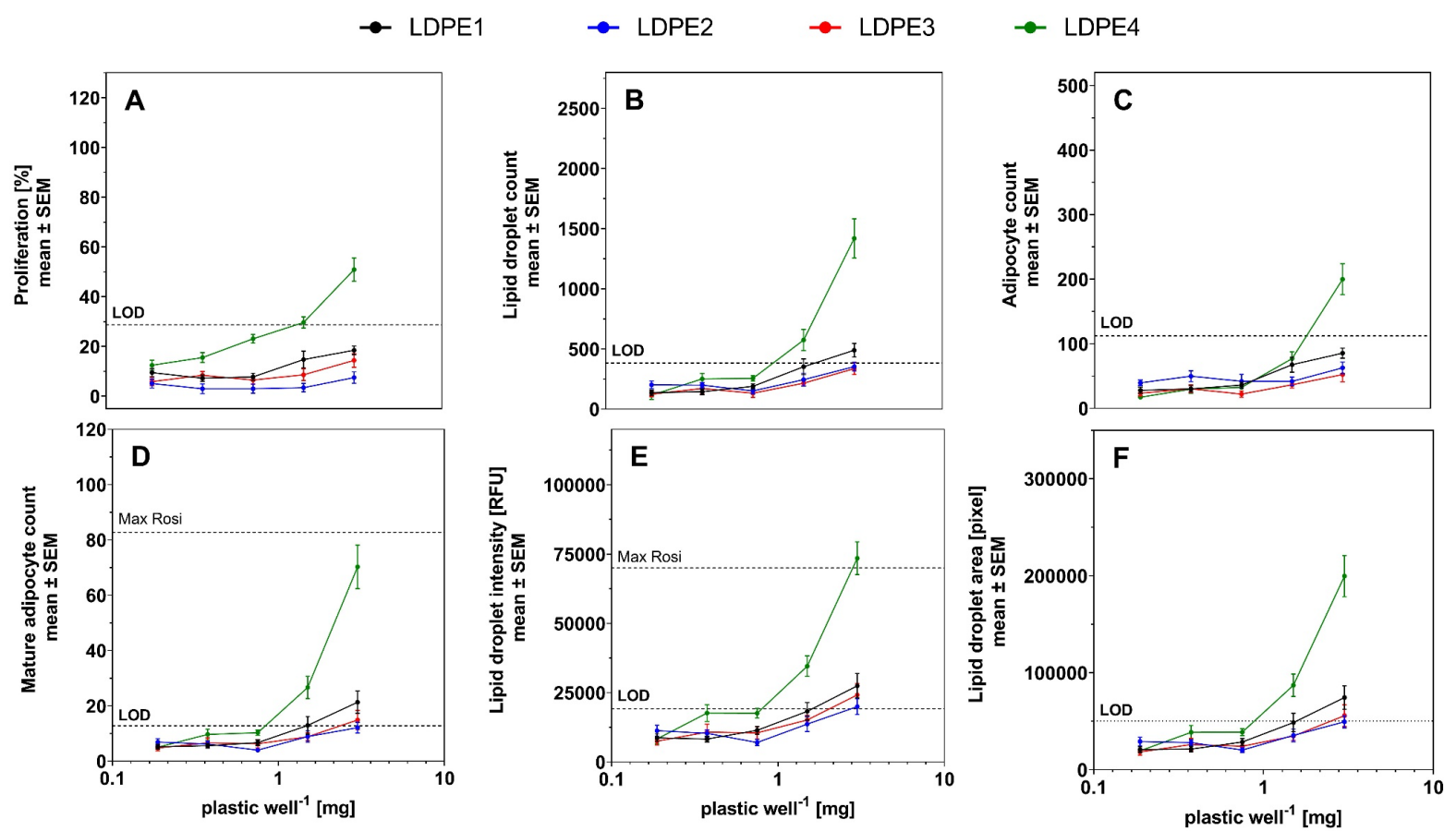

272 Figure S9. Dose-response relationship for the LDPE plastic extracts (LDPE 1-4) in the

273 adipogenesis assay. (A) proliferation normalized on the mean of the vehicle control, (B) lipid 274 droplet count per field, (C) adipocyte count per field, (D) mature adipocyte count per field, (E) 275 total intensity of the NileRed staining within the lipid droplet mask per field and (F) total area 276 occupied by lipid droplets per field. Twelve or more replicates per concentration $(n \geq 12)$. LOD $277=$ limit of detection, Max Rosi = rosiglitazone maximal response, RFU = relative fluorescence 278 unit. 
280

281

282

283

284
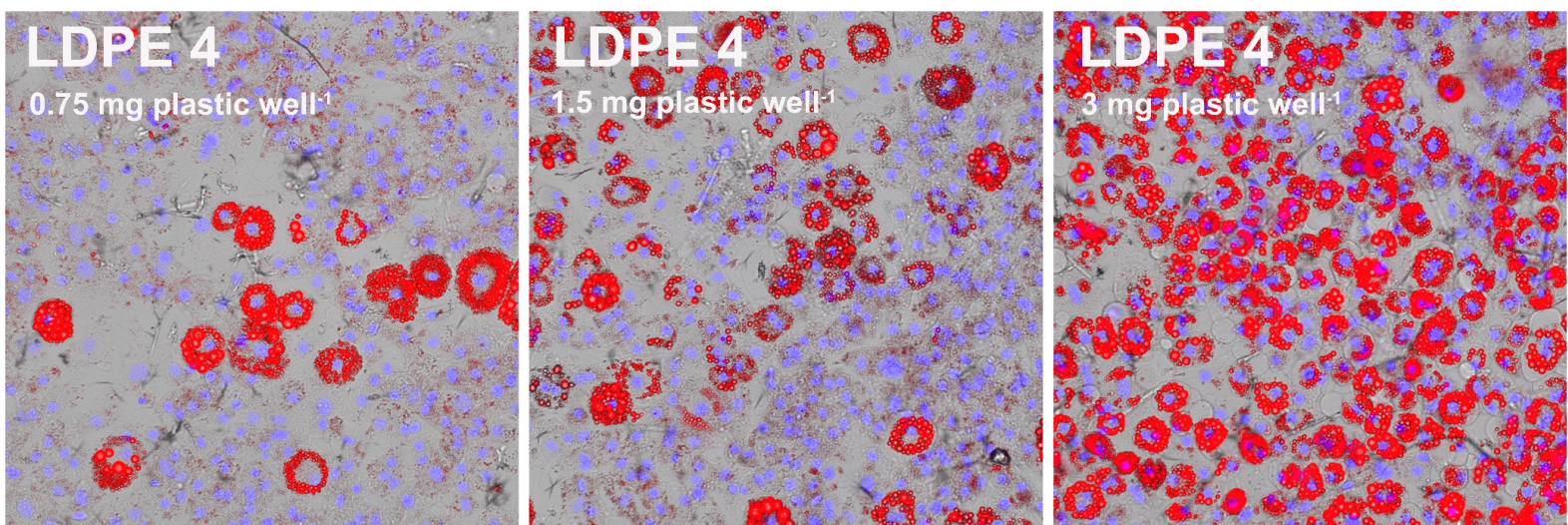

Figure S10. Dose-dependent induction of adipogenesis in 3T3-L1 cells exposed to the active plastic extract LDPE 4. Merged brightfield and fluorescence images. Nuclei are stained with NucBlue (blue) and triglycerides with NileRed (red). Raw pictures were processed in the same manner for visualization.
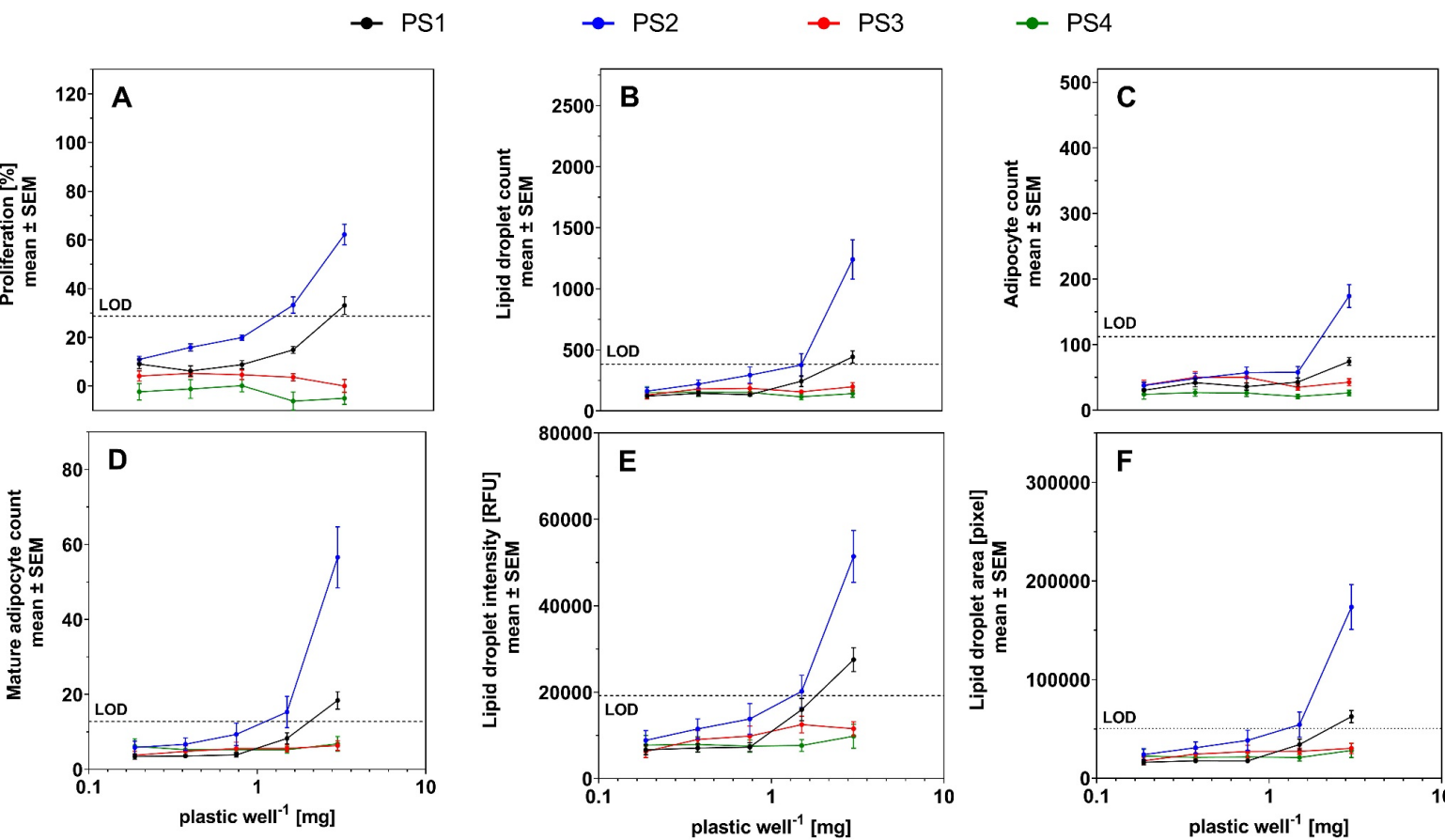

Figure S11. Dose-response relationships for the PS plastic extracts (PS 1-4) in the

287 adipogenesis assay. (A) proliferation normalized on the mean of the vehicle control, (B) lipid 288 droplet count per field, (C) adipocyte count per field, (D) mature adipocyte count per field, (E) 289 total intensity of the NileRed staining within the lipid droplet mask per field and (F) total area 290 occupied by lipid droplets per field. Twelve or more replicates per concentration $(n \geq 12)$. LOD $291=$ limit of detection, RFU = relative fluorescence unit. 

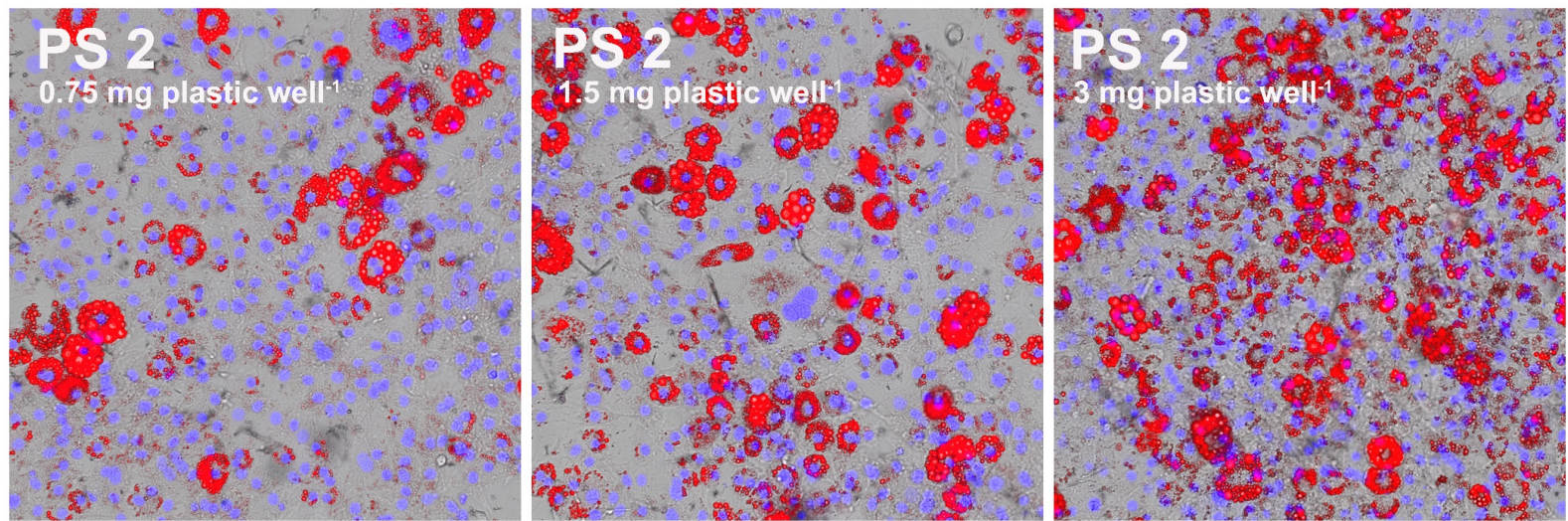

Figure S12. Dose-dependent induction of adipogenesis in 3T3-L1 cells exposed to the active plastic extract PS 2. Merged brightfield and fluorescence images. Nuclei are stained with NucBlue (blue) and triglycerides with NileRed (red). Raw pictures were processed in the same manner for visualization.
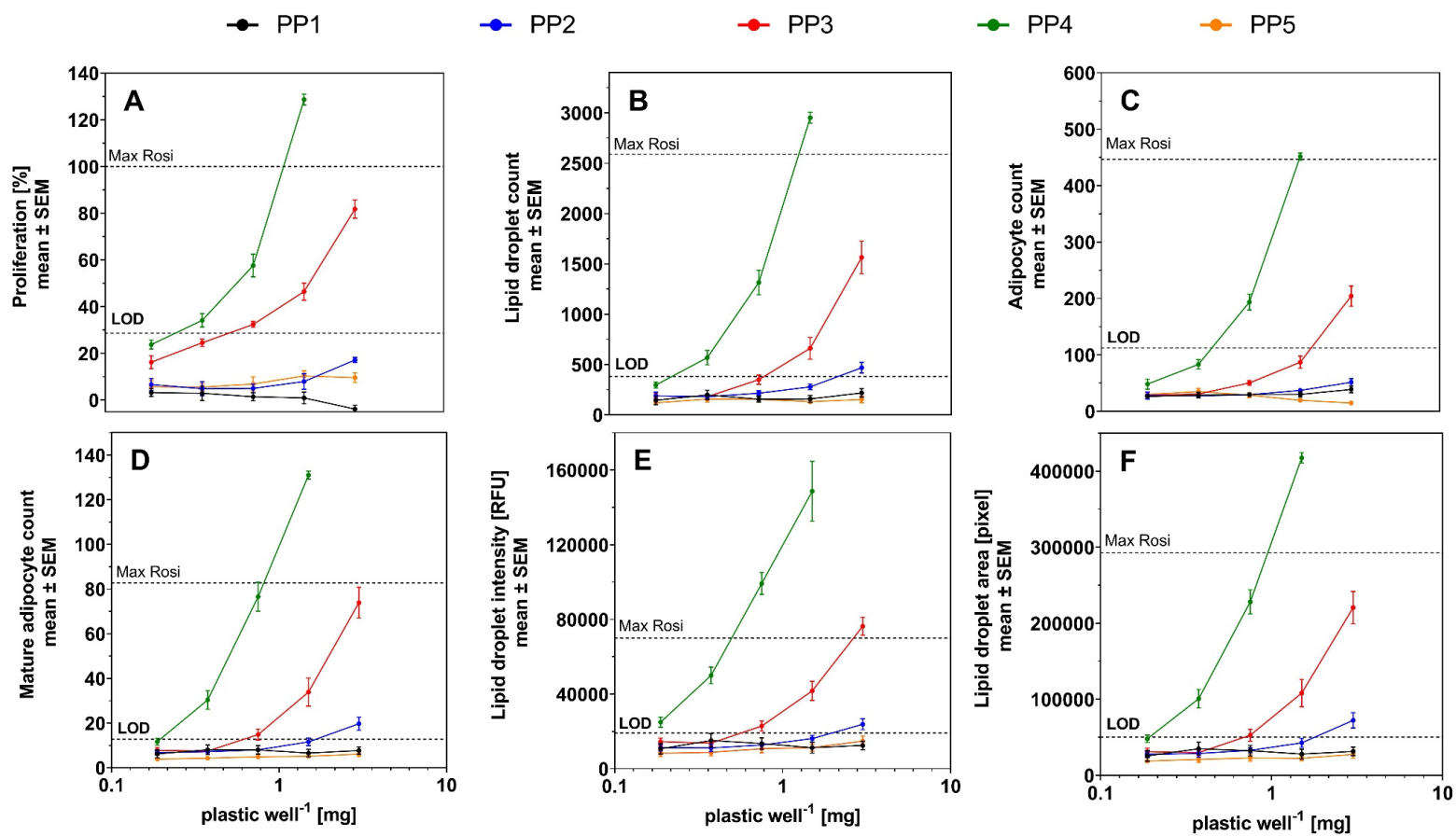

Figure S13. Dose-response relationship for the PP plastic extracts (PP 1-5) in the adipogenesis assay. (A) proliferation normalized on the mean of the vehicle control, (B) lipid droplet count per field, (C) adipocyte count per field, (D) mature adipocyte count per field, (E) total intensity of the NileRed staining within the lipid droplet mask per field and (F) total area occupied by lipid droplets per field. Twelve or more replicates per concentration ( $\mathrm{n} \geq 12)$. LOD $=$ limit of detection, Max Rosi $=$ rosiglitazone maximal response, $\mathrm{RFU}=$ relative fluorescence unit. 



307 Figure S14. Dose-dependent induction of adipogenesis in 3T3-L1 cells exposed to the

308 active plastic extracts PP 3 and PP 4. Merged brightfield and fluorescence images. Nuclei

309 are stained with NucBlue (blue) and triglycerides with NileRed (red). Raw pictures were

310 processed in the same manner for visualization. 

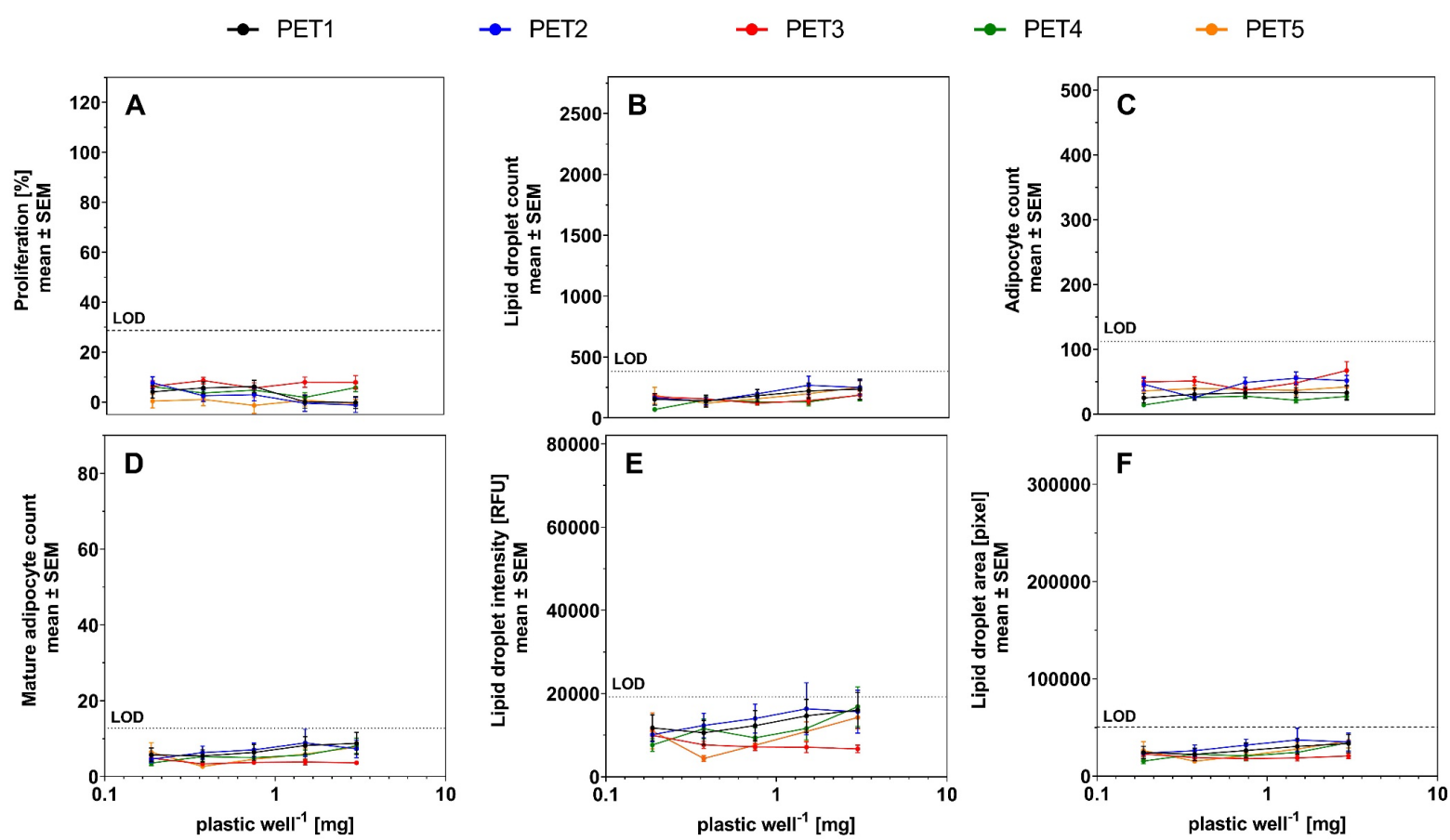

312 Figure S15. Dose-response relationship for the PET plastic extracts (PET 1-5) in the

313 adipogenesis assay. (A) proliferation normalized on the mean of the vehicle control, (B) lipid 314 droplet count per field, (C) adipocyte count per field, (D) mature adipocyte count per field, (E) 315 total intensity of the NileRed staining within the lipid droplet mask per field and (F) total area 316 occupied by lipid droplets per field. Twelve or more replicates per concentration $(n \geq 12)$. LOD $317=$ limit of detection, $\mathrm{RFU}=$ relative fluorescence unit. 

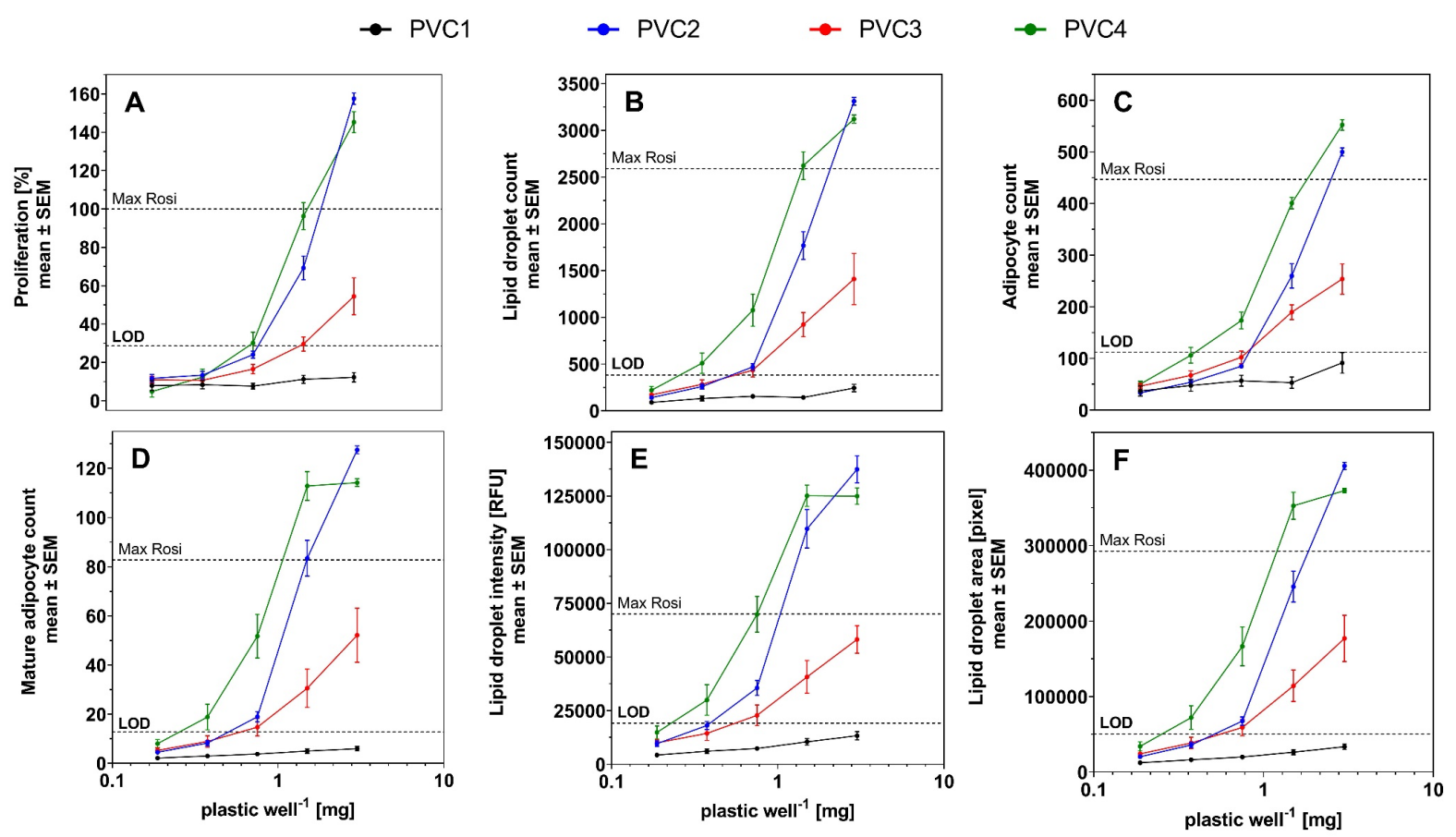

320 Figure S16. Dose-response relationship for the PVC plastic extracts (PVC 1-4) in the

321 adipogenesis assay. (A) proliferation normalized on the mean of the vehicle control, (B) lipid 322 droplet count per field, (C) adipocyte count per field, (D) mature adipocyte count per field, (E) 323 total intensity of the NileRed staining within the lipid droplet mask per field and (F) total area 324 occupied by lipid droplets per field. Twelve or more replicates per concentration $(n \geq 12)$. LOD $325=$ limit of detection, Max Rosi = rosiglitazone maximal response, RFU = relative fluorescence 326 unit. 

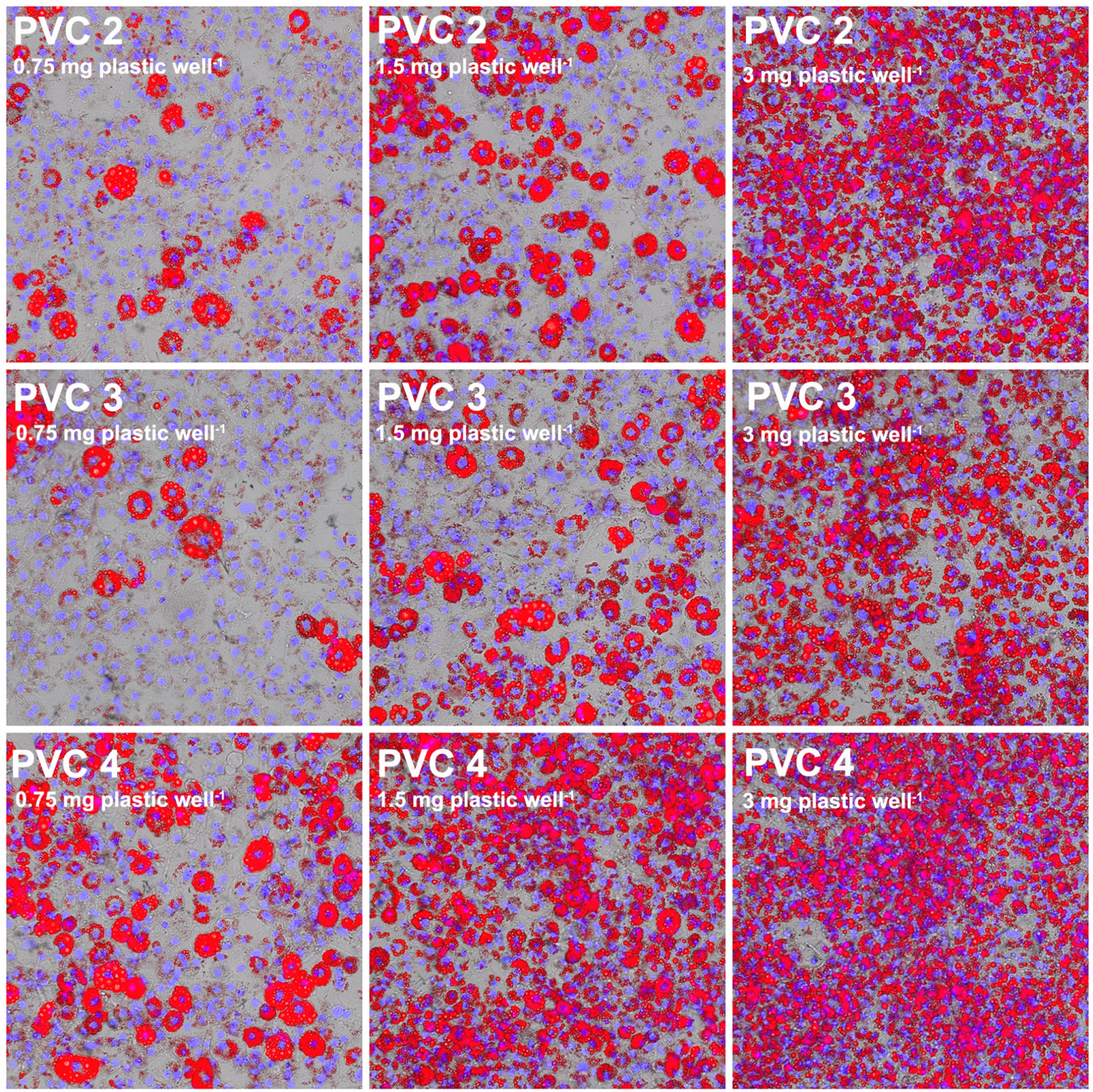

328 Figure S17. Dose-dependent induction of adipogenesis in 3T3-L1 cells exposed to the

329 active plastic extracts PVC 2- 4. Merged brightfield and fluorescence images. Nuclei are

330 stained with NucBlue (blue) and triglycerides with NileRed (red). Raw pictures were processed 331 in the same manner for visualization. 

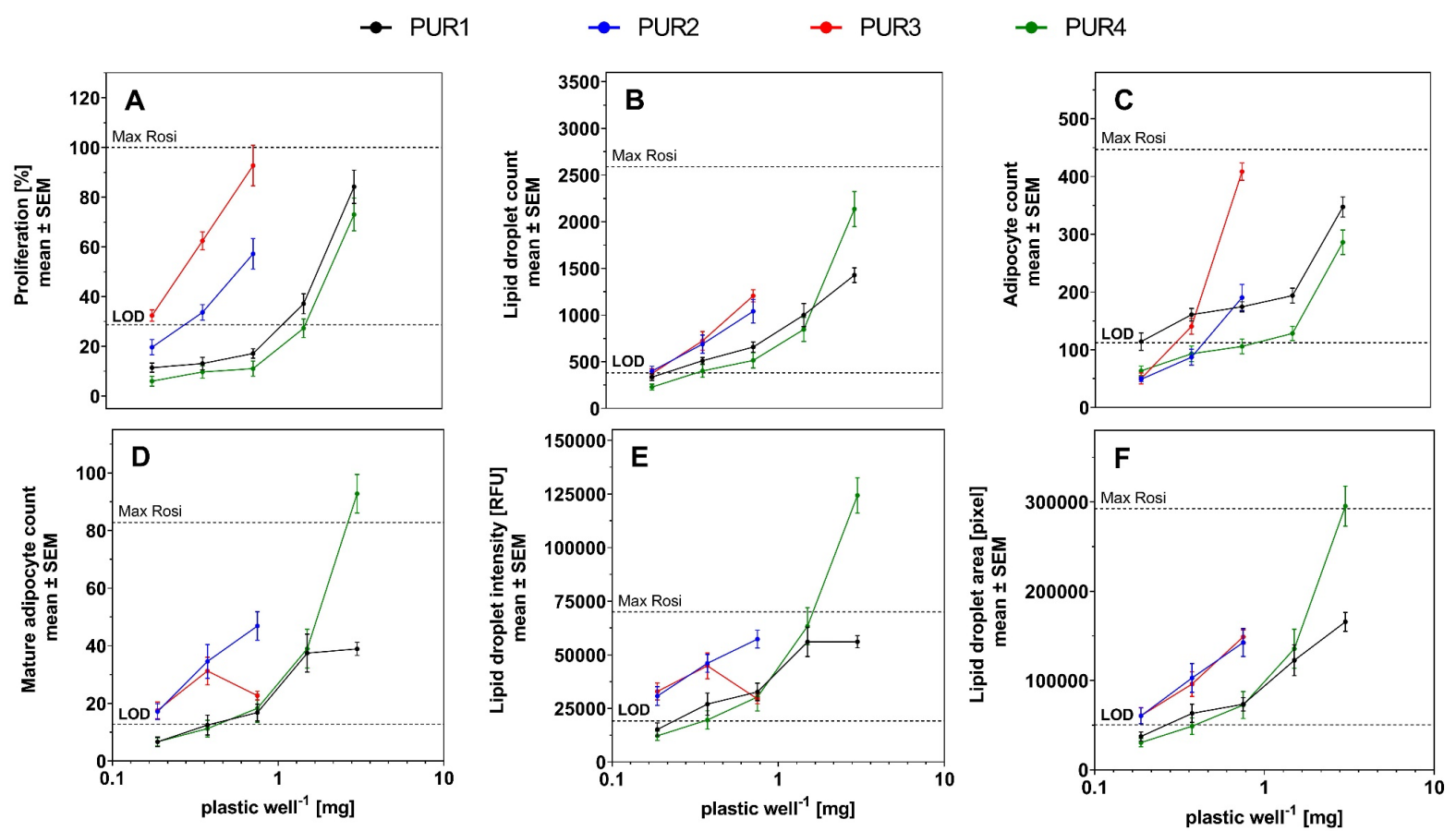

Figure S18. Dose-response relationship for the PUR plastic extracts (PUR 1-4) in the adipogenesis assay. (A) proliferation normalized on the mean of the vehicle control, (B) lipid droplet count per field, (C) adipocyte count per field, (D) mature adipocyte count per field, (E) total intensity of the NileRed staining within the lipid droplet mask per field and (F) total area

338 occupied by lipid droplets per field. Twelve or more replicates per concentration $(n \geq 12)$. LOD

$339=$ limit of detection, Max Rosi = rosiglitazone maximal response, RFU = relative fluorescence 340 unit. 

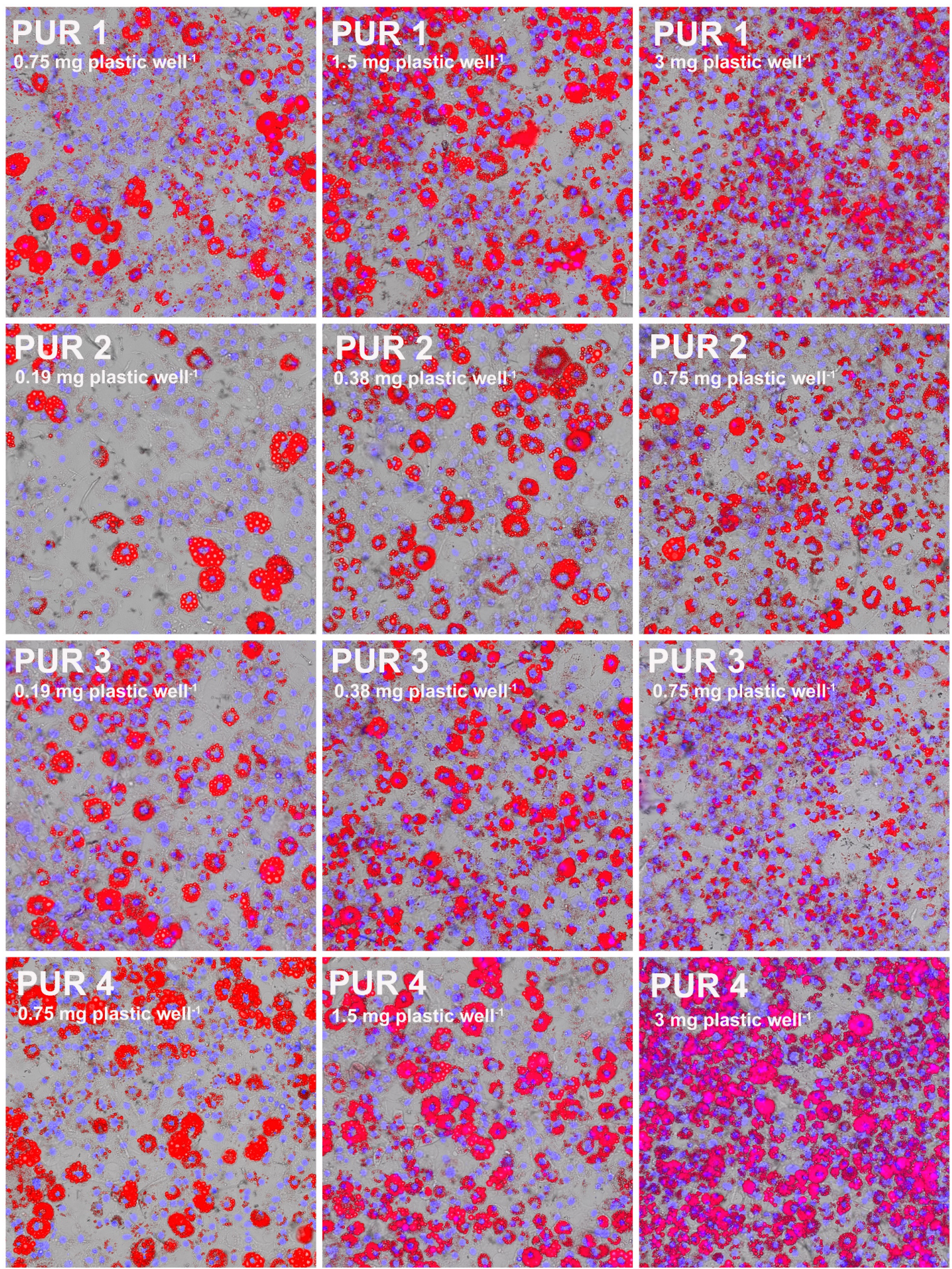

342 Figure S19. Dose-dependent induction of adipogenesis in 3T3-L1 cells exposed to the

343 active plastic extracts PUR 1-4. Merged brightfield and fluorescence images. Nuclei are 344 stained with NucBlue (blue) and triglycerides with NileRed (red). Raw pictures were processed 345 in the same manner for visualization. 

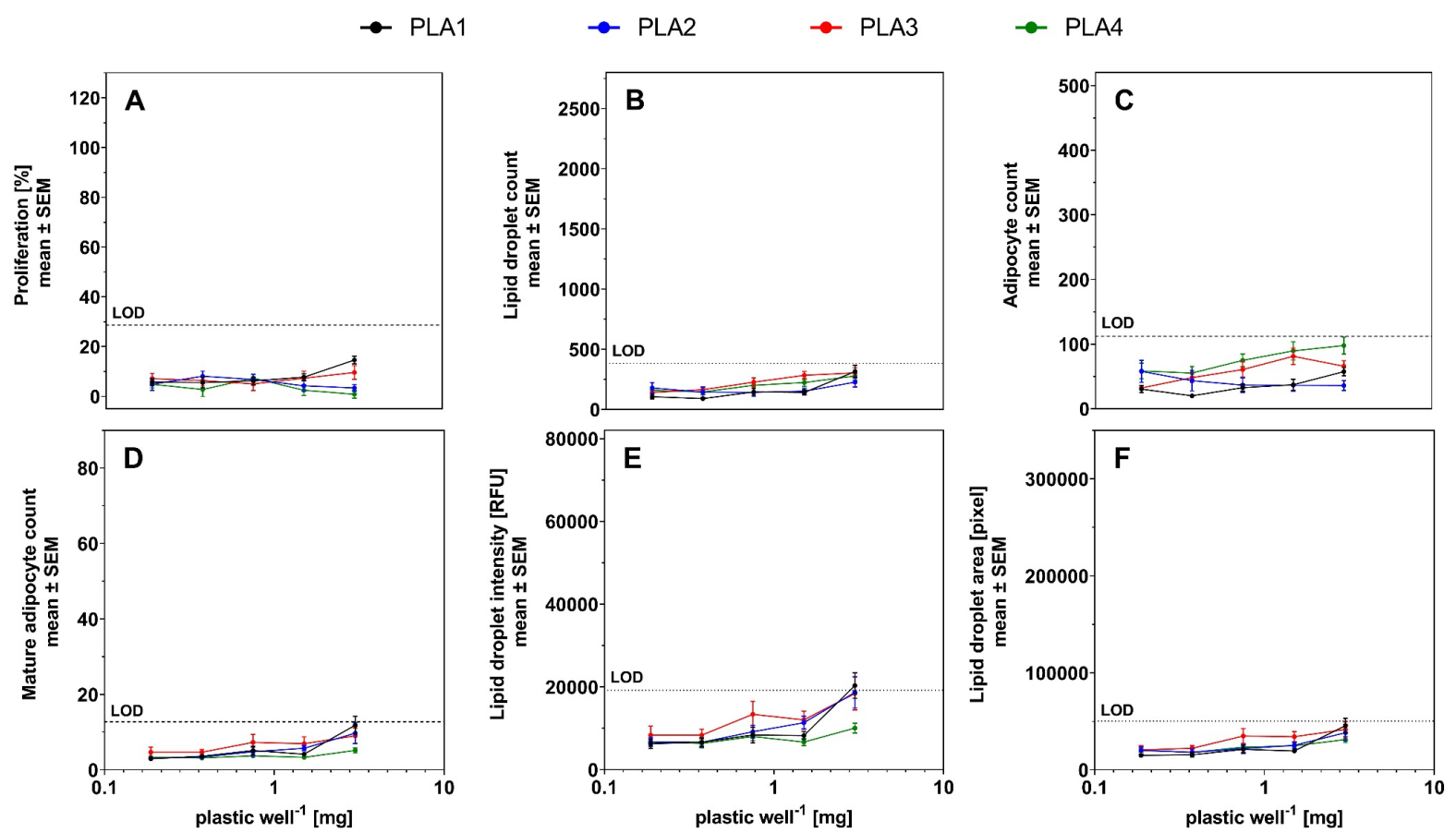

348 Figure S20. Dose-response relationship for the PLA plastic extracts (PLA 1-4) in the

349 adipogenesis assay. (A) proliferation normalized on the mean of the vehicle control, (B) lipid 350 droplet count per field, (C) adipocyte count per field, (D) mature adipocyte count per field, (E) 351 total intensity of the NileRed staining within the lipid droplet mask per field and (F) total area 352 occupied by lipid droplets per field. Twelve or more replicates per concentration $(n \geq 12)$. LOD $353=$ limit of detection, Max Rosi = rosiglitazone maximal response, RFU = relative fluorescence 354 unit. 

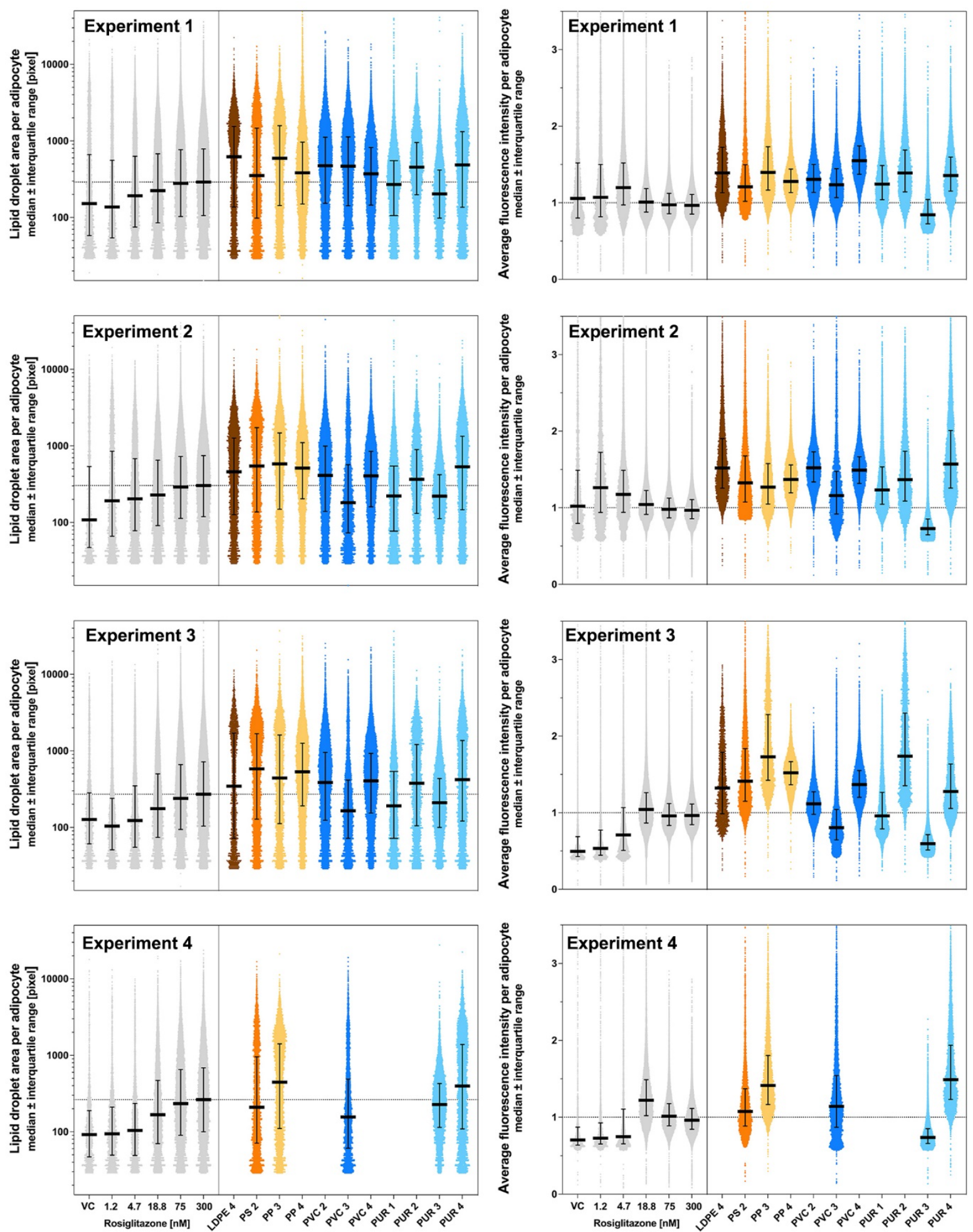

Figure S21. Size distribution of adipocyte population (left) and accumulation of triglyceride per adipocyte in cells exposed to rosiglitazone or the highest noncytotoxic concentration of the eleven active plastic extracts. Single-cell data from 4 independent experiments. Intensity data is normalized to the mean of the highest rosiglitazone concentration $(300 \mathrm{nM}) . \mathrm{VC}=$ vehicle control. 


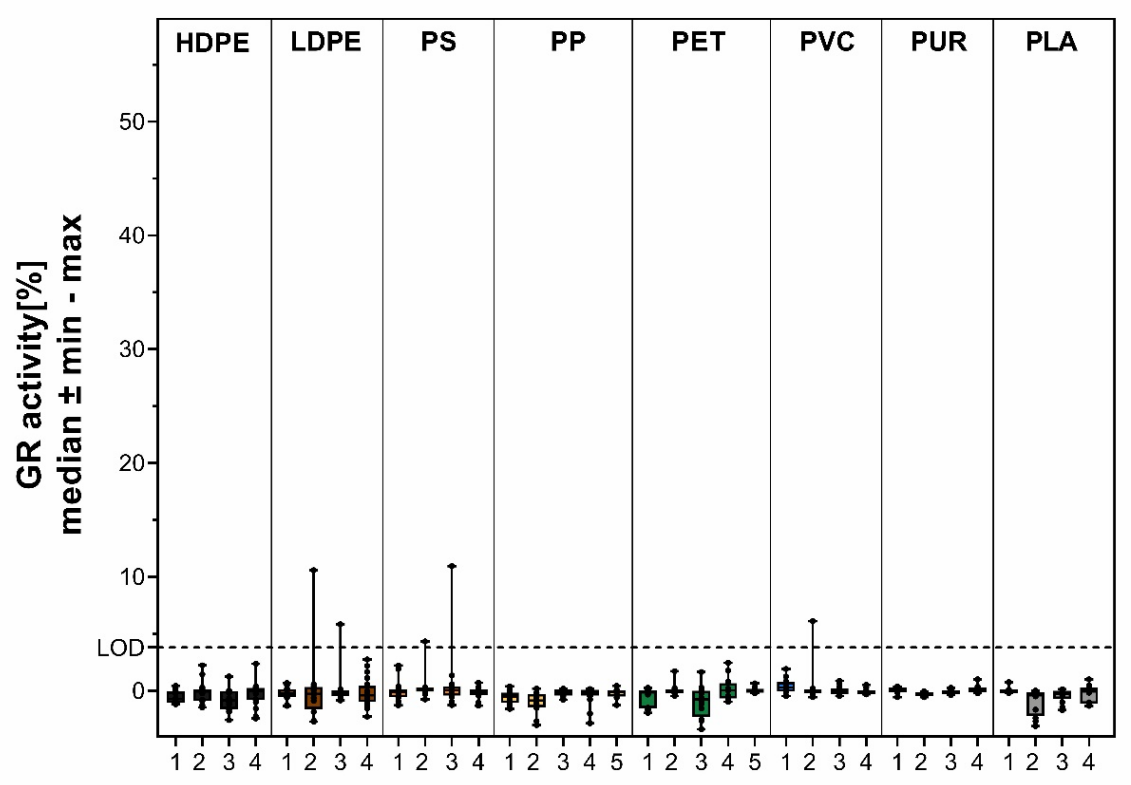

362 Figure S22. GR activity of plastic extracts at the highest noncytotoxic concentration.

363 Highest noncytotoxic concentration was $1.5 \mathrm{mg}$ plastic well-1 except for PP 4 (0.19 mg plastic 364 well-1), PS 2 and PP 3 (0.38 mg plastic well-1), PLA 1, PVC 2 and PVC 4 (0.75 mg plastic 365 well-1). GR = glucocorticoid receptor, $\mathrm{LOD}=$ limit of detection. $\mathrm{n} \geq 12$.

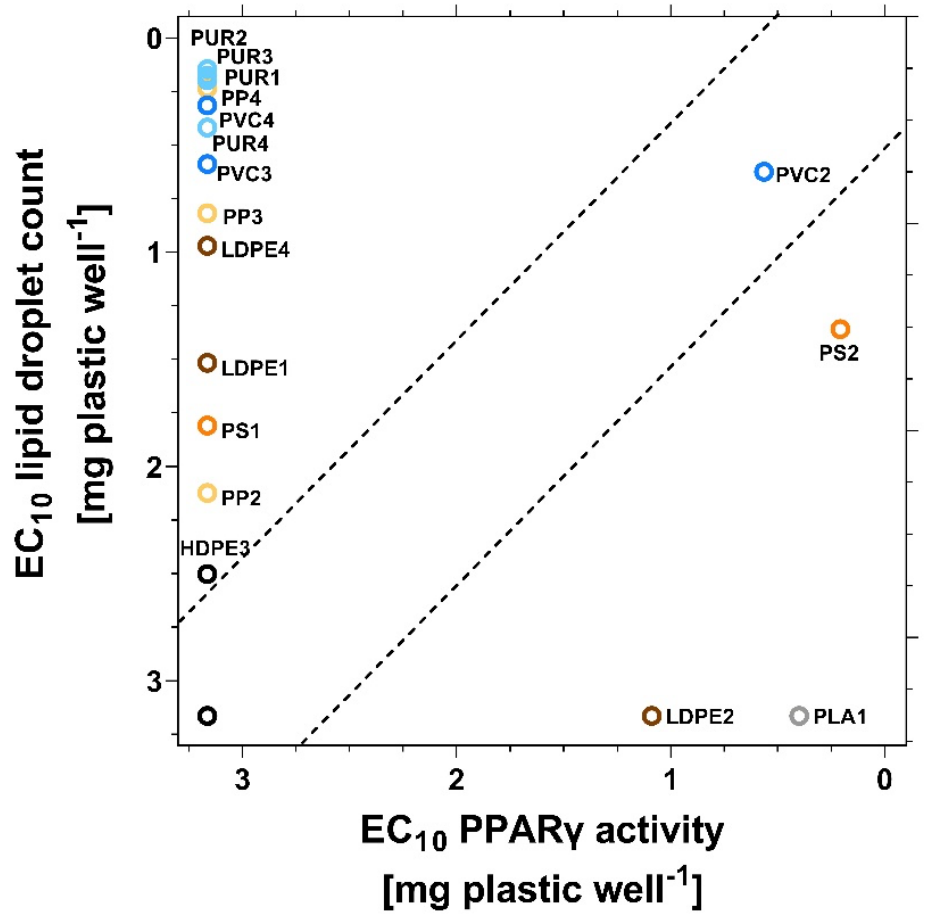

367 Figure S23. Correlation of the $\mathrm{EC}_{10}$ of the PPAR $\gamma$ activity and lipid droplet count. 

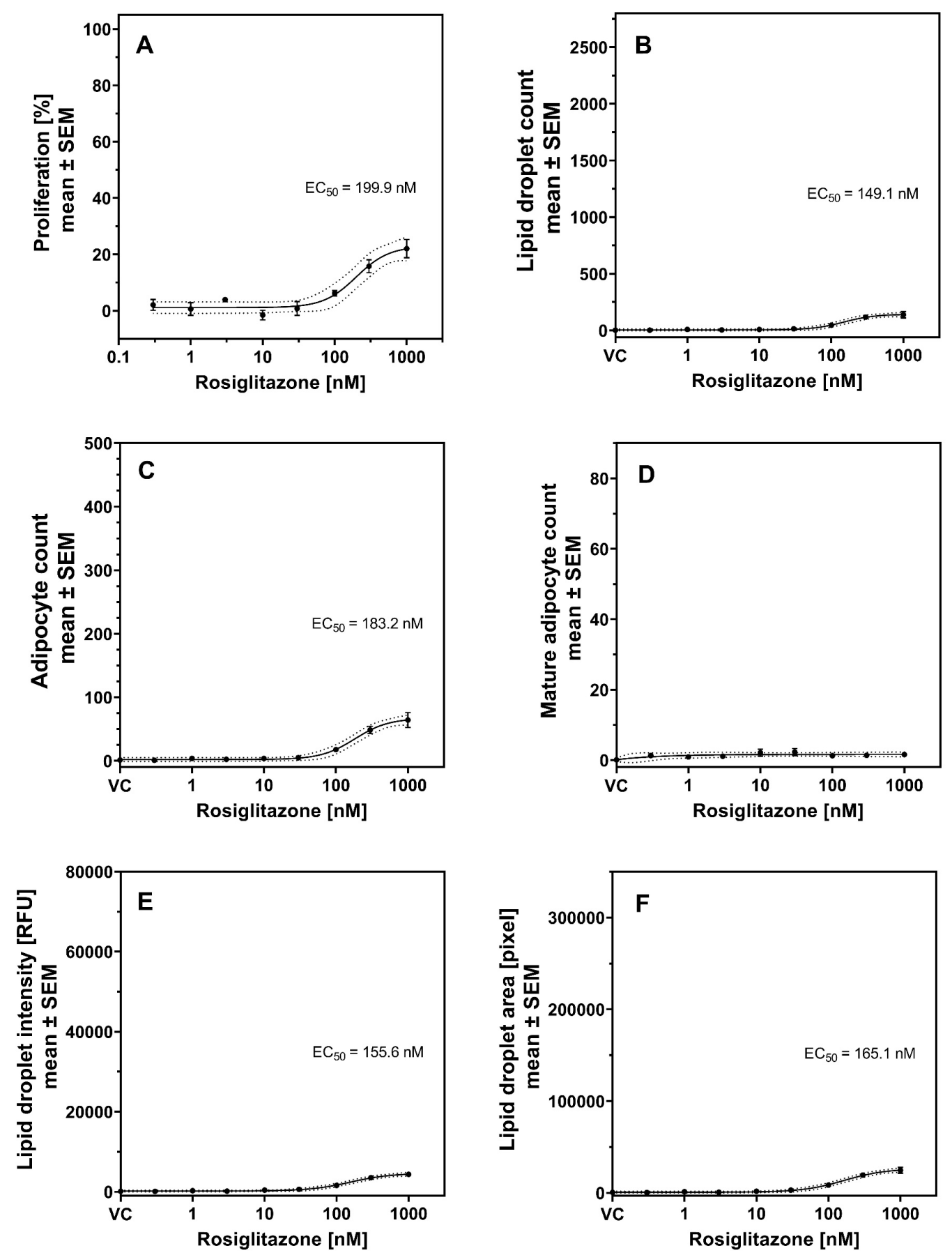

369 Figure S24. Dose-response relationship for the reference compound rosiglitazone in the

370 adipogenesis assay without dexamethasone in the differentiation medium. (A)

371 proliferation normalized on the mean of the vehicle control, (B) lipid droplet count per field,

372 (C) adipocyte count per field, (D) mature adipocyte count per field, (E) total intensity of the

373 NileRed staining within the lipid droplet mask per field and (F) total area occupied by lipid 374 droplets per field. Eight or more replicates per concentration $(\mathrm{n} \geq 8) . \mathrm{VC}=$ vehicle control, $375 \quad \mathrm{RFU}=$ relative fluorescence unit. 
Table S1. List of all used chemicals and consumables.

\begin{tabular}{|c|c|c|}
\hline chemical/consumable & supplier & further information \\
\hline AdipoRed assay kit (NileRed) & Lonza & N3013 \\
\hline ATP (adenosine-5'-triphosphate disodium salt) & PanReac AppliChem & $\geq 98 \%$ (HPLC), CAS: 987655 \\
\hline Bovine calf serum iron-supplemented & Sigma & $12138 \mathrm{C}$ \\
\hline Charcoal-stripped fetal bovine serum & Gibco & 12676029 \\
\hline $\begin{array}{l}\text { CDTA (1,2-cyclohexanedinitrilotetraacetic } \\
\text { acid) }\end{array}$ & Sigma & $\geq 99 \%$ (KT), CAS: 125572954 \\
\hline Dexamethasone & Sigma & $\geq 98 \%$ (HPLC), CAS: 50022 \\
\hline DMEM/F-12 with phenol red & Gibco & 31331093 \\
\hline DMEM/F-12 without phenol red & Gibco & 21041025 \\
\hline DMEM high glucose & Gibco & 31966047 \\
\hline D-Luciferin, monopotassium salt & Thermo Scientific & $\geq 99.7 \%$ (HPLC), 88294 \\
\hline DL-dithiothreitol & Sigma & $\geq 98 \%$ (HPLC), CAS: 3483123 \\
\hline DMSO (dimethyl sulfoxide) & Sigma & $\geq 99.5 \%$ (GC), CAS: 67685 \\
\hline EDTA (ethylenediaminetetraacetic acid) & Sigma & $\geq 99 \%$ (titration), CAS: 60004 \\
\hline Fetal bovine serum & Gibco & $10270-106$ \\
\hline G 418 disulfate & Sigma & $\geq 450 \mathrm{U} / \mathrm{mg}, \mathrm{CAS}: 108321422$ \\
\hline Glycerol & Sigma & $\geq 99 \%$ (GC), CAS: 56815 \\
\hline HEPES & VWR & L1613 \\
\hline IBMX (3-isobutyl-1-methylxanthine) & Sigma & $\geq 99 \%$ (HPLC), CAS: 28822587 \\
\hline Insulin, human recomb., zinc solution & Gibco & $12585014,27 \mathrm{U} / \mathrm{mg}$ \\
\hline Magnesium carbonate hydroxide pentahydrate & Sigma & M5671, CAS: 56378724 \\
\hline Magnesium sulfate heptahydrate & Sigma & $\geq 98 \%$, CAS: 10034998 \\
\hline Methanol & Sigma & $\geq 99.8 \%$ (HPLC), 322415 \\
\hline NucBlue live cell ready probes reagent & Life technologies & R37605 \\
\hline Penicillin/streptomycin & VWR & L0022-100 \\
\hline Rosiglitazone & Sigma & $\geq 98 \%$ (HPLC), CAS: 122320734 \\
\hline Sterile T75 cell culture flasks & Thermo Scientific & 156499 \\
\hline Tris base & Sigma & $\geq 99 \%$ (titration), CAS: 77861 \\
\hline Triton $^{\mathrm{TM}} \mathrm{X}-100$ & Sigma & laboratory grade, CAS: 9002-93-1 \\
\hline $\begin{array}{l}\text { 96-well cell culture plates (black with } \\
\text { transparent bottom) }\end{array}$ & Greiner & Cellstar 655090 \\
\hline $\begin{array}{l}\text { 384-well cell culture plates (white with } \\
\text { transparent bottom) }\end{array}$ & Greiner & Cellstar 738-0062 \\
\hline
\end{tabular}


378 Table S2. List of adipogenic chemicals based on the published literature.

\begin{tabular}{|c|c|c|c|}
\hline Name & CAS & PubChem CID & References \\
\hline $1-850$ & $251310-57-3$ & 2765122 & 4 \\
\hline 2,4,6-tribromophenol & $118-79-6$ & 1483 & 15 \\
\hline $\begin{array}{l}\text { 2-ethylhexyl diphenyl } \\
\text { phosphate }\end{array}$ & $1241-94-7$ & 14716 & 16 \\
\hline 3,5,6-trichloro-2-pyridinol & $6515-38-4$ & 23017 & 17 \\
\hline 3-tert-butyl-4-hydroxanisole & $121-00-6$ & 8456 & 13 \\
\hline 4-hexyl phenol & $2446-69-7$ & 17132 & 18 \\
\hline 4-n-octylphenol & $1806-26-4$ & 15730 & 19 \\
\hline 4-nonylphenol & $104-40-5$ & 1752 & 19,20 \\
\hline 8:2 FTAcr & $27905-45-9$ & 119747 & 15 \\
\hline Acetamiprid & $135410-20-7$ & 213021 & 21 \\
\hline Acrylamide & $79-06-1$ & 6579 & 13 \\
\hline Allethrin & $584-79-2$ & 11442 & 22 \\
\hline Alpha naphthoflavone & $604-59-1$ & 11790 & 13 \\
\hline Azoxystrobin & $131860-33-8$ & 3034285 & 15 \\
\hline BDE-47 & $5436-43-1$ & 95170 & 15,23 \\
\hline Benzyl butyl phthalate & $85-68-7$ & 2347 & $24-26$ \\
\hline Biphenthrin & $82657-04-03$ & 6442842 & 27 \\
\hline Bisphenol A & $80-05-07$ & 6623 & $21,23-26,28-33$ \\
\hline Bisphenol A diglycidyl ether & $1675-54-3$ & 2286 & 31 \\
\hline Bisphenol F & $620-92-8$ & 12111 & 28,29 \\
\hline Bisphenol S & $80-09-1$ & 6626 & $28-30,32$ \\
\hline Butylparaben & $94-26-8$ & 7184 & 26 \\
\hline Carboxymethylcellulose & $9000-11-7$ & 24748 & 13 \\
\hline Cetyl alcohol ethoxylate & $5274-61-3$ & 4303686 & 19 \\
\hline Chlorpyrifos & $2921-88-2$ & 2730 & 15,17 \\
\hline Cypermethrin & $52315-07-8$ & 2912 & 15 \\
\hline Daidzein & $486-66-8$ & 5281708 & 34 \\
\hline DDE & $72-55-9$ & 3035 & 13 \\
\hline DDT & $50-29-3$ & 3036 & 13 \\
\hline Dechlorane plus & $13560-89-9$ & 26111 & 35 \\
\hline DEHP & $117-81-7$ & 8343 & 15,36 \\
\hline Dexamethasone & $50-02-2$ & 5743 & $4,37,38$ \\
\hline Diazinon & $333-41-5$ & 3017 & 39 \\
\hline Diethylene glycol dibenzoate & $120-55-8$ & 8437 & 6 \\
\hline Dibutyl phthalate & $84-74-2$ & 3026 & 15,26 \\
\hline Dibutyltin & $1002-53-5$ & 6484 & 13 \\
\hline Dicamba & 1918-00-9 & 3030 & 37 \\
\hline Diclofop-methyl & $51338-27-3$ & 39985 & 13 \\
\hline
\end{tabular}




\begin{tabular}{|c|c|c|c|}
\hline Name & CAS & PubChem CID & References \\
\hline Di-iso-butyl phthalate & $84-69-5$ & 6782 & 26 \\
\hline Di-iso-decyl phthalate & $89-16-7$ & 33599 & 6 \\
\hline Di-iso-nonyl phthalate & $28553-12-0$ & 590836 & 6 \\
\hline $\begin{array}{l}\text { Dioctyl sodium } \\
\text { sulfosuccinate }\end{array}$ & $577-11-7$ & 23673837 & 40,41 \\
\hline Diphenyl phosphate & $838-85-7$ & 13282 & 42 \\
\hline Ethylparaben & $120-47-8$ & 8434 & 26 \\
\hline Fenthion & $55-38-9$ & 3346 & 22 \\
\hline Fludioxonil & $131341-86-1$ & 86398 & 21 \\
\hline Fluoxastrobin & $361377-29-9$ & 11048796 & 15 \\
\hline Flusilazole & $85509-19-9$ & 73675 & 21 \\
\hline Flutamide & $13311-84-7$ & 3397 & 4 \\
\hline Forchlorfenuron & $68157-60-8$ & 93379 & 21 \\
\hline Genistein & $446-72-0$ & 5280961 & 34 \\
\hline Glyphosate & $1071-83-6$ & 3496 & 13 \\
\hline GW3965 & $405911-17-3$ & 16078973 & 4 \\
\hline Halosulfuron-methyl & $100784-20-1$ & 91763 & 13 \\
\hline HBCD & $3194-55-6$ & 18529 & 43 \\
\hline Hexafluorobisphenol A & $1478-61-1$ & 73864 & 4 \\
\hline Imidacloprid & $138261-41-3$ & 86287518 & 38 \\
\hline $\begin{array}{l}\text { Isopropylated triphenyl } \\
\text { phosphate }\end{array}$ & $78-30-8$ & 6527 & 44 \\
\hline Isoxaflutole & $141112-29-0$ & 84098 & 37 \\
\hline Lactofen & $77501-63-4$ & 62276 & 13 \\
\hline Lauryl alcohol ethoxylate (4) & $4536-30-5$ & 24750 & 19 \\
\hline LG100268 & $153559-76-3$ & 3922 & 4 \\
\hline MEHP & $4376-20-9$ & 20393 & 36 \\
\hline Methylparaben & $99-76-3$ & 7456 & 26,45 \\
\hline Mono(2-ethylhexyl) phthalate & $4376-20-9$ & 20393 & 46 \\
\hline Monosodium glutamate & $142-47-2$ & 23672308 & 13 \\
\hline Musk xylene & $81-15-2$ & 62329 & 26 \\
\hline Nonylphenol ethoxylate (1-2) & $9016-45-9$ & 24773 & 19 \\
\hline Nonylphenol ethoxylate (20) & $\mathrm{N} / \mathrm{A}$ & $\mathrm{N} / \mathrm{A}$ & 19 \\
\hline Nonylphenol ethoxylate (4) & N/A & $\mathrm{N} / \mathrm{A}$ & 19 \\
\hline Nonylphenol ethoxylate (6) & N/A & $\mathrm{N} / \mathrm{A}$ & 19 \\
\hline $\begin{array}{l}\text { Nonylphenol ethoxylate (9- } \\
10)\end{array}$ & N/A & N/A & 19 \\
\hline Octylphenol ethoxylate (3) & $2315-67-5$ & 5590 & 19 \\
\hline P-80 & $9005-65-6$ & 5284448 & 13 \\
\hline PBDE 99 & $60348-60-9$ & 36159 & 47 \\
\hline PCB 153 & $35065-27-1$ & 37034 & 23 \\
\hline
\end{tabular}




\begin{tabular}{|c|c|c|c|}
\hline Name & CAS & PubChem CID & References \\
\hline Permethrin & $52645-53-1$ & 40326 & 15 \\
\hline PFHxS & $3871-99-6$ & 23678874 & 48 \\
\hline PFNA & $375-95-1$ & 67821 & 48 \\
\hline PFOA & $335-67-1$ & 9554 & $23,48,49$ \\
\hline PFOS & $1763-23-1$ & 74483 & 48 \\
\hline Pioglitazone & $112529-15-4$ & 60560 & 22 \\
\hline Pirinixic acid & $50892-23-4$ & 5694 & 48 \\
\hline Prallethrin & $23031-36-9$ & 9839306 & 22 \\
\hline Propylparaben & $94-13-3$ & 7175 & 26,45 \\
\hline Pymetrozine & $123312-89-0$ & 9576037 & 21 \\
\hline Pyraclostrobin & $175013-18-0$ & 6422843 & 15 \\
\hline Pyrimethanil & $53112-28-0$ & 91650 & 22 \\
\hline Quinoxyfen & $124495-18-7$ & 3391107 & 21,22 \\
\hline Quizalofop-p-ethyl & $100646-51-3$ & 1617113 & 37 \\
\hline Retinoic acid & $302-79-4$ & 444795 & 22 \\
\hline Rosiglitazone & $122320-73-4$ & 77999 & Positive control \\
\hline Span 80 & $1338-43-8$ & 9920342 & 41 \\
\hline Spirodiclofen & $148477-71-8$ & 177863 & 21 \\
\hline TBEP & $78-51-3$ & 6540 & 15 \\
\hline ТВРH & $26040-51-7$ & 117291 & 15 \\
\hline TDBPIC & $52434-90-9$ & 103634 & 15 \\
\hline Tebuconazole & $107534-96-3$ & 86102 & 22 \\
\hline Tebupirimfos & $96182-53-5$ & 93516 & 21 \\
\hline $\begin{array}{l}\text { Tert-butylphenyl diphenyl } \\
\text { phosphate }\end{array}$ & $56803-37-3$ & 158333 & 15 \\
\hline Tetrabromobisphenol A & $79-94-7$ & 6618 & $2,4,22$ \\
\hline Tetrachlorobisphenol A & $79-95-8$ & 6619 & 4 \\
\hline Tolyfluanid & $731-27-1$ & 12898 & 50 \\
\hline Tomadol 1-9 & N/A & N/A & 19 \\
\hline Tonalide & $1506-02-1$ & 89440 & 26 \\
\hline Tributyltin & $687-73-3$ & 3032732 & $4,23,26,51-56$ \\
\hline Triclocarban & $101-20-2$ & 7547 & 57 \\
\hline $\begin{array}{l}\text { Tridecyl alcohol ethoxylate } \\
\text { (9) }\end{array}$ & $78330-21-9$ & $\mathrm{~N} / \mathrm{A}$ & 19 \\
\hline Trifloxystrobin & $141517-21-7$ & 11665966 & 15 \\
\hline Triflumizole & $68694-11-1$ & 91699 & 21,58 \\
\hline Tri-m-cresyl phosphate & $563-04-2$ & 11232 & 6 \\
\hline Tri-n-butyl phosphate & $126-73-8$ & 31357 & 15 \\
\hline Triphenyl phosphate & $115-86-6$ & 8289 & $15,42,44,59$ \\
\hline Triphenyltin & $76-87-9$ & 6327657 & 21,22 \\
\hline
\end{tabular}




\begin{tabular}{lrrr}
\hline Name & \multicolumn{1}{c}{ CAS } & PubChem CID & References \\
\hline Tris(4-tert-butylphenyl) & $78-33-1$ & 6530 & 15 \\
phosphate & & & \\
Troglitazone & $97322-87-7$ & 5591 & $23,25,44$ \\
TTBP & $732-26-3$ & 12902 & 22 \\
Zoxamide & $156052-68-5$ & 122087 & 21 \\
\hline
\end{tabular}

379 
380 Table S3. The chemicals tentatively identified using GC-QTOF-MS/MS (data from Zimmermann et al. $)^{14}$

\begin{tabular}{|c|c|c|c|c|c|c|}
\hline Sample & CAS & $\begin{array}{l}\text { PubChem } \\
\text { CID }\end{array}$ & Name according to NIST & Mass & Formula & Score \\
\hline \multirow[t]{5}{*}{ HDPE 1} & $1000374-06-1^{a}$ & 3023652 & 1,7-di-iso-propylnaphthalene & 212.16 & $\mathrm{C} 16 \mathrm{H} 20$ & 71.58 \\
\hline & $10233-13-3$ & 25068 & Dodecanoic acid, 1-methylethyl ester & 242.23 & $\mathrm{C} 15 \mathrm{H} 30 \mathrm{O} 2$ & 72.54 \\
\hline & $138345-00-3$ & 605776 & 7,9-Di-tertbutyl-1-oxaspiro[4,5]deca-6,9-dien-8-one & 262.19 & $\mathrm{C} 17 \mathrm{H} 26 \mathrm{O} 2$ & 70.98 \\
\hline & $24169-43-5$ & 595960 & 1,3-Dioxolane, 2-(bromomethyl)-2-(2-methylphenyl)- & 256.01 & $\mathrm{C} 11 \mathrm{H} 13 \mathrm{BrO} 2$ & 72.61 \\
\hline & $87-97-8$ & 6911 & Phenol, 2,6-bis(1,1-dimethylethyl)-4-(methoxymethyl)- & 250.19 & $\mathrm{C} 16 \mathrm{H} 26 \mathrm{O} 2$ & 70.81 \\
\hline \multirow[t]{2}{*}{ HDPE 2} & $4337-65-9$ & 20342 & Hexanedioic acid, mono(2-ethylhexyl)ester & 258.18 & $\mathrm{C} 14 \mathrm{H} 26 \mathrm{O} 4$ & 82.22 \\
\hline & $6386-38-5$ & 62603 & Benzenepropanoic acid, 3,5-bis(1,1-dimethylethyl)-4-hydroxy-, methyl ester & 292.20 & $\mathrm{C} 18 \mathrm{H} 28 \mathrm{O} 3$ & 71.02 \\
\hline HDPE 3 & $96-76-4$ & 7311 & 2,4-Di-tert-butylphenol & 206.17 & $\mathrm{C} 14 \mathrm{H} 22 \mathrm{O}$ & 80.64 \\
\hline \multirow[t]{15}{*}{ HDPE 4} & $1000192-65-0^{a}$ & 543423 & 1,2-15,16-Diepoxyhexadecane & 254.23 & $\mathrm{C} 16 \mathrm{H} 30 \mathrm{O} 2$ & 72.96 \\
\hline & $1000315-44-3^{a}$ & 6423518 & Phthalic acid, isobutyl tridec-2-yn-1-yl ester & 400.26 & $\mathrm{C} 25 \mathrm{H} 36 \mathrm{O} 4$ & 72.55 \\
\hline & $1000336-60-4^{a}$ & 44515574 & i-Propyl 12-methyl-tridecanoate & 270.26 & $\mathrm{C} 17 \mathrm{H} 34 \mathrm{O} 2$ & 73.73 \\
\hline & $1000374-06-1^{a}$ & 3023652 & 1,7-di-iso-propylnaphthalene & 212.16 & $\mathrm{C} 16 \mathrm{H} 20$ & 79.77 \\
\hline & $101-86-0$ & 7585 & Octanal, 2-(phenylmethylene)- & 216.15 & $\mathrm{C} 15 \mathrm{H} 20 \mathrm{O}$ & 85.79 \\
\hline & 105794-58-9 & 537071 & 1-Heptatriacotanol & 536.59 & $\mathrm{C} 37 \mathrm{H} 76 \mathrm{O}$ & 70.03 \\
\hline & 13466-78-9 & 26049 & 3-Carene & 136.13 & $\mathrm{C} 10 \mathrm{H} 16$ & 92.31 \\
\hline & $16507-61-2$ & 5367784 & cis-1-Chloro-9-octadecene & 286.24 & $\mathrm{C} 18 \mathrm{H} 35 \mathrm{Cl}$ & 74.15 \\
\hline & $242794-76-9$ & 564746 & Bicyclo[5.2.0]nonane, 2-methylene-4,8,8-trimethyl-4-vinyl- & 204.19 & $\mathrm{C} 15 \mathrm{H} 24$ & 85.25 \\
\hline & 294-62-2 & 9268 & Cyclododecane & 168.19 & $\mathrm{C} 12 \mathrm{H} 24$ & 74.42 \\
\hline & $499-97-8$ & 68140 & Cyclohexane, 1-methylene-4-(1-methylethenyl)- & 136.13 & $\mathrm{C} 10 \mathrm{H} 16$ & 88.01 \\
\hline & $535-77-3$ & 10812 & Benzene, 1-methyl-3-(1-methylethyl)- & 134.11 & $\mathrm{C} 10 \mathrm{H} 14$ & 84.43 \\
\hline & $546-80-5$ & 261491 & Bicyclo[3.1.0]hex-2-ene, 2-methyl-5-(1-methylethyl)- & 136.13 & $\mathrm{C} 10 \mathrm{H} 16$ & 89.31 \\
\hline & $586-63-0$ & 102443 & Cyclohexene, 3-methyl-6-(1-methylethylidene)- & 136.13 & $\mathrm{C} 10 \mathrm{H} 16$ & 85.52 \\
\hline & $5989-27-5$ & 440917 & D-Limonene & 136.13 & $\mathrm{C} 10 \mathrm{H} 16$ & 91.24 \\
\hline \multirow[t]{12}{*}{ LDPE 1} & $1000333-58-3^{a}$ & 12541027 & cis-13-Octadecenoic acid, methyl ester & 296.27 & $\mathrm{C} 19 \mathrm{H} 36 \mathrm{O} 2$ & 75.47 \\
\hline & $1000336-50-5^{a}$ & 13908974 & Methyl 9-eicosenoate & 324.30 & $\mathrm{C} 21 \mathrm{H} 40 \mathrm{O} 2$ & 72.97 \\
\hline & $1000364-37-1^{a}$ & 91738699 & 2-Methyl-3,5-dinitrobenzyl alcohol, TBDMS derivative & 326.13 & $\mathrm{C} 14 \mathrm{H} 22 \mathrm{~N} 2 \mathrm{O} 5 \mathrm{Si}$ & 71.01 \\
\hline & $1000374-06-1^{a}$ & 3023652 & 1,7-di-iso-propylnaphthalene & 212.16 & $\mathrm{C} 16 \mathrm{H} 20$ & 74.02 \\
\hline & $1000374-17-9^{a}$ & 6428435 & 7-epi-cis-sesquisabinene hydrate & 222.20 & $\mathrm{C} 15 \mathrm{H} 26 \mathrm{O}$ & 72.79 \\
\hline & $10436-08-5$ & 547891 & cis-11-Eicosenamide & 309.30 & $\mathrm{C} 20 \mathrm{H} 39 \mathrm{NO}$ & 77.21 \\
\hline & $10436-09-6$ & 5365369 & trans-13-Docosenamide & 337.33 & $\mathrm{C} 22 \mathrm{H} 43 \mathrm{NO}$ & 84.90 \\
\hline & $10482-56-1$ & 443162 & L-.alpha.-Terpineol & 154.14 & $\mathrm{C} 10 \mathrm{H} 18 \mathrm{O}$ & 87.36 \\
\hline & 105794-58-9 & 537071 & 1-Heptatriacotanol & 536.59 & $\mathrm{C} 37 \mathrm{H} 76 \mathrm{O}$ & 70.34 \\
\hline & $1120-25-8$ & 643801 & 9-Hexadecenoic acid, methyl ester, (Z)- & 268.24 & $\mathrm{C} 17 \mathrm{H} 32 \mathrm{O} 2$ & 74.59 \\
\hline & $112-63-0$ & 5284421 & 9,12-Octadecadienoic acid (Z,Z)-, methyl ester & 294.26 & $\mathrm{C} 19 \mathrm{H} 34 \mathrm{O} 2$ & 89.17 \\
\hline & $112-80-1$ & 445639 & Oleic Acid & 282.26 & $\mathrm{C} 18 \mathrm{H} 34 \mathrm{O} 2$ & 70.26 \\
\hline
\end{tabular}




\begin{tabular}{|c|c|c|c|c|c|c|}
\hline \multirow[t]{14}{*}{ Sample } & CAS & $\begin{array}{l}\text { PubChem } \\
\text { CID }\end{array}$ & Name according to NIST & Mass & Formula & Score \\
\hline & $115-99-1$ & 61040 & 1,6-Octadien-3-ol, 3,7-dimethyl-, formate & 182.13 & $\mathrm{C} 11 \mathrm{H} 18 \mathrm{O} 2$ & 81.34 \\
\hline & $13466-78-9$ & 26049 & 3-Carene & 136.13 & $\mathrm{C} 10 \mathrm{H} 16$ & 82.42 \\
\hline & $1632-73-1$ & 15406 & Fenchol & 154.14 & $\mathrm{C} 10 \mathrm{H} 18 \mathrm{O}$ & 80.75 \\
\hline & $1732-10-1$ & 15612 & Nonanedioic acid, dimethyl ester & 216.14 & $\mathrm{C} 11 \mathrm{H} 20 \mathrm{O} 4$ & 76.33 \\
\hline & $17367-08-7$ & 549041 & Ethanol, 2-(9,12-octadecadienyloxy)-, (Z,Z)- & 310.29 & $\mathrm{C} 20 \mathrm{H} 38 \mathrm{O} 2$ & 71.76 \\
\hline & 2091-29-4 & 4668 & 9-Hexadecenoic acid & 254.23 & $\mathrm{C} 16 \mathrm{H} 30 \mathrm{O} 2$ & 72.15 \\
\hline & $562-74-3$ & 11230 & Terpinen-4-ol & 154.14 & $\mathrm{C} 10 \mathrm{H} 18 \mathrm{O}$ & 82.74 \\
\hline & $56554-30-4$ & 556196 & 7,10,13-Hexadecatrienoic acid, methyl ester & 264.21 & $\mathrm{C} 17 \mathrm{H} 28 \mathrm{O} 2$ & 83.82 \\
\hline & $56630-69-4$ & 61268 & 13-Docosenoic acid, methyl ester & 352.33 & $\mathrm{C} 23 \mathrm{H} 44 \mathrm{O} 2$ & 90.77 \\
\hline & $57156-97-5$ & 5365571 & 12,15-Octadecadienoic acid, methyl ester & 294.26 & $\mathrm{C} 19 \mathrm{H} 34 \mathrm{O} 2$ & 89.69 \\
\hline & $5989-27-5$ & 440917 & D-Limonene & 136.13 & $\mathrm{C} 10 \mathrm{H} 16$ & 82.93 \\
\hline & $60-33-3$ & 5280450 & 9,12-Octadecadienoic acid (Z,Z)- & 280.24 & $\mathrm{C} 18 \mathrm{H} 32 \mathrm{O} 2$ & 72.77 \\
\hline & $81601-03-8$ & 91694967 & Geranyl oleate & 418.38 & $\mathrm{C} 28 \mathrm{H} 50 \mathrm{O} 2$ & 75.52 \\
\hline \multirow[t]{7}{*}{ LDPE 2} & $112-62-9$ & 5364509 & 9-Octadecenoic acid (Z)-, methyl ester & 296.27 & $\mathrm{C} 19 \mathrm{H} 36 \mathrm{O} 2$ & 87.08 \\
\hline & $5129-56-6$ & 554144 & Undecanoic acid, 10-methyl-, methyl ester & 214.19 & $\mathrm{C} 13 \mathrm{H} 26 \mathrm{O} 2$ & 83.16 \\
\hline & $5129-58-8$ & 21204 & Tridecanoic acid, 12-methyl-, methyl ester & 242.23 & $\mathrm{C} 15 \mathrm{H} 30 \mathrm{O} 2$ & 73.08 \\
\hline & $5129-61-3$ & 110444 & Heptadecanoic acid, 16-methyl-, methyl ester & 298.29 & $\mathrm{C} 19 \mathrm{H} 38 \mathrm{O} 2$ & 74.37 \\
\hline & $57156-97-5$ & 5365571 & 12,15-Octadecadienoic acid, methyl ester & 294.26 & $\mathrm{C} 19 \mathrm{H} 34 \mathrm{O} 2$ & 88.65 \\
\hline & $6386-38-5$ & 62603 & Benzenepropanoic acid, 3,5-bis(1,1-dimethylethyl)-4-hydroxy-, methyl ester & 292.20 & $\mathrm{C} 18 \mathrm{H} 28 \mathrm{O} 3$ & 73.29 \\
\hline & $96-76-4$ & 7311 & 2,4-Di-tert-butylphenol & 206.17 & $\mathrm{C} 14 \mathrm{H} 22 \mathrm{O}$ & 82.88 \\
\hline \multirow[t]{3}{*}{ LDPE 3} & $6386-38-5$ & 62603 & Benzenepropanoic acid, 3,5-bis(1,1-dimethylethyl)-4-hydroxy-, methyl ester & 292.20 & $\mathrm{C} 18 \mathrm{H} 28 \mathrm{O} 3$ & 89.02 \\
\hline & $82304-66-3$ & 545303 & 7,9-Di-tert-butyl-1-oxaspiro(4,5)deca-6,9-diene-2,8-dione & 276.17 & $\mathrm{C} 17 \mathrm{H} 24 \mathrm{O} 3$ & 82.82 \\
\hline & $96-76-4$ & 7311 & 2,4-Di-tert-butylphenol & 206.17 & $\mathrm{C} 14 \mathrm{H} 22 \mathrm{O}$ & 81.84 \\
\hline \multirow[t]{14}{*}{ LDPE 4} & $1000131-11-6^{a}$ & 5362676 & Z,Z,Z-1,4,6,9-Nonadecatetraene & 260.25 & $\mathrm{C} 19 \mathrm{H} 32$ & 73.26 \\
\hline & $1000131-33-2^{a}$ & 5363633 & Z-(13,14-Epoxy)tetradec-11-en-1-ol acetate & 268.20 & $\mathrm{C} 16 \mathrm{H} 28 \mathrm{O} 3$ & 70.02 \\
\hline & $1000142-34-3^{a}$ & 591964 & 2-Adamantanol, 2-(bromomethyl)- & 244.05 & $\mathrm{C} 11 \mathrm{H} 17 \mathrm{BrO}$ & 73.95 \\
\hline & $1000192-65-0^{a}$ & 543423 & 1,2-15,16-Diepoxyhexadecane & 254.23 & $\mathrm{C} 16 \mathrm{H} 30 \mathrm{O} 2$ & 73.92 \\
\hline & $1000314-35-6^{a}$ & 6421281 & (E,Z,Z)-2,4,7-Tridecatrienal & 192.15 & $\mathrm{C} 13 \mathrm{H} 20 \mathrm{O}$ & 70.48 \\
\hline & $1000336-36-7^{a}$ & 14122946 & Methyl 4,7,10,13-hexadecatetraenoate & 262.19 & $\mathrm{C} 17 \mathrm{H} 26 \mathrm{O} 2$ & 72.54 \\
\hline & $1000336-38-4^{a}$ & 91694372 & Methyl 3-cis,9-cis,12-cis-octadecatrienoate & 292.24 & $\mathrm{C} 19 \mathrm{H} 32 \mathrm{O} 2$ & 71.26 \\
\hline & $1000336-60-4^{a}$ & 44515574 & i-Propyl 12-methyl-tridecanoate & 270.26 & $\mathrm{C} 17 \mathrm{H} 34 \mathrm{O} 2$ & 73.43 \\
\hline & $1000352-68-4^{a}$ & 88368751 & Oleyl alcohol, trifluoroacetate & 364.26 & $\mathrm{C} 20 \mathrm{H} 35 \mathrm{~F} 3 \mathrm{O} 2$ & 75.37 \\
\hline & $1000374-06-1^{a}$ & 3023652 & 1,7-di-iso-propylnaphthalene & 212.16 & $\mathrm{C} 16 \mathrm{H} 20$ & 79.77 \\
\hline & $1000374-18-0^{a}$ & 11972555 & .alpha.-acorenol & 222.20 & $\mathrm{C} 15 \mathrm{H} 26 \mathrm{O}$ & 86.08 \\
\hline & $1000405-59-5^{a}$ & 91701181 & Fumaric acid, 2-octyl tridec-2-yn-1-yl ester & 406.31 & $\mathrm{C} 25 \mathrm{H} 42 \mathrm{O} 4$ & 71.10 \\
\hline & $1000406-96-9^{a}$ & 91697642 & Undec-10-ynoic acid, tridec-2-yn-1-yl ester & 360.30 & $\mathrm{C} 24 \mathrm{H} 40 \mathrm{O} 2$ & 74.05 \\
\hline & $1000414-43-3^{a}$ & 91694966 & Geranyl palmitoleate & 390.35 & $\mathrm{C} 26 \mathrm{H} 46 \mathrm{O} 2$ & 75.56 \\
\hline
\end{tabular}




\begin{tabular}{|c|c|c|c|c|c|c|}
\hline Sample & CAS & $\begin{array}{l}\text { PubChem } \\
\text { CID }\end{array}$ & Name according to NIST & Mass & Formula & Score \\
\hline & $10287-53-3$ & 25127 & Parbenate & 193.11 & $\mathrm{C} 11 \mathrm{H} 15 \mathrm{NO} 2$ & 83.57 \\
\hline & $10482-56-1$ & 443162 & L-.alpha.-Terpineol & 154.14 & $\mathrm{C} 10 \mathrm{H} 18 \mathrm{O}$ & 87.52 \\
\hline & 105794-58-9 & 537071 & 1-Heptatriacotanol & 536.59 & $\mathrm{C} 37 \mathrm{H} 76 \mathrm{O}$ & 78.17 \\
\hline & $115-99-1$ & 61040 & 1,6-Octadien-3-ol, 3,7-dimethyl-, formate & 182.13 & $\mathrm{C} 11 \mathrm{H} 18 \mathrm{O} 2$ & 83.17 \\
\hline & 117066-77-0 & none & 2-((2S,4aR)-4a,8-Dimethyl-1,2,3,4,4a,5,6,7-octahydronaphthalen-2-yl)propan-2-ol & 222.20 & $\mathrm{C} 15 \mathrm{H} 26 \mathrm{O}$ & 85.50 \\
\hline & $120-51-4$ & 2345 & Benzyl Benzoate & 212.08 & $\mathrm{C} 14 \mathrm{H} 12 \mathrm{O} 2$ & 70.07 \\
\hline & $1209-71-8$ & 6432005 & $\begin{array}{l}\text { 2-Naphthalenemethanol, 1,2,3,4,4a,5,6,7-octahydro-.alpha.,.alpha.,4a,8-tetramethyl-, } \\
\text { (2R-cis)- }\end{array}$ & 222.20 & $\mathrm{C} 15 \mathrm{H} 26 \mathrm{O}$ & 87.40 \\
\hline & $13466-78-9$ & 26049 & 3-Carene & 136.13 & $\mathrm{C} 10 \mathrm{H} 16$ & 89.92 \\
\hline & $177205-54-8$ & 622707 & 1,2,3,4-Tetrahydro-1,4-ethanoanthracene, 9,10-dimethoxy- & 268.15 & $\mathrm{C} 18 \mathrm{H} 20 \mathrm{O} 2$ & 81.18 \\
\hline & 17735-94-3 & 5312518 & cis-13-Eicosenoic acid & 310.29 & $\mathrm{C} 20 \mathrm{H} 38 \mathrm{O} 2$ & 70.56 \\
\hline & $1845-30-3$ & 164888 & cis-Verbenol & 152.12 & $\mathrm{C} 10 \mathrm{H} 16 \mathrm{O}$ & 82.59 \\
\hline & $194607-96-0$ & 527206 & $\begin{array}{l}\text { 2-((4aS,8R,8aR)-4a,8-Dimethyl-3,4,4a,5,6,7,8,8a-octahydronaphthalen-2-yl)propan-2- } \\
\text { ol }\end{array}$ & 222.20 & $\mathrm{C} 15 \mathrm{H} 26 \mathrm{O}$ & 85.06 \\
\hline & $21747-46-6$ & 10910653 & $\begin{array}{l}\text { 1H-Cycloprop[e]azulene, 1a,2,3,5,6,7,7a,7b-octahydro-1,1,4,7-tetramethyl-, [1aR- } \\
\text { (1a.alpha.,7.alpha.,7a.beta.,7b.alpha.)]- }\end{array}$ & 204.19 & $\mathrm{C} 15 \mathrm{H} 24$ & 82.27 \\
\hline & $22117-09-5$ & 5367371 & 5,8,11-Heptadecatrien-1-ol & 250.23 & $\mathrm{C} 17 \mathrm{H} 30 \mathrm{O}$ & 72.18 \\
\hline & $2244-16-8$ & 16724 & D-Carvone & 150.10 & $\mathrm{C} 10 \mathrm{H} 14 \mathrm{O}$ & 78.01 \\
\hline & 29141-10-4 & 6553885 & (1R,2R,5S)-5-Methyl-2-(prop-1-en-2-yl)cyclohexanol & 154.14 & $\mathrm{C} 10 \mathrm{H} 18 \mathrm{O}$ & 80.60 \\
\hline & $294-62-2$ & 9268 & Cyclododecane & 168.19 & $\mathrm{C} 12 \mathrm{H} 24$ & 73.79 \\
\hline & $29803-82-5$ & 122485 & 2-Cyclohexen-1-ol, 1-methyl-4-(1-methylethyl)-, cis- & 154.14 & $\mathrm{C} 10 \mathrm{H} 18 \mathrm{O}$ & 70.29 \\
\hline & $353313^{a}$ & 17868 & Bicyclo[3.1.0]hex-2-ene, 2-methyl-5-(1-methylethyl)- & 136.13 & C10H16 & 87.39 \\
\hline & $463-40-1$ & 5280934 & 9,12,15-Octadecatrienoic acid, $(\mathrm{Z}, \mathrm{Z}, \mathrm{Z})$ - & 278.23 & $\mathrm{C} 18 \mathrm{H} 30 \mathrm{O} 2$ & 73.20 \\
\hline & $470-40-6$ & 11401461 & cis-Thujopsene & 204.19 & $\mathrm{C} 15 \mathrm{H} 24$ & 79.33 \\
\hline & $499-97-8$ & 68140 & Cyclohexane, 1-methylene-4-(1-methylethenyl)- & 136.13 & $\mathrm{C} 10 \mathrm{H} 16$ & 89.08 \\
\hline & $506-26-3$ & 5280933 & Gamolenic acid & 278.23 & $\mathrm{C} 18 \mathrm{H} 30 \mathrm{O} 2$ & 73.07 \\
\hline & $514-95-4$ & 578237 & 1,5,5-Trimethyl-6-methylene-cyclohexene & 136.13 & $\mathrm{C} 10 \mathrm{H} 16$ & 76.22 \\
\hline & $535-77-3$ & 10812 & Benzene, 1-methyl-3-(1-methylethyl)- & 134.11 & $\mathrm{C} 10 \mathrm{H} 14$ & 84.97 \\
\hline & $5392-40-5$ & 8843 & Citral & 152.12 & $\mathrm{C} 10 \mathrm{H} 16 \mathrm{O}$ & 80.01 \\
\hline & $562-74-3$ & 11230 & Terpinen-4-ol & 154.14 & $\mathrm{C} 10 \mathrm{H} 18 \mathrm{O}$ & 83.75 \\
\hline & $56666-38-7$ & 41961 & 2H-Pyran, tetrahydro-2-(12-pentadecynyloxy)- & 308.27 & $\mathrm{C} 20 \mathrm{H} 36 \mathrm{O} 2$ & 72.69 \\
\hline & $584-79-2$ & 11442 & Bioallethrin & 302.19 & $\mathrm{C} 19 \mathrm{H} 26 \mathrm{O} 3$ & 71.11 \\
\hline & $5989-27-5$ & 440917 & D-Limonene & 136.13 & $\mathrm{C} 10 \mathrm{H} 16$ & 83.74 \\
\hline & $61465-23-4$ & 577045 & (+-)-trans-1-Isopropenyl-4-methyl-1,4-cyclohexanediol & 170.13 & $\mathrm{C} 10 \mathrm{H} 18 \mathrm{O} 2$ & 71.12 \\
\hline & $7212-40-0$ & 12618691 & 2-Cyclohexen-1-ol, 1-methyl-4-(1-methylethenyl)-, trans- & 152.12 & $\mathrm{C} 10 \mathrm{H} 16 \mathrm{O}$ & 72.18 \\
\hline & $7452-79-1$ & 24020 & Butanoic acid, 2-methyl-, ethyl ester & 130.10 & $\mathrm{C} 7 \mathrm{H} 14 \mathrm{O} 2$ & 76.76 \\
\hline & $77-53-2$ & 65575 & Cedrol & 222.20 & $\mathrm{C} 15 \mathrm{H} 26 \mathrm{O}$ & 90.06 \\
\hline & $7785-70-8$ & 82227 & (1R)-2,6,6-Trimethylbicyclo[3.1.1]hept-2-ene & 136.13 & $\mathrm{C} 10 \mathrm{H} 16$ & 82.07 \\
\hline
\end{tabular}




\begin{tabular}{|c|c|c|c|c|c|c|}
\hline Sample & CAS & $\begin{array}{l}\text { PubChem } \\
\text { CID }\end{array}$ & Name according to NIST & Mass & Formula & Score \\
\hline & $7786-67-6$ & 24585 & Cyclohexanol, 5-methyl-2-(1-methylethenyl)- & 154.14 & $\mathrm{C} 10 \mathrm{H} 18 \mathrm{O}$ & 81.53 \\
\hline & $99-87-6$ & 7463 & p-Cymene & 134.11 & $\mathrm{C} 10 \mathrm{H} 14$ & 84.23 \\
\hline \multirow[t]{5}{*}{ PS 1} & $100-42-5$ & 7501 & Styrene & 104.06 & $\mathrm{C} 8 \mathrm{H} 8$ & 92.48 \\
\hline & 131758-71-9 & 562543 & (2,3-Diphenylcyclopropyl)methyl phenyl sulfoxide, trans- & 332.12 & $\mathrm{C} 22 \mathrm{H} 20 \mathrm{OS}$ & 76.37 \\
\hline & 20071-09-4 & 11954175 & Benzene, 1,1'-(1,2-cyclobutanediyl)bis-, trans- & 208.13 & C16H16 & 83.78 \\
\hline & $25558-23-0$ & 568889 & Cyclobutane, 1,3-diphenyl-, trans- & 208.13 & $\mathrm{C} 16 \mathrm{H} 16$ & 71.07 \\
\hline & $538-81-8$ & 641683 & 1,3-Butadiene, 1,4-diphenyl-, (E,E)- & 206.11 & $\mathrm{C} 16 \mathrm{H} 14$ & 78.93 \\
\hline \multirow[t]{10}{*}{ PS 2} & $1000192-89-2^{a}$ & 5375831 & Thiocarbamic acid, N,N-dimethyl, S-1,3-diphenyl-2-butenyl ester & 311.13 & $\mathrm{C} 19 \mathrm{H} 21 \mathrm{NOS}$ & 72.30 \\
\hline & $1000336-60-4^{a}$ & 44515574 & i-Propyl 12-methyl-tridecanoate & 270.26 & $\mathrm{C} 17 \mathrm{H} 34 \mathrm{O} 2$ & 81.81 \\
\hline & $100-42-5$ & 7501 & Styrene & 104.06 & $\mathrm{C} 8 \mathrm{H} 8$ & 86.69 \\
\hline & $10436-08-5$ & 547891 & cis-11-Eicosenamide & 309.30 & $\mathrm{C} 20 \mathrm{H} 39 \mathrm{NO}$ & 75.46 \\
\hline & $120-51-4$ & 2345 & Benzyl Benzoate & 212.08 & $\mathrm{C} 14 \mathrm{H} 12 \mathrm{O} 2$ & 73.31 \\
\hline & 20071-09-4 & 11954175 & Benzene, 1,1'-(1,2-cyclobutanediyl)bis-, trans- & 208.13 & C16H16 & 74.04 \\
\hline & $23470-00-0$ & 123409 & Hexadecanoic acid, 2-hydroxy-1-(hydroxymethyl)ethyl ester & 330.28 & $\mathrm{C} 19 \mathrm{H} 38 \mathrm{O} 4$ & 81.59 \\
\hline & 29422-13-7 & 34581 & Naphthalene, 1,2,3,4-tetrahydro-2-phenyl- & 208.13 & $\mathrm{C} 16 \mathrm{H} 16$ & 72.68 \\
\hline & $301-02-0$ & 5283387 & 9-Octadecenamide, $(Z)-$ & 281.27 & $\mathrm{C} 18 \mathrm{H} 35 \mathrm{NO}$ & 87.13 \\
\hline & $56728-02-0$ & 609923 & Benzene, 1,1'-[2-methyl-2-(phenylthio)cyclopropylidene]bis- & 316.13 & $\mathrm{C} 22 \mathrm{H} 20 \mathrm{~S}$ & 70.10 \\
\hline \multirow[t]{5}{*}{ PS 3} & $100-42-5$ & 7501 & Styrene & 104.06 & C8H8 & 89.70 \\
\hline & 131758-71-9 & 562543 & (2,3-Diphenylcyclopropyl)methyl phenyl sulfoxide, trans- & 332.12 & $\mathrm{C} 22 \mathrm{H} 20 \mathrm{OS}$ & 76.42 \\
\hline & 20071-09-4 & 11954175 & Benzene, 1,1'-(1,2-cyclobutanediyl)bis-, trans- & 208.13 & $\mathrm{C} 16 \mathrm{H} 16$ & 86.62 \\
\hline & $538-81-8$ & 641683 & 1,3-Butadiene, 1,4-diphenyl-, (E,E)- & 206.11 & $\mathrm{C} 16 \mathrm{H} 14$ & 80.62 \\
\hline & $56728-02-0$ & 609923 & Benzene, 1,1'-[2-methyl-2-(phenylthio)cyclopropylidene]bis- & 316.13 & $\mathrm{C} 22 \mathrm{H} 20 \mathrm{~S}$ & 77.61 \\
\hline \multirow[t]{14}{*}{ PS 4} & $1000130-80-7^{a}$ & 5363617 & E-11-Methyl-12-tetradecen-1-ol acetate & 268.24 & $\mathrm{C} 17 \mathrm{H} 32 \mathrm{O} 2$ & 77.16 \\
\hline & $1000130-81-0^{a}$ & 549821 & 11,13-Dimethyl-12-tetradecen-1-ol acetate & 282.26 & $\mathrm{C} 18 \mathrm{H} 34 \mathrm{O} 2$ & 78.57 \\
\hline & $1000192-65-0^{a}$ & 543423 & 1,2-15,16-Diepoxyhexadecane & 254.23 & $\mathrm{C} 16 \mathrm{H} 30 \mathrm{O} 2$ & 73.64 \\
\hline & $1000374-06-1^{a}$ & 3023652 & 1,7-di-iso-propylnaphthalene & 212.16 & $\mathrm{C} 16 \mathrm{H} 20$ & 72.97 \\
\hline & $1000382-54-3^{a}$ & 91693137 & Carbonic acid, eicosyl vinyl ester & 368.33 & $\mathrm{C} 23 \mathrm{H} 44 \mathrm{O} 3$ & 75.68 \\
\hline & $1000406-16-9^{a}$ & 91692473 & Undec-10-ynoic acid, hexadecyl ester & 406.38 & $\mathrm{C} 27 \mathrm{H} 50 \mathrm{O} 2$ & 75.59 \\
\hline & $100-41-4$ & 7500 & Ethylbenzene & 106.08 & $\mathrm{C} 8 \mathrm{H} 10$ & 89.77 \\
\hline & $100-42-5$ & 7501 & Styrene & 104.06 & $\mathrm{C} 8 \mathrm{H} 8$ & 89.77 \\
\hline & $150-86-7$ & 5280435 & Phytol & 296.31 & $\mathrm{C} 20 \mathrm{H} 40 \mathrm{O}$ & 77.15 \\
\hline & $17634-51-4$ & 561243 & 1,3,5-Cycloheptatriene, 7-ethyl- & 120.09 & C9H12 & 72.65 \\
\hline & 20071-09-4 & 11954175 & Benzene, 1,1'-(1,2-cyclobutanediyl)bis-, trans- & 208.13 & $\mathrm{C} 16 \mathrm{H} 16$ & 86.94 \\
\hline & $56728-02-0$ & 609923 & Benzene, 1,1'-[2-methyl-2-(phenylthio)cyclopropylidene]bis- & 316.13 & $\mathrm{C} 22 \mathrm{H} 20 \mathrm{~S}$ & 80.56 \\
\hline & $5989-27-5$ & 440917 & D-Limonene & 136.13 & $\mathrm{C} 10 \mathrm{H} 16$ & 78.77 \\
\hline & $98-82-8$ & 7406 & Benzene, (1-methylethyl)- & 120.09 & $\mathrm{C} 9 \mathrm{H} 12$ & 81.26 \\
\hline PP 1 & n.d. & & & & & \\
\hline
\end{tabular}




\begin{tabular}{|c|c|c|c|c|c|c|}
\hline Sample & CAS & $\begin{array}{l}\text { PubChem } \\
\text { CID }\end{array}$ & Name according to NIST & Mass & Formula & Score \\
\hline PP 2 & n.d. & & & & & \\
\hline \multirow[t]{16}{*}{ PP 3} & $1000336-43-6^{a}$ & 20619411 & Methyl 8-methyl-nonanoate & 186.16 & $\mathrm{C} 11 \mathrm{H} 22 \mathrm{O} 2$ & 77.31 \\
\hline & $1000339-14-5^{a}$ & 91695412 & Fumaric acid, 2-ethylhexyl undecyl ester & 382.31 & $\mathrm{C} 23 \mathrm{H} 42 \mathrm{O} 4$ & 70.28 \\
\hline & $1000368-53-5^{a}$ & 91205583 & Ethyl stearate, 9,12-diepoxy & 340.26 & $\mathrm{C} 20 \mathrm{H} 36 \mathrm{O} 4$ & 72.53 \\
\hline & $1000381-53-1^{a}$ & 91726212 & Succinic acid, 2-(2-chlorophenoxy)ethyl ethyl ester & 300.08 & $\mathrm{C} 14 \mathrm{H} 17 \mathrm{ClO} 5$ & 71.07 \\
\hline & $109-43-3$ & 7986 & Decanedioic acid, dibutyl ester & 314.25 & $\mathrm{C} 18 \mathrm{H} 34 \mathrm{O} 4$ & 82.40 \\
\hline & $111-11-5$ & 8091 & Octanoic acid, methyl ester & 158.13 & C9H18O2 & 82.15 \\
\hline & $128-37-0$ & 31404 & Butylated Hydroxytoluene & 220.18 & $\mathrm{C} 15 \mathrm{H} 24 \mathrm{O}$ & 87.82 \\
\hline & $24560-98-3$ & 119250 & Oxiraneoctanoic acid, 3-octyl-, cis- & 298.25 & $\mathrm{C} 18 \mathrm{H} 34 \mathrm{O} 3$ & 71.24 \\
\hline & $33368-86-4$ & 10186592 & 2-(Octanoyloxy)propane-1,3-diyl bis(decanoate) & 526.42 & $\mathrm{C} 31 \mathrm{H} 58 \mathrm{O} 6$ & 73.10 \\
\hline & $33368-87-5$ & 10436013 & 2-(Decanoyloxy)propane-1,3-diyl dioctanoate & 498.39 & C29H54O6 & 73.20 \\
\hline & 4098-71-9 & 169132 & Isophorone diisocyanate & 222.14 & $\mathrm{C} 12 \mathrm{H} 18 \mathrm{~N} 2 \mathrm{O} 2$ & 80.96 \\
\hline & $5129-61-3$ & 110444 & Heptadecanoic acid, 16-methyl-, methyl ester & 298.29 & $\mathrm{C} 19 \mathrm{H} 38 \mathrm{O} 2$ & 80.72 \\
\hline & $628-97-7$ & 12366 & Hexadecanoic acid, ethyl ester & 284.27 & $\mathrm{C} 18 \mathrm{H} 36 \mathrm{O} 2$ & 75.72 \\
\hline & $6386-38-5$ & 62603 & Benzenepropanoic acid, 3,5-bis(1,1-dimethylethyl)-4-hydroxy-, methyl ester & 292.20 & $\mathrm{C} 18 \mathrm{H} 28 \mathrm{O} 3$ & 74.23 \\
\hline & $77-90-7$ & 6505 & Tributyl acetylcitrate & 402.23 & $\mathrm{C} 20 \mathrm{H} 34 \mathrm{O} 8$ & 76.77 \\
\hline & $87-97-8$ & 6911 & Phenol, 2,6-bis(1,1-dimethylethyl)-4-(methoxymethyl)- & 250.19 & $\mathrm{C} 16 \mathrm{H} 26 \mathrm{O} 2$ & 77.23 \\
\hline \multirow[t]{5}{*}{ PP 4} & $1000336-62-4^{a}$ & 53745103 & i-Propyl 14-methyl-pentadecanoate & 298.29 & $\mathrm{C} 19 \mathrm{H} 38 \mathrm{O} 2$ & 79.06 \\
\hline & $101-68-8$ & 7570 & Benzene, 1,1'-methylenebis[4-isocyanato- & 250.07 & $\mathrm{C} 15 \mathrm{H} 10 \mathrm{~N} 2 \mathrm{O} 2$ & 90.58 \\
\hline & $10436-09-6$ & 5365369 & trans-13-Docosenamide & 337.33 & $\mathrm{C} 22 \mathrm{H} 43 \mathrm{NO}$ & 72.31 \\
\hline & $301-02-0$ & 5283387 & 9-Octadecenamide, $(\mathrm{Z})$ - & 281.27 & $\mathrm{C} 18 \mathrm{H} 35 \mathrm{NO}$ & 85.30 \\
\hline & $77-90-7$ & 6505 & Tributyl acetylcitrate & 402.23 & $\mathrm{C} 20 \mathrm{H} 34 \mathrm{O} 8$ & 84.50 \\
\hline \multirow[t]{15}{*}{ PP 5} & $1000333-58-3^{a}$ & 12541027 & cis-13-Octadecenoic acid, methyl ester & 296.27 & $\mathrm{C} 19 \mathrm{H} 36 \mathrm{O} 2$ & 74.59 \\
\hline & $1000336-60-4^{a}$ & 44515574 & i-Propyl 12-methyl-tridecanoate & 270.26 & $\mathrm{C} 17 \mathrm{H} 34 \mathrm{O} 2$ & 86.61 \\
\hline & $1000351-75-2^{a}$ & 14574254 & Eicosyl trifluoroacetate & 394.31 & $\mathrm{C} 22 \mathrm{H} 41 \mathrm{~F} 3 \mathrm{O} 2$ & 80.57 \\
\hline & $1000368-56-5^{a}$ & 91691599 & 2-Hexyldodecyl isobutyrate & 340.33 & $\mathrm{C} 22 \mathrm{H} 44 \mathrm{O} 2$ & 80.81 \\
\hline & $1000374-06-1^{a}$ & 3023652 & 1,7-di-iso-propylnaphthalene & 212.16 & $\mathrm{C} 16 \mathrm{H} 20$ & 71.86 \\
\hline & $1000382-54-3^{a}$ & 91693137 & Carbonic acid, eicosyl vinyl ester & 368.33 & $\mathrm{C} 23 \mathrm{H} 44 \mathrm{O} 3$ & 70.61 \\
\hline & $103-95-7$ & 517827 & 3-(4-Isopropylphenyl)-2-methylpropionaldehyde & 190.14 & $\mathrm{C} 13 \mathrm{H} 18 \mathrm{O}$ & 87.10 \\
\hline & $112-39-0$ & 8181 & Hexadecanoic acid, methyl ester & 270.26 & $\mathrm{C} 17 \mathrm{H} 34 \mathrm{O} 2$ & 85.30 \\
\hline & $119-61-9$ & 3102 & Benzophenone & 182.07 & $\mathrm{C} 13 \mathrm{H} 10 \mathrm{O}$ & 82.23 \\
\hline & $1222-05-5$ & 91497 & Cyclopenta[g]-2-benzopyran, 1,3,4,6,7,8-hexahydro-4,6,6,7,8,8-hexamethyl- & 258.20 & $\mathrm{C} 18 \mathrm{H} 26 \mathrm{O}$ & 94.81 \\
\hline & $128-37-0$ & 31404 & Butylated Hydroxytoluene & 220.18 & $\mathrm{C} 15 \mathrm{H} 24 \mathrm{O}$ & 89.19 \\
\hline & $13491-79-7$ & 26068 & Cyclohexanol, 2-(1,1-dimethylethyl)- & 156.15 & $\mathrm{C} 10 \mathrm{H} 20 \mathrm{O}$ & 78.36 \\
\hline & $2425-77-6$ & 95337 & 1-Decanol, 2-hexyl- & 242.26 & $\mathrm{C} 16 \mathrm{H} 34 \mathrm{O}$ & 79.62 \\
\hline & $5129-56-6$ & 554144 & Undecanoic acid, 10-methyl-, methyl ester & 214.19 & $\mathrm{C} 13 \mathrm{H} 26 \mathrm{O} 2$ & 76.80 \\
\hline & $5129-58-8$ & 21204 & Tridecanoic acid, 12-methyl-, methyl ester & 242.23 & $\mathrm{C} 15 \mathrm{H} 30 \mathrm{O} 2$ & 79.44 \\
\hline
\end{tabular}




\begin{tabular}{|c|c|c|c|c|c|c|}
\hline Sample & CAS & $\begin{array}{l}\text { PubChem } \\
\text { CID }\end{array}$ & Name according to NIST & Mass & Formula & Score \\
\hline & $5129-61-3$ & 110444 & Heptadecanoic acid, 16-methyl-, methyl ester & 298.29 & $\mathrm{C} 19 \mathrm{H} 38 \mathrm{O} 2$ & 89.70 \\
\hline & $5348-82-3$ & 79299 & Acetic acid, chloro-, octadecyl ester & 346.26 & $\mathrm{C} 20 \mathrm{H} 39 \mathrm{ClO} 2$ & 79.89 \\
\hline & $55741-10-1$ & 615306 & Naphthalene, 6,7-diethyl-1,2,3,4-tetrahydro-1,1,4,4-tetramethyl- & 244.22 & $\mathrm{C} 18 \mathrm{H} 28$ & 78.47 \\
\hline & $6386-38-5$ & 62603 & Benzenepropanoic acid, 3,5-bis(1,1-dimethylethyl)-4-hydroxy-, methyl ester & 292.20 & $\mathrm{C} 18 \mathrm{H} 28 \mathrm{O} 3$ & 76.72 \\
\hline & $7460-74-4$ & 81964 & Pentanoic acid, 2-phenylethyl ester & 206.13 & $\mathrm{C} 13 \mathrm{H} 18 \mathrm{O} 2$ & 75.64 \\
\hline & $80-54-6$ & 228987 & Lilial & 204.15 & $\mathrm{C} 14 \mathrm{H} 20 \mathrm{O}$ & 81.43 \\
\hline & $88-29-9$ & 6930 & 7-Acetyl-6-ethyl-1,1,4,4-tetramethyltetralin & 258.20 & $\mathrm{C} 18 \mathrm{H} 26 \mathrm{O}$ & 78.53 \\
\hline PET 1 & $5989-27-5$ & 440917 & D-Limonene & 136.13 & $\mathrm{C} 10 \mathrm{H} 16$ & 86.85 \\
\hline PET 2 & n.d. & & & & & \\
\hline PET 3 & n.d. & & & & & \\
\hline PET 4 & n.d. & & & & & \\
\hline PET 5 & n.d. & & & & & \\
\hline \multirow[t]{16}{*}{ PVC 1} & $1000043-05-3^{a}$ & 6452096 & Ethyl iso-allocholate & 436.32 & $\mathrm{C} 26 \mathrm{H} 44 \mathrm{O} 5$ & 70.14 \\
\hline & $1000368-53-5^{a}$ & 91205583 & Ethyl stearate, 9,12-diepoxy & 340.26 & $\mathrm{C} 20 \mathrm{H} 36 \mathrm{O} 4$ & 82.01 \\
\hline & $1000383-37-7^{a}$ & 5354568 & Glycidyl oleate & 338.28 & $\mathrm{C} 21 \mathrm{H} 38 \mathrm{O} 3$ & 75.46 \\
\hline & $111-03-5$ & 5283468 & 9-Octadecenoic acid (Z)-, 2,3-dihydroxypropyl ester & 356.29 & $\mathrm{C} 21 \mathrm{H} 40 \mathrm{O} 4$ & 70.61 \\
\hline & $119-61-9$ & 3102 & Benzophenone & 182.07 & $\mathrm{C} 13 \mathrm{H} 10 \mathrm{O}$ & 76.29 \\
\hline & $14290-23-4$ & 539937 & Myristin, 1,3-diaceto-2- & 386.27 & $\mathrm{C} 21 \mathrm{H} 38 \mathrm{O} 6$ & 79.34 \\
\hline & $17598-94-6$ & 33979 & Dodecanoic acid, 1-(hydroxymethyl)-1,2-ethanediyl ester & 456.38 & $\mathrm{C} 27 \mathrm{H} 52 \mathrm{O} 5$ & 72.34 \\
\hline & $26719-54-0$ & 537376 & Dodecanoic acid 3-dodecanoyloxy-propyl ester & 440.39 & $\mathrm{C} 27 \mathrm{H} 52 \mathrm{O} 4$ & 71.92 \\
\hline & $4337-65-9$ & 20342 & Hexanedioic acid, mono(2-ethylhexyl)ester & 258.18 & $\mathrm{C} 14 \mathrm{H} 26 \mathrm{O} 4$ & 76.61 \\
\hline & $5129-56-6$ & 554144 & Undecanoic acid, 10-methyl-, methyl ester & 214.19 & $\mathrm{C} 13 \mathrm{H} 26 \mathrm{O} 2$ & 81.77 \\
\hline & $5129-61-3$ & 110444 & Heptadecanoic acid, 16-methyl-, methyl ester & 298.29 & $\mathrm{C} 19 \mathrm{H} 38 \mathrm{O} 2$ & 81.70 \\
\hline & $52380-33-3$ & 5364432 & 11-Octadecenoic acid, methyl ester & 296.27 & $\mathrm{C} 19 \mathrm{H} 36 \mathrm{O} 2$ & 82.24 \\
\hline & $55268-70-7$ & 191981 & Hexadecanoic acid, 2,3-bis(acetyloxy)propyl ester & 414.30 & $\mathrm{C} 23 \mathrm{H} 42 \mathrm{O} 6$ & 72.01 \\
\hline & $55429-68-0$ & 539928 & Eicosanoic acid, 2-(acetyloxy)-1-[(acetyloxy)methyl]ethyl ester & 470.36 & $\mathrm{C} 27 \mathrm{H} 50 \mathrm{O} 6$ & 73.40 \\
\hline & $645-66-9$ & 69527 & Lauric anhydride & 382.35 & $\mathrm{C} 24 \mathrm{H} 46 \mathrm{O} 3$ & 73.38 \\
\hline & $761-35-3$ & 99931 & Hexadecanoic acid, 1-(hydroxymethyl)-1,2-ethanediyl ester & 568.51 & $\mathrm{C} 35 \mathrm{H} 68 \mathrm{O} 5$ & 71.87 \\
\hline \multirow[t]{9}{*}{ PVC 2} & $1000324-52-2^{a}$ & 91718008 & Adipic acid, isohexyl methyl ester & 244.17 & $\mathrm{C} 13 \mathrm{H} 24 \mathrm{O} 4$ & 74.23 \\
\hline & $1000339-40-5^{a}$ & 12151622 & 1,2-Cyclohexanedicarboxylic acid, dinonyl ester & 424.36 & $\mathrm{C} 26 \mathrm{H} 48 \mathrm{O} 4$ & 80.59 \\
\hline & $1000339-74-3^{a}$ & 91721826 & 1,2-Cyclohexanedicarboxylic acid, cyclohexylmethyl nonyl ester & 394.31 & $\mathrm{C} 24 \mathrm{H} 42 \mathrm{O} 4$ & 78.08 \\
\hline & $1000339-85-1^{a}$ & 91721974 & 1,2-Cyclohexanedicarboxylic acid, 3,5-dimethylcyclohexyl nonyl ester & 408.32 & $\mathrm{C} 25 \mathrm{H} 44 \mathrm{O} 4$ & 75.36 \\
\hline & $109-39-7$ & 66959 & 2-Butoxyethyl oleate & 382.35 & $\mathrm{C} 24 \mathrm{H} 46 \mathrm{O} 3$ & 78.43 \\
\hline & $112-62-9$ & 5364509 & 9-Octadecenoic acid (Z)-, methyl ester & 296.27 & $\mathrm{C} 19 \mathrm{H} 36 \mathrm{O} 2$ & 80.99 \\
\hline & $117-81-7$ & 8343 & Bis(2-ethylhexyl) phthalate & 390.28 & $\mathrm{C} 24 \mathrm{H} 38 \mathrm{O} 4$ & 72.28 \\
\hline & $128-37-0$ & 31404 & Butylated Hydroxytoluene & 220.18 & $\mathrm{C} 15 \mathrm{H} 24 \mathrm{O}$ & 86.70 \\
\hline & 29761-21-5 & 34697 & Phosphoric acid, isodecyl diphenyl ester & 390.20 & $\mathrm{C} 22 \mathrm{H} 31 \mathrm{O} 4 \mathrm{P}$ & 74.21 \\
\hline
\end{tabular}




\begin{tabular}{|c|c|c|c|c|c|c|}
\hline Sample & CAS & $\begin{array}{l}\text { PubChem } \\
\text { CID }\end{array}$ & Name according to NIST & Mass & Formula & Score \\
\hline & $301-02-0$ & 5283387 & 9-Octadecenamide, (Z)- & 281.27 & $\mathrm{C} 18 \mathrm{H} 35 \mathrm{NO}$ & 81.87 \\
\hline & $4337-65-9$ & 20342 & Hexanedioic acid, mono(2-ethylhexyl)ester & 258.18 & $\mathrm{C} 14 \mathrm{H} 26 \mathrm{O} 4$ & 76.59 \\
\hline \multirow[t]{35}{*}{ PVC 3} & $1000115-60-4^{a}$ & 5369409 & 5-Hexadecenoic acid, 2-methoxy-, methyl ester & 298.25 & C18H34O3 & 71.33 \\
\hline & $1000127-49-8^{a}$ & 590850 & Phen-1,4-diol, 2,3-dimethyl-5-trifluoromethyl- & 206.06 & $\mathrm{C} 9 \mathrm{H} 9 \mathrm{~F} 3 \mathrm{O} 2$ & 72.01 \\
\hline & $1000131-33-2^{a}$ & 5363633 & Z-(13,14-Epoxy)tetradec-11-en-1-ol acetate & 268.20 & $\mathrm{C} 16 \mathrm{H} 28 \mathrm{O} 3$ & 71.95 \\
\hline & $1000215-67-6^{a}$ & none & trans-2,4-Dimethylthiane, S,S-dioxide & 162.07 & $\mathrm{C} 7 \mathrm{H} 14 \mathrm{O} 2 \mathrm{~S}$ & 73.76 \\
\hline & $1000215-75-3^{a}$ & none & trans-2-methyl-4-n-pentylthiane, S,S-dioxide & 218.13 & $\mathrm{C} 11 \mathrm{H} 22 \mathrm{O} 2 \mathrm{~S}$ & 82.26 \\
\hline & $1000253-26-1^{a}$ & 569846 & Octanediamide, N,N'-di-benzoyloxy- & 412.16 & $\mathrm{C} 22 \mathrm{H} 24 \mathrm{~N} 2 \mathrm{O} 6$ & 71.67 \\
\hline & $1000270-36-9^{a}$ & 569440 & Benzamide, N-(1,3-dihydro-2-oxo-4-isobenzofuryl)- & 253.07 & C15H11NO3 & 79.15 \\
\hline & $1000324-49-0^{a}$ & 91713297 & Adipic acid, 2-ethylhexyl tetradecyl ester & 454.40 & C28H54O4 & 70.35 \\
\hline & $1000333-54-0^{a}$ & 15717634 & 17-Octadecynoic acid, methyl ester & 294.26 & $\mathrm{C} 19 \mathrm{H} 34 \mathrm{O} 2$ & 70.63 \\
\hline & $1000333-58-3^{a}$ & 12541027 & cis-13-Octadecenoic acid, methyl ester & 296.27 & $\mathrm{C} 19 \mathrm{H} 36 \mathrm{O} 2$ & 86.84 \\
\hline & $1000340-22-6^{a}$ & 9814973 & Benzoic acid, tridecyl ester & 304.24 & $\mathrm{C} 2 \mathrm{OH} 32 \mathrm{O} 2$ & 76.91 \\
\hline & $1000340-22-7^{a}$ & 64671 & Benzoic acid, tetradecyl ester & 318.26 & $\mathrm{C} 21 \mathrm{H} 34 \mathrm{O} 2$ & 81.9 \\
\hline & $1000352-68-4^{a}$ & 88368751 & Oleyl alcohol, trifluoroacetate & 364.26 & $\mathrm{C} 20 \mathrm{H} 35 \mathrm{~F} 3 \mathrm{O} 2$ & 79.42 \\
\hline & $1000356-41-5^{a}$ & 91724604 & Isophthalic acid, butyl 10-chlorodecyl ester & 396.21 & $\mathrm{C} 22 \mathrm{H} 33 \mathrm{ClO} 4$ & 71.83 \\
\hline & $1000367-89-7^{a}$ & 90471467 & Benzoic acid, dec-2-yl ester & 262.19 & $\mathrm{C} 17 \mathrm{H} 26 \mathrm{O} 2$ & 71.93 \\
\hline & $1000368-75-3^{a}$ & 91711800 & Benzoic acid, 10-chlorodecyl ester & 296.15 & $\mathrm{C} 17 \mathrm{H} 25 \mathrm{ClO} 2$ & 72.25 \\
\hline & $1000371-47-7^{a}$ & 21262075 & 1-Nonylcycloheptane & 224.25 & $\mathrm{C} 16 \mathrm{H} 32$ & 74.28 \\
\hline & $1000377-71-8^{a}$ & 91719631 & Phthalic acid, nonyl oct-3-yl ester & 404.29 & $\mathrm{C} 25 \mathrm{H} 40 \mathrm{O} 4$ & 82.23 \\
\hline & $1000406-16-5^{a}$ & 91692432 & Undec-10-ynoic acid, dodecyl ester & 350.32 & $\mathrm{C} 23 \mathrm{H} 42 \mathrm{O} 2$ & 71.23 \\
\hline & $10417-94-4$ & 446284 & cis-5,8,11,14,17-Eicosapentaenoic acid & 302.23 & $\mathrm{C} 20 \mathrm{H} 30 \mathrm{O} 2$ & 73.46 \\
\hline & $105794-58-9$ & 537071 & 1-Heptatriacotanol & 536.59 & $\mathrm{C} 37 \mathrm{H} 76 \mathrm{O}$ & 78.32 \\
\hline & $108511-83-7$ & 569871 & 2-Benzoyloxy-1,1,10-trimethyl-6,9-epidioxydecalin & 330.18 & $\mathrm{C} 20 \mathrm{H} 26 \mathrm{O} 4$ & 71.18 \\
\hline & $1129-41-5$ & 14322 & Carbamic acid, methyl-, 3-methylphenyl ester & 165.08 & $\mathrm{C} 9 \mathrm{H} 11 \mathrm{NO} 2$ & 80.66 \\
\hline & $115-89-9$ & 8291 & Diphenyl methyl phosphate & 264.06 & $\mathrm{C} 13 \mathrm{H} 13 \mathrm{O} 4 \mathrm{P}$ & 78.24 \\
\hline & $119-61-9$ & 3102 & Benzophenone & 182.07 & $\mathrm{C} 13 \mathrm{H} 10 \mathrm{O}$ & 80.07 \\
\hline & $120-46-7$ & 8433 & Dibenzoylmethane & 224.08 & $\mathrm{C} 15 \mathrm{H} 12 \mathrm{O} 2$ & 73.54 \\
\hline & $128-37-0$ & 31404 & Butylated Hydroxytoluene & 220.18 & $\mathrm{C} 15 \mathrm{H} 24 \mathrm{O}$ & 82.89 \\
\hline & 139776-09-3 & 569530 & S-Benzoyl-N-(p-nitrobenzylidene)thiohydroxylamine & 286.04 & $\mathrm{C} 14 \mathrm{H} 10 \mathrm{~N} 2 \mathrm{O} 3 \mathrm{~S}$ & 74.31 \\
\hline & $143-07-7$ & 3893 & Dodecanoic acid & 200.18 & $\mathrm{C} 12 \mathrm{H} 24 \mathrm{O} 2$ & 78.34 \\
\hline & $149180-87-0$ & 624073 & Butylaldehyde, 4-benzyloxy-4-[2,2,-dimethyl-4-dioxolanyl]- & 278.15 & $\mathrm{C} 16 \mathrm{H} 22 \mathrm{O} 4$ & 72.53 \\
\hline & $150-86-7$ & 5280435 & Phytol & 296.31 & $\mathrm{C} 20 \mathrm{H} 40 \mathrm{O}$ & 78.92 \\
\hline & $177746-99-5$ & 91692548 & Methyl 15-hydroxy-9,12-octadecadienoate & 310.25 & $\mathrm{C} 19 \mathrm{H} 34 \mathrm{O} 3$ & 76.43 \\
\hline & $20548-62-3$ & 590836 & Phthalic acid, bis(7-methyloctyl) ester & 418.31 & $\mathrm{C} 26 \mathrm{H} 42 \mathrm{O} 4$ & 78.82 \\
\hline & $22599-96-8$ & 22213932 & Cholestan-3-ol, 2-methylene-, (3.beta.,5.alpha.)- & 400.37 & $\mathrm{C} 28 \mathrm{H} 48 \mathrm{O}$ & 73.41 \\
\hline & $24560-98-3$ & 119250 & Oxiraneoctanoic acid, 3-octyl-, cis- & 298.25 & $\mathrm{C} 18 \mathrm{H} 34 \mathrm{O} 3$ & 77.99 \\
\hline
\end{tabular}




\begin{tabular}{|c|c|c|c|c|c|c|}
\hline \multirow[t]{24}{*}{ Sample } & CAS & $\begin{array}{l}\text { PubChem } \\
\text { CID }\end{array}$ & Name according to NIST & Mass & Formula & Score \\
\hline & $25360-09-2$ & 19107815 & tert-Hexadecanethiol & 258.24 & $\mathrm{C} 16 \mathrm{H} 34 \mathrm{~S}$ & 72.32 \\
\hline & $2676-41-7$ & 146287 & 6,9,12-Octadecatrienoic acid, methyl ester & 292.24 & $\mathrm{C} 19 \mathrm{H} 32 \mathrm{O} 2$ & 74.8 \\
\hline & 26896-20-8 & 62838 & Neodecanoic acid & 172.15 & $\mathrm{C} 10 \mathrm{H} 20 \mathrm{O} 2$ & 72.5 \\
\hline & $28108-99-8$ & 34148 & Phosphoric acid, (1-methylethyl)phenyl diphenyl ester & 368.12 & $\mathrm{C} 21 \mathrm{H} 21 \mathrm{O} 4 \mathrm{P}$ & 78.89 \\
\hline & 29761-21-5 & 34697 & Phosphoric acid, isodecyl diphenyl ester & 390.20 & $\mathrm{C} 22 \mathrm{H} 31 \mathrm{O} 4 \mathrm{P}$ & 73.13 \\
\hline & $334-68-9$ & 9548 & Dodecane, 1-fluoro- & 188.19 & $\mathrm{C} 12 \mathrm{H} 25 \mathrm{~F}$ & 74.35 \\
\hline & $33795-18-5$ & 214694 & Phosphonic acid, (p-hydroxyphenyl)- & 174.01 & $\mathrm{C} 6 \mathrm{H} 7 \mathrm{O} 4 \mathrm{P}$ & 76.11 \\
\hline & $3443-82-1$ & 5365676 & 9,12-Octadecadienoic acid (Z,Z)-, 2-hydroxy-1-(hydroxymethyl)ethyl ester & 354.28 & $\mathrm{C} 21 \mathrm{H} 38 \mathrm{O} 4$ & 79.87 \\
\hline & $34909-69-8$ & 631942 & Phosphoric acid, bis(4-methylphenyl) phenyl ester & 354.10 & $\mathrm{C} 20 \mathrm{H} 19 \mathrm{O} 4 \mathrm{P}$ & 83.97 \\
\hline & $373-49-9$ & 445638 & Palmitoleic acid & 254.24 & $\mathrm{C} 16 \mathrm{H} 30 \mathrm{O} 2$ & 77.31 \\
\hline & $56051-53-7$ & 554084 & $\begin{array}{l}\text { Cyclopropanebutanoic acid, 2-[[2-[[2-[(2- } \\
\text { pentylcyclopropyl)methyl]cyclopropyl]methyl]cyclopropyl]methyl]-, methyl ester }\end{array}$ & 374.32 & $\mathrm{C} 25 \mathrm{H} 42 \mathrm{O} 2$ & 75.58 \\
\hline & $57-10-3$ & 985 & n-Hexadecanoic acid & 256.24 & $\mathrm{C} 16 \mathrm{H} 32 \mathrm{O} 2$ & 74.41 \\
\hline & $57156-91-9$ & 42151 & 2,5-Octadecadiynoic acid, methyl ester & 290.23 & $\mathrm{C} 19 \mathrm{H} 30 \mathrm{O} 2$ & 71.56 \\
\hline & $60-33-3$ & 5280450 & 9,12-Octadecadienoic acid (Z,Z)- & 280.24 & $\mathrm{C} 18 \mathrm{H} 32 \mathrm{O} 2$ & 74.4 \\
\hline & $60609-53-2$ & 5364688 & 8-Hexadecenal, 14-methyl-, (Z)- & 252.25 & $\mathrm{C} 17 \mathrm{H} 32 \mathrm{O}$ & 77.49 \\
\hline & $74685-30-6$ & 5364600 & 5-Eicosene, $(\mathrm{E})$ - & 280.32 & $\mathrm{C} 20 \mathrm{H} 40$ & 80.86 \\
\hline & $76841-70-8$ & 71403428 & E-2-Hexenyl benzoate & 204.12 & $\mathrm{C} 13 \mathrm{H} 16 \mathrm{O} 2$ & 73.99 \\
\hline & $77-90-7$ & 6505 & Tributyl acetylcitrate & 402.23 & C20H34O8 & 71.72 \\
\hline & $78-31-9$ & 6528 & Phosphoric acid, 4-methylphenyl diphenyl ester & 340.09 & C19H17O4P & 79.33 \\
\hline & $816-19-3$ & 102491 & Hexanoic acid, 2-ethyl-, methyl ester & 158.13 & C9H18O2 & 71.83 \\
\hline & $82304-66-3$ & 545303 & 7,9-Di-tert-butyl-1-oxaspiro(4,5)deca-6,9-diene-2,8-dione & 276.17 & $\mathrm{C} 17 \mathrm{H} 24 \mathrm{O} 3$ & 71.69 \\
\hline & $84-77-5$ & 6788 & Didecyl phthalate & 446.34 & $\mathrm{C} 28 \mathrm{H} 46 \mathrm{O} 4$ & 83.9 \\
\hline & $85763-57-1$ & 33865 & 11-Methyldodecanol & 200.21 & $\mathrm{C} 13 \mathrm{H} 28 \mathrm{O}$ & 78.32 \\
\hline \multirow[t]{13}{*}{ PVC 4} & $1225365^{a}$ & 520263 & Phosphoric acid, 2-methylphenyl diphenyl ester & 340.09 & $\mathrm{C} 19 \mathrm{H} 17 \mathrm{O} 4 \mathrm{P}$ & 86.56 \\
\hline & $1919690^{a}$ & 81591 & Benzoic acid, heptyl ester & 220.15 & $\mathrm{C} 14 \mathrm{H} 20 \mathrm{O} 2$ & 84.53 \\
\hline & $1000308-89-8^{a}$ & 3024584 & Phthalic acid, decyl nonyl ester & 432.32 & $\mathrm{C} 27 \mathrm{H} 44 \mathrm{O} 4$ & 70.78 \\
\hline & $1000339-40-5^{a}$ & 12151622 & 1,2-Cyclohexanedicarboxylic acid, dinonyl ester & 424.36 & $\mathrm{C} 26 \mathrm{H} 48 \mathrm{O} 4$ & 74.42 \\
\hline & $1000339-74-3^{a}$ & 91721826 & 1,2-Cyclohexanedicarboxylic acid, cyclohexylmethyl nonyl ester & 394.31 & $\mathrm{C} 24 \mathrm{H} 42 \mathrm{O} 4$ & 77.87 \\
\hline & $1000340-22-6^{a}$ & 9814973 & Benzoic acid, tridecyl ester & 304.24 & $\mathrm{C} 20 \mathrm{H} 32 \mathrm{O} 2$ & 80.46 \\
\hline & $1000353-65-9^{a}$ & 91714177 & Adipic acid, 3-heptyl tetradecyl ester & 440.39 & $\mathrm{C} 27 \mathrm{H} 52 \mathrm{O} 4$ & 76.38 \\
\hline & $1000367-91-3^{a}$ & 103653 & Benzoic acid, 2-methylbutyl ester & 192.12 & $\mathrm{C} 12 \mathrm{H} 16 \mathrm{O} 2$ & 77.5 \\
\hline & $1000368-69-4^{a}$ & 243678 & Benzoic acid, hept-2-yl ester & 220.15 & $\mathrm{C} 14 \mathrm{H} 20 \mathrm{O} 2$ & 76.04 \\
\hline & $1000371-07-7^{a}$ & 91719575 & Phthalic acid, 5-methylhex-2-yl heptadecyl ester & 502.40 & $\mathrm{C} 32 \mathrm{H} 54 \mathrm{O} 4$ & 75.96 \\
\hline & $18699-48-4$ & 29218 & 1,4-Benzenedicarboxylic acid, bis(2-methylpropyl) ester & 278.15 & $\mathrm{C} 16 \mathrm{H} 22 \mathrm{O} 4$ & 90.68 \\
\hline & $20548-62-3$ & 590836 & Phthalic acid, bis(7-methyloctyl) ester & 418.31 & $\mathrm{C} 26 \mathrm{H} 42 \mathrm{O} 4$ & 81.45 \\
\hline & $29761-21-5$ & 34697 & Phosphoric acid, isodecyl diphenyl ester & 390.20 & $\mathrm{C} 22 \mathrm{H} 31 \mathrm{O} 4 \mathrm{P}$ & 80.26 \\
\hline
\end{tabular}




\begin{tabular}{|c|c|c|c|c|c|c|}
\hline Sample & CAS & $\begin{array}{l}\text { PubChem } \\
\text { CID }\end{array}$ & Name according to NIST & Mass & Formula & Score \\
\hline & $34909-69-8$ & 631942 & Phosphoric acid, bis(4-methylphenyl) phenyl ester & 354.10 & $\mathrm{C} 20 \mathrm{H} 19 \mathrm{O} 4 \mathrm{P}$ & 88.72 \\
\hline & $59736-57-1$ & 570433 & Benzoic acid 2-methylpentyl ester & 206.13 & $\mathrm{C} 13 \mathrm{H} 18 \mathrm{O} 2$ & 83.13 \\
\hline & $76841-70-8$ & 71403428 & E-2-Hexenyl benzoate & 204.12 & $\mathrm{C} 13 \mathrm{H} 16 \mathrm{O} 2$ & 73.45 \\
\hline & $77-90-7$ & 6505 & Tributyl acetylcitrate & 402.23 & $\mathrm{C} 20 \mathrm{H} 34 \mathrm{O} 8$ & 83.62 \\
\hline & $78-31-9$ & 6528 & Phosphoric acid, 4-methylphenyl diphenyl ester & 340.09 & $\mathrm{C} 19 \mathrm{H} 17 \mathrm{O} 4 \mathrm{P}$ & 83.33 \\
\hline & $84-64-0$ & 6779 & 1,2-Benzenedicarboxylic acid, butyl cyclohexyl ester & 304.17 & $\mathrm{C} 18 \mathrm{H} 24 \mathrm{O} 4$ & 75.60 \\
\hline & 84-76-4 & 6787 & 1,2-Benzenedicarboxylic acid, dinonyl ester & 418.31 & $\mathrm{C} 26 \mathrm{H} 42 \mathrm{O} 4$ & 83.79 \\
\hline & $85-68-7$ & 2347 & Benzyl butyl phthalate & 312.14 & $\mathrm{C} 19 \mathrm{H} 20 \mathrm{O} 4$ & 71.83 \\
\hline & $94-50-8$ & 66751 & Benzoic acid, octyl ester & 234.16 & $\mathrm{C} 15 \mathrm{H} 22 \mathrm{O} 2$ & 85.23 \\
\hline \multirow[t]{2}{*}{ PUR 1} & $112-61-8$ & 8201 & Methyl stearate & 298.29 & $\mathrm{C} 19 \mathrm{H} 38 \mathrm{O} 2$ & 75.92 \\
\hline & $128-37-0$ & 31404 & Butylated Hydroxytoluene & 220.18 & $\mathrm{C} 15 \mathrm{H} 24 \mathrm{O}$ & 89.33 \\
\hline \multirow[t]{13}{*}{ PUR 2} & $1000128-20-5^{a}$ & none & $(+,-)$-Epi-perhydrohistrionicotoxin & 295.29 & $\mathrm{C} 19 \mathrm{H} 37 \mathrm{NO}$ & 71.38 \\
\hline & $1000195-87-0^{a}$ & 550132 & $\begin{array}{l}\text { 8,14-Seco-3,19-epoxyandrostane-8,14-dione, } 17 \text {-acetoxy-3.beta.-methoxy-4,4- } \\
\text { dimethyl- }\end{array}$ & 420.25 & $\mathrm{C} 24 \mathrm{H} 36 \mathrm{O} 6$ & 70.01 \\
\hline & $1000303-02-6^{a}$ & 6423312 & 7-Amino-1,3-dihydro-indol-2-one & 148.06 & $\mathrm{C} 8 \mathrm{H} 8 \mathrm{~N} 2 \mathrm{O}$ & 75.44 \\
\hline & $128-37-0$ & 31404 & Butylated Hydroxytoluene & 220.18 & $\mathrm{C} 15 \mathrm{H} 24 \mathrm{O}$ & 92.86 \\
\hline & $149-57-5$ & 8697 & Hexanoic acid, 2-ethyl- & 144.12 & $\mathrm{C} 8 \mathrm{H} 16 \mathrm{O} 2$ & 71.15 \\
\hline & $2456-81-7$ & 75567 & Pyridine, 4-(1-pyrrolidinyl)- & 148.10 & $\mathrm{C} 9 \mathrm{H} 12 \mathrm{~N} 2$ & 81.91 \\
\hline & $2566-91-8$ & 6451414 & Oxiraneoctanoic acid, 3-octyl-, methyl ester, cis- & 312.27 & $\mathrm{C} 19 \mathrm{H} 36 \mathrm{O} 3$ & 76.43 \\
\hline & $301-02-0$ & 5283387 & 9-Octadecenamide, $(\mathrm{Z})$ - & 281.27 & $\mathrm{C} 18 \mathrm{H} 35 \mathrm{NO}$ & 80.39 \\
\hline & $5129-61-3$ & 110444 & Heptadecanoic acid, 16-methyl-, methyl ester & 298.29 & $\mathrm{C} 19 \mathrm{H} 38 \mathrm{O} 2$ & 72.06 \\
\hline & $584-84-9$ & 11443 & Benzene, 2,4-diisocyanato-1-methyl- & 174.04 & $\mathrm{C} 9 \mathrm{H} 6 \mathrm{~N} 2 \mathrm{O} 2$ & 85.39 \\
\hline & $61338-98-5$ & 547892 & Benzeneethanamine, 2-fluoro-.beta.,3,4-trihydroxy-N-isopropyl- & 229.11 & C11H16FNO3 & 74.85 \\
\hline & $76-25-5$ & 6436 & Triamcinolone Acetonide & 434.21 & $\mathrm{C} 24 \mathrm{H} 31 \mathrm{FO} 6$ & 70.57 \\
\hline & $823-40-5$ & 13205 & 1,3-Benzenediamine, 2-methyl- & 122.08 & $\mathrm{C} 7 \mathrm{H} 10 \mathrm{~N} 2$ & 71.37 \\
\hline \multirow[t]{6}{*}{ PUR 3} & $1000370-31-1^{a}$ & 458684 & 4,4'-Di-tert-butyl-diphenylamine & 281.21 & $\mathrm{C} 20 \mathrm{H} 27 \mathrm{~N}$ & 70.45 \\
\hline & $1000370-31-3^{a}$ & 117942 & Tert-octyldiphenylamine & 281.21 & $\mathrm{C} 20 \mathrm{H} 27 \mathrm{~N}$ & 79.04 \\
\hline & $1000400-90-8^{a}$ & 291360 & benzaldehyde, 4-(ethylphenylamino)- & 225.12 & $\mathrm{C} 15 \mathrm{H} 15 \mathrm{NO}$ & 75.37 \\
\hline & $1000408-33-2^{a}$ & 91739821 & 2-[2-Methoxy-5-(1,1,3,3-tetramethylbutyl)phenyl]-2H-benzotriazole & 337.22 & $\mathrm{C} 21 \mathrm{H} 27 \mathrm{~N} 3 \mathrm{O}$ & 77.39 \\
\hline & $6386-38-5$ & 62603 & Benzenepropanoic acid, 3,5-bis(1,1-dimethylethyl)-4-hydroxy-, methyl ester & 292.20 & $\mathrm{C} 18 \mathrm{H} 28 \mathrm{O} 3$ & 70.30 \\
\hline & $78-40-0$ & 6535 & Triethyl phosphate & 182.07 & $\mathrm{C} 6 \mathrm{H} 15 \mathrm{O} 4 \mathrm{P}$ & 74.93 \\
\hline \multirow[t]{2}{*}{ PUR 4} & $128-37-0$ & 31404 & Butylated Hydroxytoluene & 220.18 & $\mathrm{C} 15 \mathrm{H} 24 \mathrm{O}$ & 77.47 \\
\hline & $729-43-1$ & 5484329 & Ethanone, 1-phenyl-, (1-phenylethylidene)hydrazone & 236.13 & $\mathrm{C} 16 \mathrm{H} 16 \mathrm{~N} 2$ & 81.99 \\
\hline \multirow[t]{2}{*}{ PLA 1} & $1000382-54-3^{a}$ & 91693137 & Carbonic acid, eicosyl vinyl ester & 368.33 & $\mathrm{C} 23 \mathrm{H} 44 \mathrm{O} 3$ & 78.36 \\
\hline & $554-12-1$ & 11124 & Methyl propionate & 88.05 & $\mathrm{C} 4 \mathrm{H} 8 \mathrm{O} 2$ & 74.34 \\
\hline PLA 2 & $554-12-1$ & 11124 & Methyl propionate & 88.05 & $\mathrm{C} 4 \mathrm{H} 8 \mathrm{O} 2$ & 72.33 \\
\hline
\end{tabular}




\begin{tabular}{|c|c|c|c|c|c|c|}
\hline Sample & CAS & $\begin{array}{l}\text { PubChem } \\
\text { CID }\end{array}$ & Name according to NIST & Mass & Formula & Score \\
\hline \multirow[t]{7}{*}{ PLA 4} & $112-67-4$ & 8206 & Palmitoyl chloride & 274.21 & $\mathrm{C} 16 \mathrm{H} 31 \mathrm{ClO}$ & 70.25 \\
\hline & $112-80-1$ & 445639 & Oleic Acid & 282.26 & $\mathrm{C} 18 \mathrm{H} 34 \mathrm{O} 2$ & 74.11 \\
\hline & $143-07-7$ & 3893 & Dodecanoic acid & 200.18 & $\mathrm{C} 12 \mathrm{H} 24 \mathrm{O} 2$ & 74.32 \\
\hline & $1673-08-1$ & 74288 & Hexadecanoic acid, cyclohexyl ester & 338.32 & $\mathrm{C} 22 \mathrm{H} 42 \mathrm{O} 2$ & 70.45 \\
\hline & $5129-61-3$ & 110444 & Heptadecanoic acid, 16-methyl-, methyl ester & 298.29 & $\mathrm{C} 19 \mathrm{H} 38 \mathrm{O} 2$ & 82.05 \\
\hline & $57-10-3$ & 985 & n-Hexadecanoic acid & 256.24 & $\mathrm{C} 16 \mathrm{H} 32 \mathrm{O} 2$ & 84.18 \\
\hline & $57-11-4$ & 5281 & Octadecanoic acid & 284.27 & $\mathrm{C} 18 \mathrm{H} 36 \mathrm{O} 2$ & 71.32 \\
\hline
\end{tabular}

381 
382 Table S4. Cell viability (\%) of the cytotoxic samples in the reporter gene assay experiments. PPAR $\gamma=$ peroxisome proliferator receptor 383 gamma, $\mathrm{GR}=$ glucocorticoid receptor, $\mathrm{SD}=$ standard deviation.

\begin{tabular}{|c|c|c|c|c|c|c|c|c|c|c|c|c|c|c|c|c|c|c|}
\hline $\begin{array}{l}\text { PPAR } \gamma \text { CALUX } \\
\text { mg plastic well }{ }^{-1}\end{array}$ & PS 2 & & & PP 3 & & & PP 4 & & & PVC 2 & & & PLA1 & & & PVC 4 & & \\
\hline & mean & SD & $\mathrm{n}$ & mean & SD & $\mathrm{n}$ & mean & SD & $\mathrm{n}$ & mean & SD & $\mathrm{n}$ & mean & SD & $\mathrm{n}$ & mean & SD & $\mathrm{n}$ \\
\hline 1.5 & 9.7 & 5 & 16 & 13.9 & 7.6 & 12 & 22.6 & 13.2 & 16 & 44.9 & 21.6 & 20 & 25.7 & 7.9 & 12 & 107.9 & 14.9 & 20 \\
\hline 0.75 & 68.4 & 14.2 & 16 & 41 & 21 & 12 & 14.9 & 3.8 & 16 & 106.8 & 15.4 & 20 & 103.2 & 15.1 & 12 & 120.9 & 20.3 & 20 \\
\hline 0.38 & 108.2 & 10.6 & 16 & 86.9 & 20.8 & 12 & 19.9 & 9.8 & 16 & 117.5 & 16.3 & 20 & 111.6 & 15.5 & 12 & 126.7 & 20.4 & 20 \\
\hline 0.19 & 120.5 & 7.1 & 16 & 106 & 16.6 & 12 & 97.7 & 25.4 & 16 & 123.9 & 19.9 & 20 & 118.4 & 21 & 12 & 126.6 & 30.5 & 20 \\
\hline 0.09 & 121.7 & 6.3 & 16 & 123 & 20.4 & 12 & 109.8 & 11.8 & 16 & 125.5 & 25.1 & 20 & 128.6 & 24.5 & 12 & 121.8 & 34.6 & 20 \\
\hline 0.04 & 116.8 & 15.7 & 16 & 104 & 26.4 & 12 & 101.8 & 10.9 & 16 & 115.4 & 34 & 20 & 131.9 & 21.5 & 12 & 101.8 & 27.2 & 20 \\
\hline \multirow[t]{2}{*}{$\begin{array}{l}\text { GR CALUX } \\
\text { mg plastic well }\end{array}$} & PS 2 & & & PP 3 & & & PP 4 & & & PVC 2 & & & PLA 1 & & & PVC 4 & & \\
\hline & mean & SD & $\mathrm{n}$ & mean & SD & $\mathrm{n}$ & mean & SD & $\mathrm{n}$ & mean & SD & $\mathrm{n}$ & mean & SD & $\mathrm{n}$ & mean & SD & $\mathrm{n}$ \\
\hline 1.5 & 7 & 7.1 & 12 & 43.2 & 13 & 12 & 8.8 & 8.6 & 16 & 52 & 38.7 & 16 & 45.1 & 42.1 & 12 & 71 & 20 & 12 \\
\hline 0.75 & 76.1 & 45.9 & 12 & 57.3 & 13.4 & 12 & 13 & 9.1 & 16 & 109.8 & 10.6 & 16 & 104.3 & 9.3 & 12 & 96 & 9 & 12 \\
\hline 0.38 & 101 & 38.4 & 12 & 88.9 & 15.3 & 12 & 17.5 & 25.2 & 16 & 118.3 & 7.6 & 16 & 112.7 & 6.9 & 12 & 110 & 10 & 12 \\
\hline 0.19 & 117 & 23.7 & 12 & 110.4 & 5.8 & 12 & 96.8 & 18.4 & 16 & 117.2 & 8.5 & 16 & 112.2 & 6 & 12 & 116 & 6 & 12 \\
\hline 0.09 & 113.3 & 19.6 & 12 & 115.2 & 7.5 & 12 & 118.4 & 17.8 & 16 & 118.9 & 8 & 16 & 113.9 & 6.9 & 12 & 113 & 12 & 12 \\
\hline 0.04 & 111.2 & 31.7 & 12 & 103.8 & 10.4 & 12 & 105.8 & 13.4 & 16 & 104.5 & 6.8 & 16 & 107.5 & 9.6 & 12 & 102 & 11 & 12 \\
\hline
\end{tabular}


Table S5. Comparison of the adipogenic effects of plastic extracts and the abundance of three metabolic disrupting chemicals detected in 386 at least three samples, diphenyl phosphate (DPP), 2-ethylhexyl diphenyl phosphate (EHDP), and triphenyl phosphate (TPP) in the LC-QTOFMS/MS.

\begin{tabular}{|c|c|c|c|c|c|c|c|c|c|c|c|c|c|c|c|c|}
\hline \multirow[b]{2}{*}{ Samples } & \multicolumn{2}{|c|}{ Lipid droplet count } & \multicolumn{2}{|c|}{ Raw abundance } & \multirow[b]{2}{*}{$\operatorname{DPP} 3^{a}$} & \multirow[b]{2}{*}{$\mathrm{DPP} 4^{a}$} & \multirow[b]{2}{*}{ EHDP } & \multirow[b]{2}{*}{$\operatorname{TPP} 1^{a}$} & \multirow[b]{2}{*}{$\operatorname{TPP} 2^{a}$} & \multirow[b]{2}{*}{$\operatorname{TPP} 3^{a}$} & \multirow[b]{2}{*}{$\operatorname{TPP} 4^{a}$} & \multirow[b]{2}{*}{$\operatorname{TPP} 5^{a}$} & \multirow[b]{2}{*}{$\operatorname{TPP} 6^{a}$} & \multirow[b]{2}{*}{$\operatorname{TPP} 7^{a}$} & \multirow[b]{2}{*}{$\operatorname{TPP} 8^{a}$} & \multirow[b]{2}{*}{$\operatorname{TPP} 9^{a}$} \\
\hline & Median & $\mathrm{EC}_{50}$ & $\mathrm{DPP} 1^{a}$ & $\mathrm{DPP} 2^{a}$ & & & & & & & & & & & & \\
\hline$\overline{\text { HDPE } 1}$ & 164.6 & 3.1 & 0 & 0 & $<\mathrm{LOD}$ & $<\mathrm{LOD}$ & 0 & 0 & 0 & 1542 & 0 & 0 & 0 & 0 & 0 & 0 \\
\hline HDPE 2 & 161.4 & 3.1 & 0 & 0 & $<\mathrm{LOD}$ & $<\mathrm{LOD}$ & 0 & 0 & 0 & 0 & $<\mathrm{LOD}$ & 0 & 0 & 0 & 0 & 0 \\
\hline HDPE 3 & 360.8 & 3.1 & 0 & 0 & $<\mathrm{LOD}$ & $<\mathrm{LOD}$ & 0 & 0 & 0 & 0 & 0 & 0 & 0 & 2426 & 0 & 0 \\
\hline HDPE 4 & 369.1 & 3.1 & 0 & 0 & $<$ LOD & $<\mathrm{LOD}$ & 4.7 & 0 & 0 & 0 & $<\mathrm{LOD}$ & 0 & 0 & 0 & 0 & 0 \\
\hline LDPE 1 & 447.3 & 3.1 & 0 & 0 & $<\mathrm{LOD}$ & $<\mathrm{LOD}$ & 5.7 & 0 & 0 & 0 & $<\mathrm{LOD}$ & 0 & 0 & 0 & 0 & 0 \\
\hline LDPE 2 & 373.2 & 3.1 & 0 & 0 & $<\mathrm{LOD}$ & $<\mathrm{LOD}$ & 0 & 0 & 0 & 24.6 & 0 & 0 & 0 & 0 & 0 & 137.4 \\
\hline LDPE 3 & 294.4 & 3.1 & 0 & 0 & $<\mathrm{LOD}$ & $<\mathrm{LOD}$ & 0 & 0 & 0 & 0 & 0 & 0 & 0 & $<\mathrm{LOD}$ & 0 & 0 \\
\hline LDPE 4 & 1352 & 1.59 & 0 & 0 & $<\mathrm{LOD}$ & $<\mathrm{LOD}$ & 0 & 0 & 0 & 0 & 974.2 & 0 & 0.1 & 0 & 0 & 0 \\
\hline PS 1 & 419.9 & 3.1 & 0 & 0 & $<\mathrm{LOD}$ & 0 & 0 & 14.6 & 0 & 0 & 0 & 0 & 0 & 0 & 0 & 0 \\
\hline PS 2 & 1333 & 2.05 & 9.8 & 0 & $<\mathrm{LOD}$ & $<\mathrm{LOD}$ & 0 & 0 & 16.7 & 0 & 0 & 0 & 0 & 0 & $<\mathrm{LOD}$ & 0 \\
\hline PS 3 & 186.1 & 3.1 & 0 & 0 & $<\mathrm{LOD}$ & 0 & 0 & 0 & 0 & 0 & 0 & 0 & 0 & 0 & 0 & 0 \\
\hline PS 4 & 123.7 & 3.1 & 0 & 0 & 0 & $<\mathrm{LOD}$ & 0 & 0 & 0 & 0 & $<\mathrm{LOD}$ & 0 & 0 & 0 & 0 & 0 \\
\hline PP 1 & 197.3 & 3.1 & 0 & 0 & $<\mathrm{LOD}$ & $<$ LOD & 0 & 0 & 1633 & 0 & $<$ LOD & 0 & 0 & 0 & 0 & 984.3 \\
\hline PP 2 & 413 & 3.1 & 0 & 0 & 0 & $<\mathrm{LOD}$ & 0 & 0 & 0 & 2.2 & $<\mathrm{LOD}$ & 0 & 0 & 0 & 0 & 0 \\
\hline PP 3 & 1742 & 1.4 & 0 & 0 & $<\mathrm{LOD}$ & 0 & 0 & 1184 & 0 & 59.3 & $<\mathrm{LOD}$ & 0 & 0 & 0 & 0 & 0 \\
\hline PP 4 & 2927 & 0.4 & 0 & 0 & 0 & $<\mathrm{LOD}$ & 0 & 0 & 0 & 0 & $<\mathrm{LOD}$ & 0 & 0 & 0 & 0 & 0 \\
\hline PP 5 & 119.1 & 3.1 & 0 & 0 & 0 & $<\mathrm{LOD}$ & 0 & 0 & 0 & 0 & $<\mathrm{LOD}$ & 0 & 0 & 0 & 0 & 0 \\
\hline PET 1 & 99.12 & 3.1 & 0 & 0 & 0 & $<\mathrm{LOD}$ & 0 & 0 & 0 & 0 & $<\mathrm{LOD}$ & 0 & 0 & 0 & 0 & 0 \\
\hline PET 2 & 185 & 3.1 & 0 & 0 & 0 & $<\mathrm{LOD}$ & 0 & 0 & 0 & 0 & $<\mathrm{LOD}$ & 0 & 0 & 0 & 0 & 0 \\
\hline PET 3 & 139.4 & 3.1 & 0 & 0 & 0 & $<\mathrm{LOD}$ & 0 & 0 & 0 & 0 & $<\mathrm{LOD}$ & 0 & 0 & 0 & 0 & 0 \\
\hline PET 4 & 122.4 & 3.1 & 0 & 0 & $<\mathrm{LOD}$ & $<\mathrm{LOD}$ & 0 & 0 & 0 & 0 & 0 & 0 & 0 & 0 & 0 & 0 \\
\hline PET 5 & 183.7 & 3.1 & 0 & 0 & $<\mathrm{LOD}$ & $<\mathrm{LOD}$ & 0 & 0 & 0 & 0 & $<\mathrm{LOD}$ & 0 & 0 & $<\mathrm{LOD}$ & 0 & 0 \\
\hline
\end{tabular}




\begin{tabular}{|c|c|c|c|c|c|c|c|c|c|c|c|c|c|c|c|c|}
\hline \multirow[b]{2}{*}{ Samples } & \multicolumn{2}{|c|}{ Lipid droplet count } & \multicolumn{2}{|c|}{ Raw abundance } & \multirow[b]{2}{*}{$\mathrm{DPP} 3^{a}$} & \multirow[b]{2}{*}{$\operatorname{DPP} 4^{a}$} & \multirow[b]{2}{*}{ EHDP } & \multirow[b]{2}{*}{$\operatorname{TPP} 1^{a}$} & \multirow[b]{2}{*}{ TPP $2^{a}$} & \multirow[b]{2}{*}{ ТРP $3^{a}$} & \multirow[b]{2}{*}{ ТPP $4^{a}$} & \multirow[b]{2}{*}{ TPP $5^{a}$} & \multirow[b]{2}{*}{ TPP $6^{a}$} & \multirow[b]{2}{*}{$\operatorname{TPP} 7^{a}$} & \multirow[b]{2}{*}{ ТPР $8^{a}$} & \multirow[b]{2}{*}{ ТPP $9^{a}$} \\
\hline & Median & $\mathrm{EC}_{50}$ & $\mathrm{DPP} 1^{a}$ & $\operatorname{DPP} 2^{a}$ & & & & & & & & & & & & \\
\hline$\overline{\mathrm{PVC}} 1$ & 171.4 & 3.1 & 0 & 0 & 0 & $<\mathrm{LOD}$ & 0 & 0 & 0 & 0 & $<\mathrm{LOD}$ & 0 & 0 & 0 & $\overline{0}$ & $\overline{0}$ \\
\hline PVC 2 & 3302 & 0.85 & 4251 & 2466 & 437,900 & $<\mathrm{LOD}$ & 0 & 0 & 0 & 356,178 & $<\mathrm{LOD}$ & 0 & 0 & 0 & 0 & 0 \\
\hline PVC 3 & 903.5 & 1 & 0 & 0 & 0 & $<\mathrm{LOD}$ & 0 & 0 & 0 & 0 & 554,290 & 0 & 27.1 & 0 & 0 & 0 \\
\hline PVC 4 & 3044 & 0.53 & 0 & 0 & $<\mathrm{LOD}$ & 315,591 & 2040 & 0 & 0 & 0 & $<\mathrm{LOD}$ & 4094 & 544,411 & $<\mathrm{LOD}$ & 4257 & 10.7 \\
\hline PUR 1 & 1340 & 0.61 & 0 & 0 & 0 & $<\mathrm{LOD}$ & 1.6 & 0 & 0 & 63.9 & $<\mathrm{LOD}$ & 771.1 & 0 & 0 & 0 & 0 \\
\hline PUR 2 & 969.4 & 0.33 & 0 & 0 & 0 & $<$ LOD & $<\mathrm{LOD}$ & 0 & 0 & 0 & $<\mathrm{LOD}$ & 22.3 & 0 & 0 & 0 & 0 \\
\hline PUR 3 & 1186 & 0.32 & 0 & 0 & $<\mathrm{LOD}$ & $<\mathrm{LOD}$ & 65.8 & 95.7 & 1.4 & 31.3 & 0 & 0 & 0 & 0 & 0 & 0 \\
\hline PUR 4 & 2195 & 1.05 & 0 & 0 & 0 & $<\mathrm{LOD}$ & 438.7 & 0 & 0 & 0 & $<\mathrm{LOD}$ & 0 & 0.1 & 0 & 0 & 0 \\
\hline PLA 1 & 281.7 & 3.1 & 0 & 0 & $<\mathrm{LOD}$ & $<\mathrm{LOD}$ & 0 & 0 & 0 & 0 & 0 & 0 & 33.4 & 0 & $<\mathrm{LOD}$ & 0 \\
\hline PLA 2 & 203.7 & 3.1 & 0 & 0 & $<\mathrm{LOD}$ & $<$ LOD & 0 & 0 & 0 & 0 & 0 & 0 & 0 & $<\mathrm{LOD}$ & 0 & 0 \\
\hline PLA 3 & \multicolumn{16}{|c|}{ not analyzed via LC-QTOF-MS/MS } \\
\hline PLA 4 & 271.8 & 3.1 & 0 & 0 & $<\mathrm{LOD}$ & $<\mathrm{LOD}$ & 0 & 0 & 0 & 0 & $<\mathrm{LOD}$ & 0 & 0 & 0 & 0 & 0 \\
\hline
\end{tabular}




\section{References}

390 1. Zebisch, K.; Voigt, V.; Wabitsch, M.; Brandsch, M., Protocol for effective

391 differentiation of 3T3-L1 cells to adipocytes. Anal Biochem 2012, 425, (1), 88-90.

392 2. Chappell, V. A.; Janesick, A.; Blumberg, B.; Fenton, S. E., Tetrabromobisphenol-A 393 promotes early adipogenesis and lipogenesis in 3T3-L1 cells. Toxicol Sci 2018, 166, (2), 332394344.

395 3. Boucher, J. G.; Boudreau, A.; Ahmed, S.; Atlas, E., In vitro effects of Bisphenol A 396 beta-D-glucuronide (BPA-G) on adipogenesis in human and murine preadipocytes. Environ 397 Health Perspect 2015, 123, (12), 1287-93.

$398 \quad$ 4. Kassotis, C. D.; Masse, L.; Kim, S.; Schlezinger, J. J.; Webster, T. F.; Stapleton, H. M., 399 Characterization of adipogenic chemicals in three different cell culture systems: Implications 400 for reproducibility based on cell source and handling. Sci Rep 2017, 7, 42104.

401 5. Sargis, R. M.; Johnson, D. N.; Choudhury, R. A.; Brady, M. J., Environmental 402 endocrine disruptors promote adipogenesis in the 3T3-L1 cell line through glucocorticoid 403 receptor activation. Obesity 2010, 18, (7), 1283-8.

$404 \quad$ 6. $\quad$ Pomatto, V.; Cottone, E.; Cocci, P.; Mozzicafreddo, M.; Mosconi, G.; Nelson, E. R.; 405 Palermo, F. A.; Bovolin, P., Plasticizers used in food-contact materials affect adipogenesis in 406 3T3-L1 cells. J Steroid Biochem Mol Biol 2018, 178, 322-332.

407 7. Riu, A.; Grimaldi, M.; le Maire, A.; Bey, G.; Phillips, K.; Boulahtouf, A.; Perdu, E.; 408 Zalko, D.; Bourguet, W.; Balaguer, P., Peroxisome proliferator-activated receptor gamma is a 409 target for halogenated analogs of bisphenol A. Environ Health Perspect 2011, 119, (9), 122741032.

$411 \quad 8 . \quad$ Kassotis, C. D.; Hoffman, K.; Volker, J.; Pu, Y.; Veiga-Lopez, A.; Kim, S. M.; 412 Schlezinger, J. J.; Bovolin, P.; Cottone, E.; Saraceni, A.; Scandiffio, R.; Atlas, E.; Leingartner, 413 K.; Krager, S.; Tischkau, S. A.; Ermler, S.; Legler, J.; Chappell, V. A.; Fenton, S. E.; Mesmar, 414 F.; Bondesson, M.; Fernandez, M. F.; Stapleton, H. M., Reproducibility of adipogenic 415 responses to metabolism disrupting chemicals in the 3T3-L1 pre-adipocyte model system: An 416 interlaboratory study. Toxicology 2021, 461, 152900.

417 9. Adomshick, V.; Pu, Y.; Veiga-Lopez, A., Automated lipid droplet quantification 418 system for phenotypic analysis of adipocytes using CellProfiler. Toxicol Mech Methods 2020, $41930,(5), 378-387$.

420 10. Zimmermann, L.; Dombrowski, A.; Volker, C.; Wagner, M., Are bioplastics and plant421 based materials safer than conventional plastics? In vitro toxicity and chemical composition. 422 Environ Int 2020, 145, 106066.

423 11. Zimmermann, L.; Bartosova, Z.; Braun, K.; Oehlmann, J.; Volker, C.; Wagner, M., 424 Plastic products leach chemicals that induce in vitro toxicity under realistic use conditions. 425 Environ Sci Technol 2021, 55, (17), 11814-11823. 
12. Schymanski, E. L.; Singer, H. P.; Slobodnik, J.; Ipolyi, I. M.; Oswald, P.; Krauss, M.; Schulze, T.; Haglund, P.; Letzel, T.; Grosse, S.; Thomaidis, N. S.; Bletsou, A.; Zwiener, C.; Ibanez, M.; Portoles, T.; de Boer, R.; Reid, M. J.; Onghena, M.; Kunkel, U.; Schulz, W.; Guillon, A.; Noyon, N.; Leroy, G.; Bados, P.; Bogialli, S.; Stipanicev, D.; Rostkowski, P.; Hollender, J., Non-target screening with high-resolution mass spectrometry: critical review using a collaborative trial on water analysis. Anal Bioanal Chem 2015, 407, (21), 6237-55.

13. Amato, A. A.; Wheeler, H. B.; Blumberg, B., Obesity and endocrine-disrupting chemicals. Endocr Connect 2021, 10, (2), R87-R105.

14. Zimmermann, L.; Dierkes, G.; Ternes, T. A.; Volker, C.; Wagner, M., Benchmarking the in vitro toxicity and chemical composition of plastic consumer products. Environ Sci Technol 2019, 53, (19), 11467-11477.

15. Kassotis, C. D.; Hoffman, K.; Stapleton, H. M., Characterization of adipogenic activity of house dust extracts and semi-volatile indoor contaminants in 3T3-L1 cells. Environ Sci Technol 2017, 51, (15), 8735-8745.

16. Sun, W.; Duan, X.; Chen, H.; Zhang, L.; Sun, H., Adipogenic activity of 2-ethylhexyl diphenyl phosphate via peroxisome proliferator-activated receptor gamma pathway. Sci Total Environ 2020, 711, 134810.

17. Blanco, J.; Guardia-Escote, L.; Mulero, M.; Basaure, P.; Biosca-Brull, J.; Cabre, M.; Colomina, M. T.; Domingo, J. L.; Sanchez, D. J., Obesogenic effects of chlorpyrifos and its metabolites during the differentiation of 3T3-L1 preadipocytes. Food Chem Toxicol 2020, 137, 111171.

18. Sun, Z.; Cao, H.; Liu, Q. S.; Liang, Y.; Fiedler, H.; Zhang, J.; Zhou, Q.; Jiang, G., 4Hexylphenol influences adipogenic differentiation and hepatic lipid accumulation in vitro. Environ Pollut 2021, 268, (Pt A), 115635.

19. Kassotis, C. D.; Kollitz, E. M.; Ferguson, P. L.; Stapleton, H. M., Nonionic ethoxylated surfactants induce adipogenesis in 3T3-L1 cells. Toxicol Sci 2018, 162, (1), 124-136.

20. Hao, C. J.; Cheng, X. J.; Xia, H. F.; Ma, X., The endocrine disruptor 4-nonylphenol promotes adipocyte differentiation and induces obesity in mice. Cell Physiol Biochem 2012, $30,(2), 382-94$.

21. Janesick, A. S.; Dimastrogiovanni, G.; Vanek, L.; Boulos, C.; Chamorro-Garcia, R.; Tang, W.; Blumberg, B., On the utility of ToxCast and ToxPi as methods for identifying new obesogens. Environ Health Perspect 2016, 124, (8), 1214-26.

22. Andrews, F. V.; Kim, S. M.; Edwards, L.; Schlezinger, J. J., Identifying adipogenic chemicals: Disparate effects in 3T3-L1, OP9 and primary mesenchymal multipotent cell models. Toxicol In Vitro 2020, 67, 104904.

23. Bastos Sales, L.; Kamstra, J. H.; Cenijn, P. H.; van Rijt, L. S.; Hamers, T.; Legler, J., Effects of endocrine disrupting chemicals on in vitro global DNA methylation and adipocyte differentiation. Toxicol In Vitro 2013, 27, (6), 1634-43. 

3T3-L1 preadipocytes: A High Content Cellomics and metabolomic analysis. Toxicol In Vitro 2016, 32, 297-309.

25. Wang, Y. F.; Chao, H. R.; Wu, C. H.; Tseng, C. H.; Kuo, Y. T.; Tsou, T. C., A recombinant peroxisome proliferator response element-driven luciferase assay for evaluation of potential environmental obesogens. Biotechnol Lett 2010, 32, (12), 1789-96.

26. Pereira-Fernandes, A.; Demaegdt, H.; Vandermeiren, K.; Hectors, T. L.; Jorens, P. G.; Blust, R.; Vanparys, C., Evaluation of a screening system for obesogenic compounds: screening of endocrine disrupting compounds and evaluation of the PPAR dependency of the effect. PLoS One 2013, 8, (10), e77481.

27. Wei, C.; Wang, X.; Yao, X.; Xi, F.; He, Y.; Xu, Y.; Ma, L.; Chen, X.; Zhao, C.; Du, R.; Pang, W.; Yang, G.; Yu, T. Y., Bifenthrin induces fat deposition by improving fatty acid uptake and inhibiting lipolysis in mice. J Agric Food Chem 2019, 67, (51), 14048-14055.

28. Martinez, M. A.; Blanco, J.; Rovira, J.; Kumar, V.; Domingo, J. L.; Schuhmacher, M., Bisphenol A analogues (BPS and BPF) present a greater obesogenic capacity in 3T3-L1 cell line. Food Chem Toxicol 2020, 140, 111298.

480 29. Choi, S. I.; Lee, J. S.; Lee, S.; Sim, W. S.; Kim, Y. C.; Lee, O. H., Potentilla rugulosa Nakai extract attenuates Bisphenol A-, S- and F-induced ROS production and differentiation of 3T3-L1 preadipocytes in the absence of dexamethasone. Antioxidants (Basel) 2020, 9, (2).

30. Drobna, Z.; Talarovicova, A.; Schrader, H. E.; Fennell, T. R.; Snyder, R. W.; Rissman, E. F., Bisphenol $\mathrm{F}$ has different effects on preadipocytes differentiation and weight gain in adult mice as compared with Bisphenol A and S. Toxicology 2019, 420, 66-72.

486 31. Chamorro-Garcia, R.; Kirchner, S.; Li, X.; Janesick, A.; Casey, S. C.; Chow, C.; 487 Blumberg, B., Bisphenol A diglycidyl ether induces adipogenic differentiation of multipotent stromal stem cells through a peroxisome proliferator-activated receptor gamma-independent mechanism. Environ Health Perspect 2012, 120, (7), 984-9.

32. Helies-Toussaint, C.; Peyre, L.; Costanzo, C.; Chagnon, M. C.; Rahmani, R., Is bisphenol S a safe substitute for bisphenol A in terms of metabolic function? An in vitro study. Toxicol Appl Pharmacol 2014, 280, (2), 224-35.

33. Ariemma, F.; D'Esposito, V.; Liguoro, D.; Oriente, F.; Cabaro, S.; Liotti, A.; Cimmino, I.; Longo, M.; Beguinot, F.; Formisano, P.; Valentino, R., Low-dose Bisphenol-A impairs adipogenesis and generates dysfunctional 3T3-L1 adipocytes. PLoS One 2016, 11, (3), $\mathrm{e} 0150762$. Perspect 2019, 127, (3), 37007.

500 35. Peshdary, V.; Calzadilla, G.; Landry, A.; Sorisky, A.; Atlas, E., Dechlorane Plus increases adipogenesis in 3T3-L1 and human primary preadipocytes independent of peroxisome proliferator-activated receptor gamma transcriptional activity. Int $J$ Obes (Lond) 2019, 43, (3), 545-555. 
36. Feige, J. N.; Gelman, L.; Rossi, D.; Zoete, V.; Metivier, R.; Tudor, C.; Anghel, S. I.;

505 Grosdidier, A.; Lathion, C.; Engelborghs, Y.; Michielin, O.; Wahli, W.; Desvergne, B., The endocrine disruptor monoethyl-hexyl-phthalate is a selective peroxisome proliferator-activated receptor gamma modulator that promotes adipogenesis. J Biol Chem 2007, 282, (26), 1915250866.

509 37. Biserni, M.; Mesnage, R.; Ferro, R.; Wozniak, E.; Xenakis, T.; Mein, C. A.; Antoniou,

510 M. N., Quizalofop-p-Ethyl induces adipogenesis in 3T3-L1 adipocytes. Toxicol Sci 2019, 170,

511 (2), 452-461.

512 38. Mesnage, R.; Biserni, M.; Genkova, D.; Wesolowski, L.; Antoniou, M. N., Evaluation 513 of neonicotinoid insecticides for oestrogenic, thyroidogenic and adipogenic activity reveals 514 imidacloprid causes lipid accumulation. J Appl Toxicol 2018, 38, (12), 1483-1491.

515 39. Smith, A.; Yu, X.; Yin, L., Diazinon exposure activated transcriptional factors 516 CCAAT-enhancer-binding proteins alpha (C/EBPalpha) and peroxisome proliferator-activated 517 receptor gamma (PPARgamma) and induced adipogenesis in 3T3-L1 preadipocytes. Pestic 518 Biochem Physiol 2018, 150, 48-58.

519 40. Temkin, A. M.; Bowers, R. R.; Magaletta, M. E.; Holshouser, S.; Maggi, A.; Ciana, P.; 520 Guillette, L. J.; Bowden, J. A.; Kucklick, J. R.; Baatz, J. E.; Spyropoulos, D. D., Effects of 521 crude oil/dispersant mixture and dispersant components on PPARgamma activity in vitro and 522 in vivo: Identification of Dioctyl Sodium Sulfosuccinate (DOSS; CAS \#577-11-7) as a probable obesogen. Environ Health Perspect 2016, 124, (1), 112-9.

41. Bowers, R. R.; Temkin, A. M.; Guillette, L. J.; Baatz, J. E.; Spyropoulos, D. D., The commonly used nonionic surfactant Span 80 has RXRalpha transactivation activity, which likely increases the obesogenic potential of oil dispersants and food emulsifiers. Gen Comp Endocrinol 2016, 238, 61-68.

42. Cano-Sancho, G.; Smith, A.; La Merrill, M. A., Triphenyl phosphate enhances adipogenic differentiation, glucose uptake and lipolysis via endocrine and noradrenergic mechanisms. Toxicol In Vitro 2017, 40, 280-288.

531 43. Xie, X.; Yu, C.; Ren, Q.; Wen, Q.; Zhao, C.; Tang, Y.; Du, Y., Exposure to HBCD promotes adipogenesis both in vitro and in vivo by interfering with Wnt6 expression. Sci Total Environ 2020, 705, 135917.

44. Tung, E. W. Y.; Ahmed, S.; Peshdary, V.; Atlas, E., Firemaster(R) 550 and its components isopropylated triphenyl phosphate and triphenyl phosphate enhance adipogenesis and transcriptional activity of peroxisome proliferator activated receptor (PPARgamma) on the adipocyte protein 2 (aP2) promoter. PLoS One 2017, 12, (4), e0175855.

538 45. Elmore, S. E.; Cano-Sancho, G.; La Merrill, M. A., Disruption of normal adipocyte 539 development and function by methyl- and propyl- paraben exposure. Toxicol Lett 2020, 334, $540 \quad 27-35$.

541 46. Hao, C.; Cheng, X.; Xia, H.; Ma, X., The endocrine disruptor mono-(2-ethylhexyl) 542 phthalate promotes adipocyte differentiation and induces obesity in mice. Biosci Rep 2012, 32, 543 (6), 619-29. 

brominated flame retardant PBDE 99 promotes adipogenesis via regulating mitotic clonal expansion and PPARgamma expression. Sci Total Environ 2019, 670, 67-77.

547 48. Watkins, A. M.; Wood, C. R.; Lin, M. T.; Abbott, B. D., The effects of perfluorinated chemicals on adipocyte differentiation in vitro. Mol Cell Endocrinol 2015, 400, (C), 90-101.

49. Ma, Y.; Yang, J.; Wan, Y.; Peng, Y.; Ding, S.; Li, Y.; Xu, B.; Chen, X.; Xia, W.; Ke, $\mathrm{Y}$.; Xu, S., Low-level perfluorooctanoic acid enhances $3 \mathrm{~T} 3-\mathrm{L} 1$ preadipocyte differentiation via altering peroxisome proliferator activated receptor gamma expression and its promoter DNA methylation. $J$ Appl Toxicol 2018, 38, (3), 398-407.

50. Neel, B. A.; Brady, M. J.; Sargis, R. M., The endocrine disrupting chemical tolylfluanid alters adipocyte metabolism via glucocorticoid receptor activation. Mol Endocrinol 2013, 27, (3), 394-406.

51. Pereira-Fernandes, A.; Vanparys, C.; Hectors, T. L.; Vergauwen, L.; Knapen, D.; Jorens, P. G.; Blust, R., Unraveling the mode of action of an obesogen: mechanistic analysis of the model obesogen tributyltin in the 3T3-L1 cell line. Mol Cell Endocrinol 2013, 370, (12), 52-64.

560 52. Grun, F.; Watanabe, H.; Zamanian, Z.; Maeda, L.; Arima, K.; Cubacha, R.; Gardiner, D. M.; Kanno, J.; Iguchi, T.; Blumberg, B., Endocrine-disrupting organotin compounds are potent inducers of adipogenesis in vertebrates. Mol Endocrinol 2006, 20, (9), 2141-55.

563 53. Inadera, H.; Shimomura, A., Environmental chemical tributyltin augments adipocyte 564 differentiation. Toxicol Lett 2005, 159, (3), 226-34.

565 54. Kim, S.; Li, A.; Monti, S.; Schlezinger, J. J., Tributyltin induces a transcriptional 566 response without a brite adipocyte signature in adipocyte models. Arch Toxicol 2018, 92, (9), $567 \quad 2859-2874$.

568 55. Li, X.; Ycaza, J.; Blumberg, B., The environmental obesogen tributyltin chloride acts 569 via peroxisome proliferator activated receptor gamma to induce adipogenesis in murine 3T3L1 preadipocytes. J Steroid Biochem Mol Biol 2011, 127, (1-2), 9-15.

56. Regnier, S. M.; El-Hashani, E.; Kamau, W.; Zhang, X.; Massad, N. L.; Sargis, R. M., Tributyltin differentially promotes development of a phenotypically distinct adipocyte. Obesity 2015, 23, (9), 1864-71.

57. $\quad$ Dong, M.; Yuan, P.; Song, Y.; Lei, H.; Chen, G.; Zhu, X.; Wu, F.; Chen, C.; Liu, C.; Shi, Z.; Zhang, L., In vitro effects of Triclocarban on adipogenesis in murine preadipocyte and human hepatocyte. J Hazard Mater 2020, 399, 122829.

58. Li, X.; Pham, H. T.; Janesick, A. S.; Blumberg, B., Triflumizole is an obesogen in mice that acts through peroxisome proliferator activated receptor gamma (PPARgamma). Environ Health Perspect 2012, 120, (12), 1720-6.

580 59. Kim, S.; Rabhi, N.; Blum, B. C.; Hekman, R.; Wynne, K.; Emili, A.; Farmer, S.; 581 Schlezinger, J. J., Triphenyl phosphate is a selective PPARgamma modulator that does not 582 induce brite adipogenesis in vitro and in vivo. Arch Toxicol 2020, 94, (9), 3087-3103. 